Supplement of Earth Syst. Dynam., 8, 719-747, 2017

https://doi.org/10.5194/esd-8-719-2017-supplement

(C) Author(s) 2017. This work is distributed under

the Creative Commons Attribution 3.0 License.

Earth System
Dynamics

(c) (1)

Supplement of

\title{
Comparison of land surface humidity between observations and CMIP5 models
}

\section{Robert J. H. Dunn et al.}

Correspondence to: Robert J. H. Dunn (robert.dunn@metoffice.gov.uk)

The copyright of individual parts of the supplement might differ from the CC BY 3.0 License. 
Supplementary Information

Temperature 

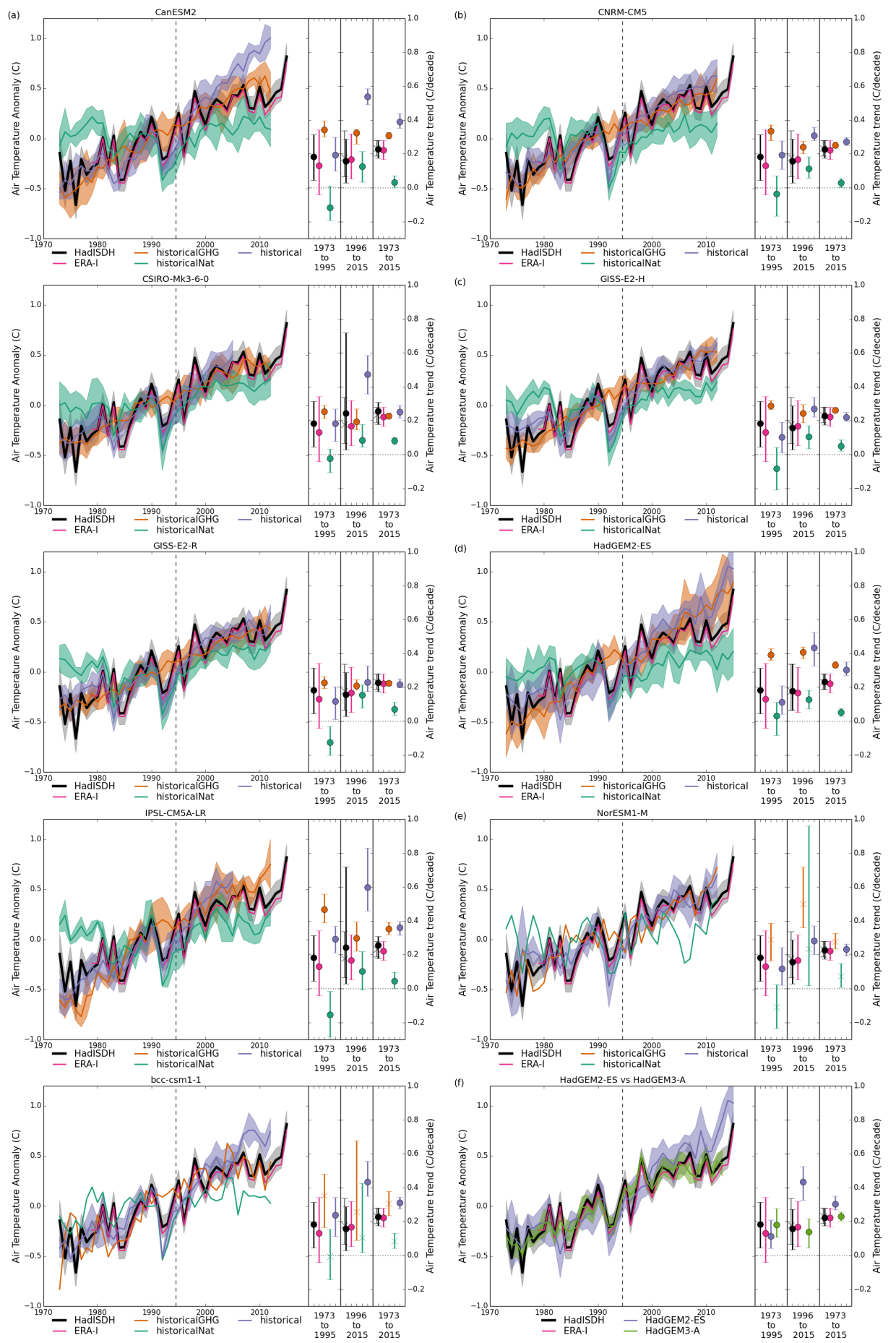

Figure S1. Global annual timeseries for temperature for the nine CMIP5 models and HadGEM3-A, using a climatology period of 19762005. HadISDH is shown by the thick black line, ERA-Interim by the magenta line and the historical, historicalNat and historicalGHG ensemble averages by the purple, green and orange lines respectively. The uncertainty ranges are shown using the coloured shading. The right-hand panels show the values of the linear trends for HadISDH, ERA-Interim and the ensemble averages of all three experiments for the early (1973-1994), late (1995-2015) and full periods. For the late and full period panels, the HadISDH trend is shown matching the temporal coverage of the historical model (circle) and its full coverage (cross). If there is only one ensemble member for the model, then the trend is marked with a cross rather than circle. 

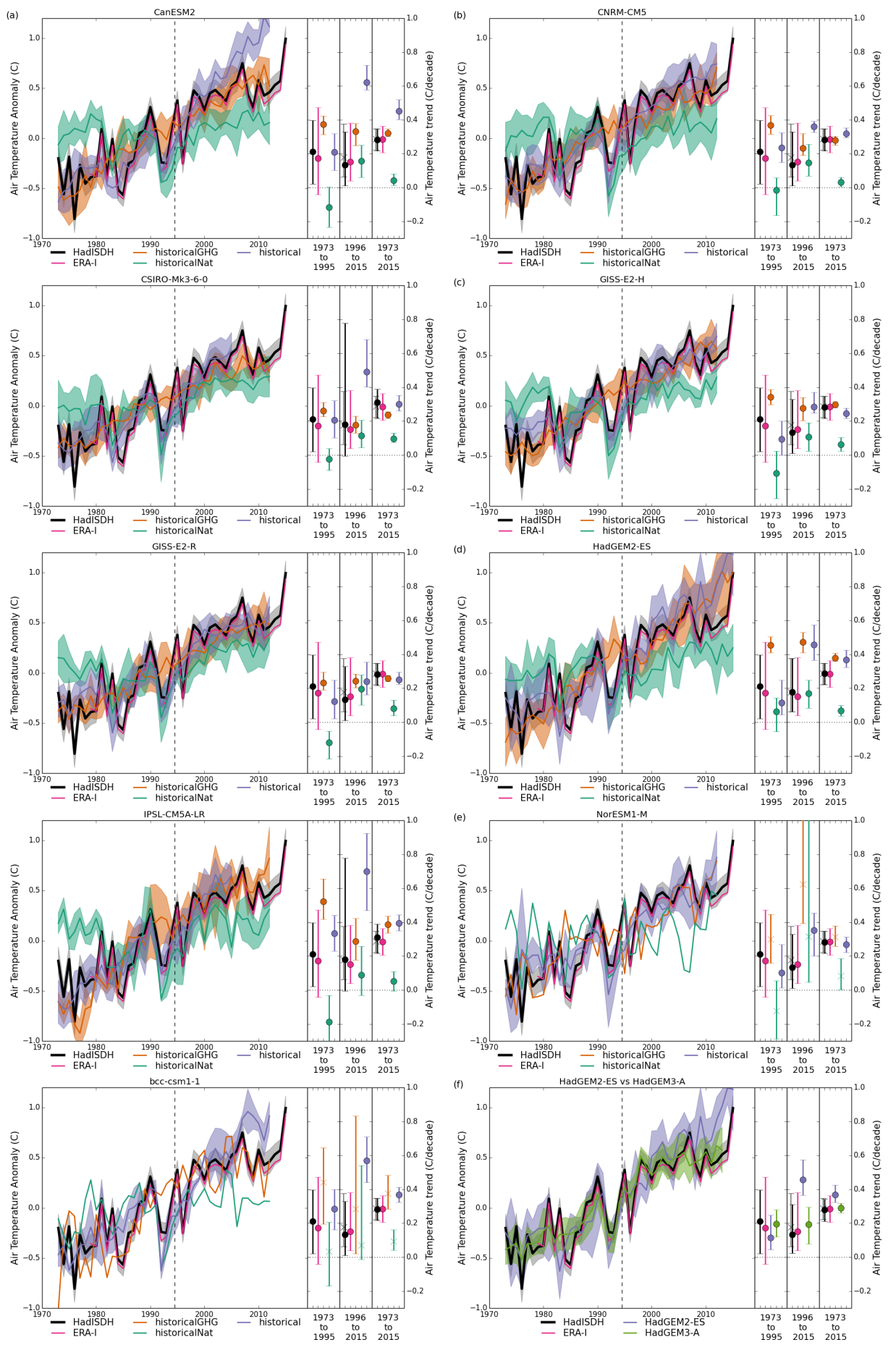

Figure S2. Northern Hemisphere annual timeseries for temperature for the nine CMIP5 models and HadGEM3-A, using a climatology period of 1976-2005. HadISDH is shown by the thick black line, ERA-Interim by the magenta line and the historical, historicalNat and historicalGHG ensemble averages by the purple, green and orange lines respectively. The uncertainty ranges are shown using the coloured shading. The right-hand panels show the values of the linear trends for HadISDH, ERA-Interim and the ensemble averages of all three experiments for the early (1973-1994), late (1995-2015) and full periods. For the late and full period panels, the HadISDH trend is shown matching the temporal coverage of the historical model (circle) and its full coverage (cross). If there is only one ensemble member for the model, then the trend is marked with a cross rather than circle. 

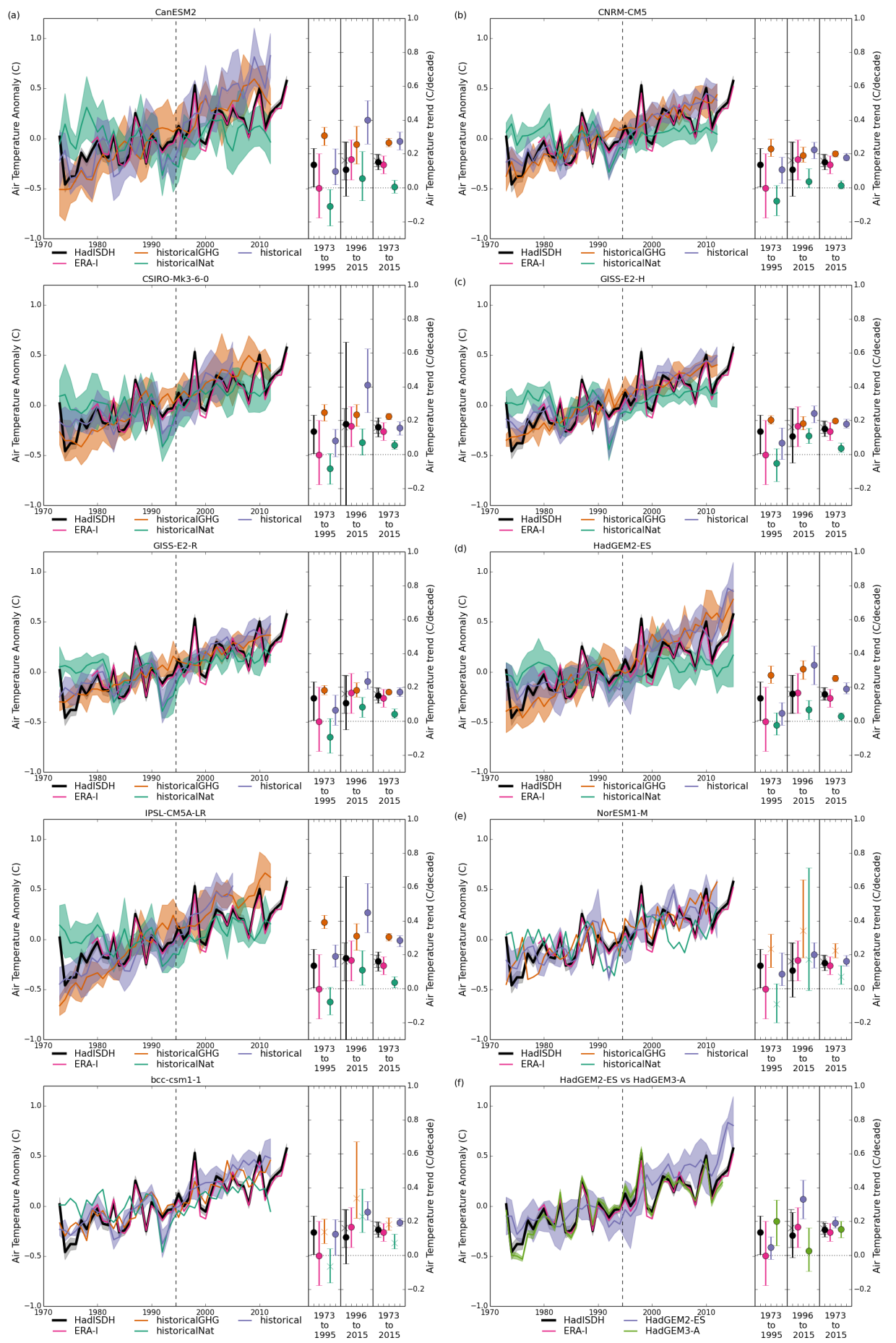

Figure S3. Tropical annual timeseries for temperature for the nine CMIP5 models and HadGEM3-A, using a climatology period of 19762005. HadISDH is shown by the thick black line, ERA-Interim by the magenta line and the historical, historicalNat and historicalGHG ensemble averages by the purple, green and orange lines respectively. The uncertainty ranges are shown using the coloured shading. The right-hand panels show the values of the linear trends for HadISDH, ERA-Interim and the ensemble averages of all three experiments for the early (1973-1994), late (1995-2015) and full periods. For the late and full period panels, the HadISDH trend is shown matching the temporal coverage of the historical model (circle) and its full coverage (cross). If there is only one ensemble member for the model, then the trend is marked with a cross rather than circle. 

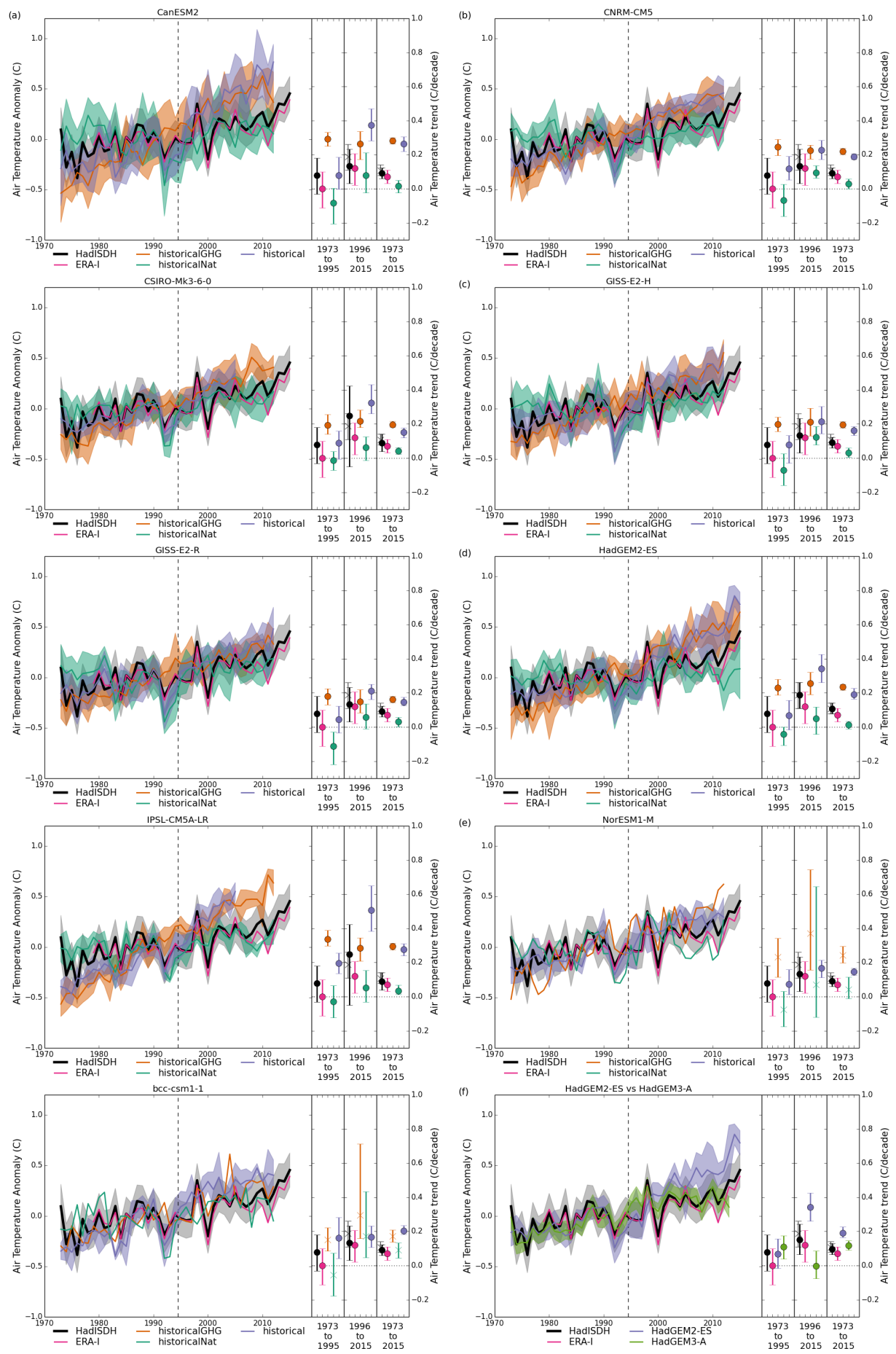

Figure S4. Southern Hemisphere annual timeseries for temperature for the nine CMIP5 models and HadGEM3-A, using a climatology period of 1976-2005. HadISDH is shown by the thick black line, ERA-Interim by the magenta line and the historical, historicalNat and historicalGHG ensemble averages by the purple, green and orange lines respectively. The uncertainty ranges are shown using the coloured shading. The right-hand panels show the values of the linear trends for HadISDH, ERA-Interim and the ensemble averages of all three experiments for the early (1973-1994), late (1995-2015) and full periods. For the late and full period panels, the HadISDH trend is shown matching the temporal coverage of the historical model (circle) and its full coverage (cross). If there is only one ensemble member for the model, then the trend is marked with a cross rather than circle. 

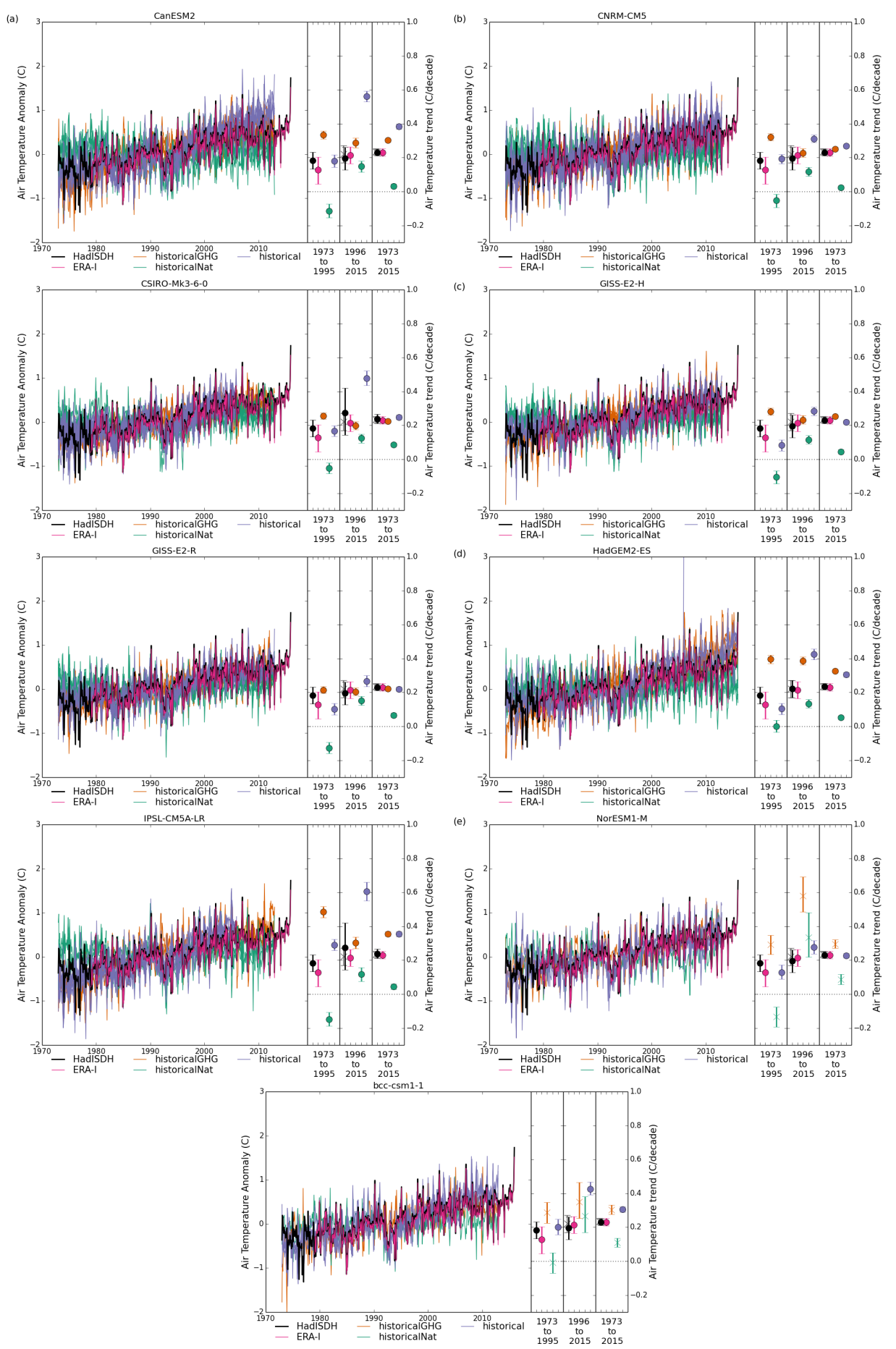

Figure S5. Global monthly timeseries for temperature for the nine CMIP5 models and HadGEM3-A, using a climatology period of 19762005. HadISDH is shown by the thick black line, ERA-Interim by the magenta line and the historical, historicalNat and historicalGHG ensemble members by the purple, green and orange lines respectively. The right-hand panels show the values of the linear trends for HadISDH, ERA-Interim and the ensemble averages of all three experiments for the early (1973-1994), late (1995-2015) and full periods. For the late and full period panels, the HadISDH trend is shown matching the temporal coverage of the historical model (circle) and its full coverage (cross). If there is only one ensemble member for the model, then the trend is marked with a cross rather than circle. 

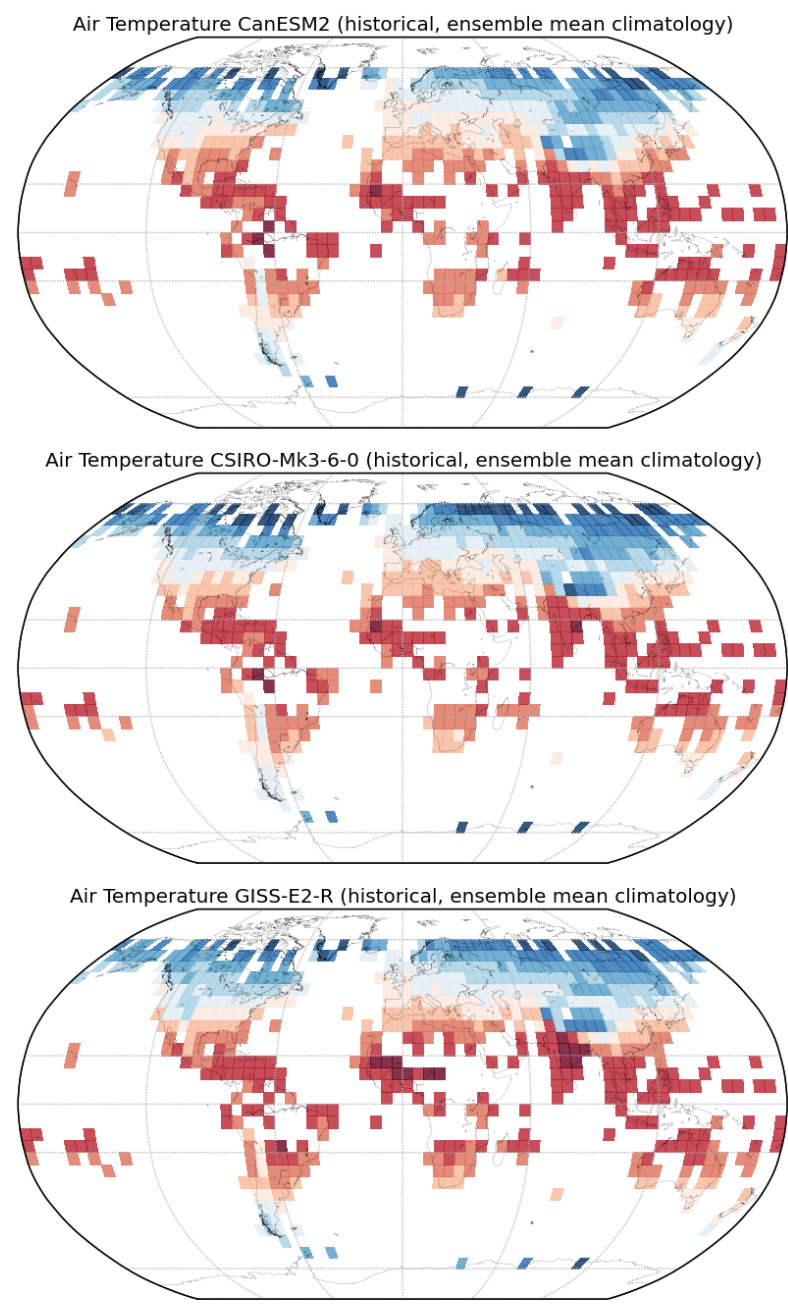

Air Temperature IPSL-CM5A-LR (historical, ensemble mean climatology)
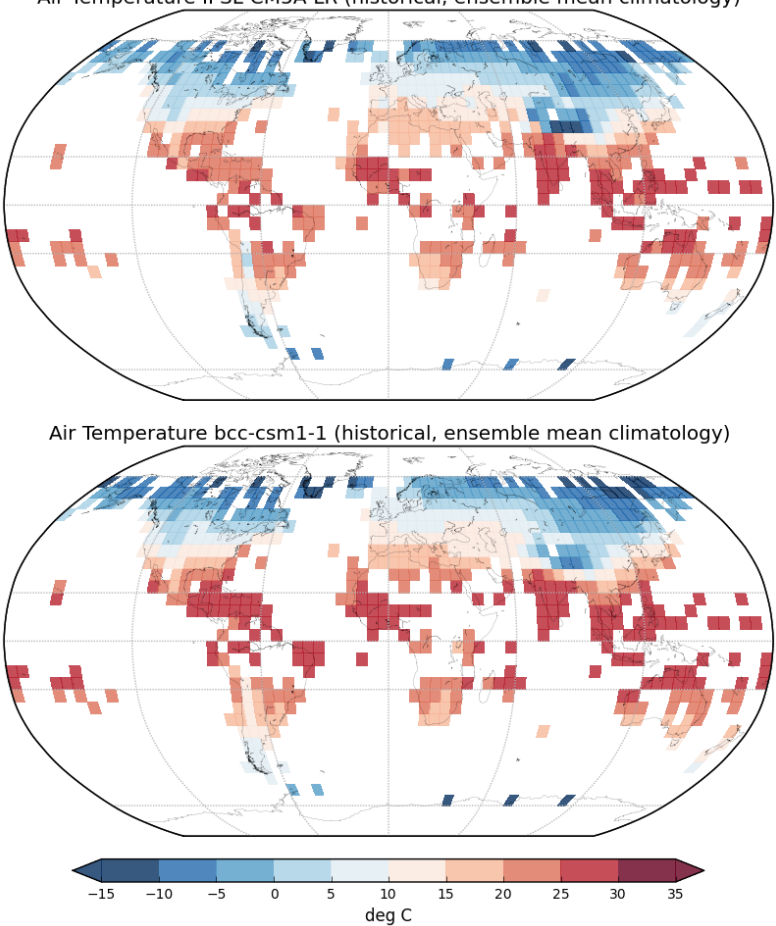

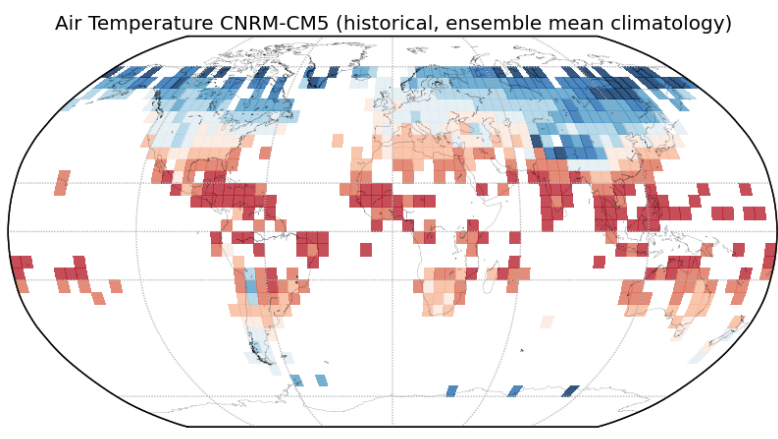

Air Temperature GISS-E2-H (historical, ensemble mean climatology)
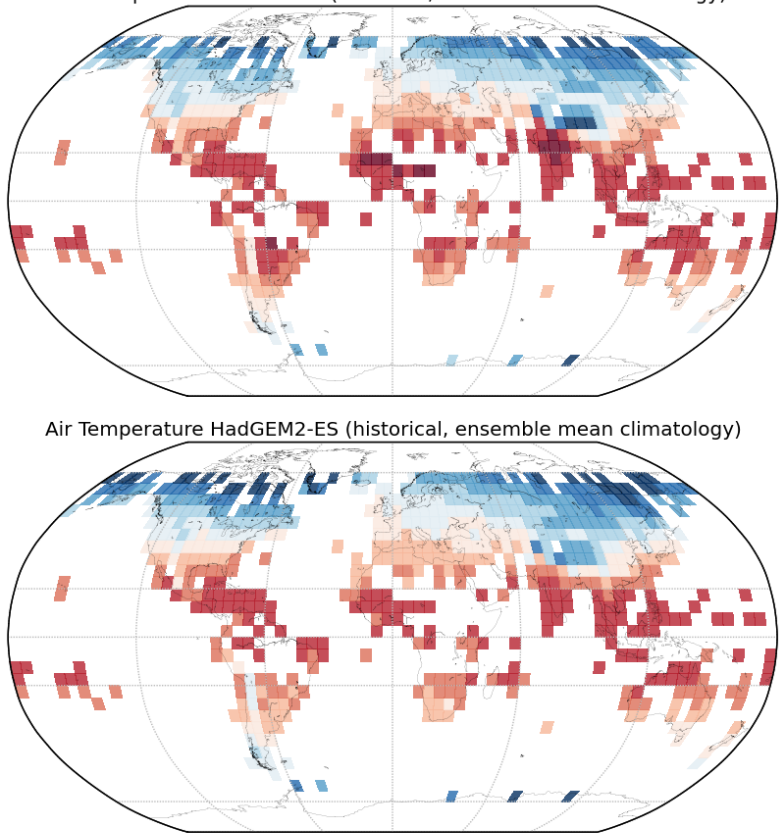

Air Temperature NorESM1-M (historical, ensemble mean climatology)
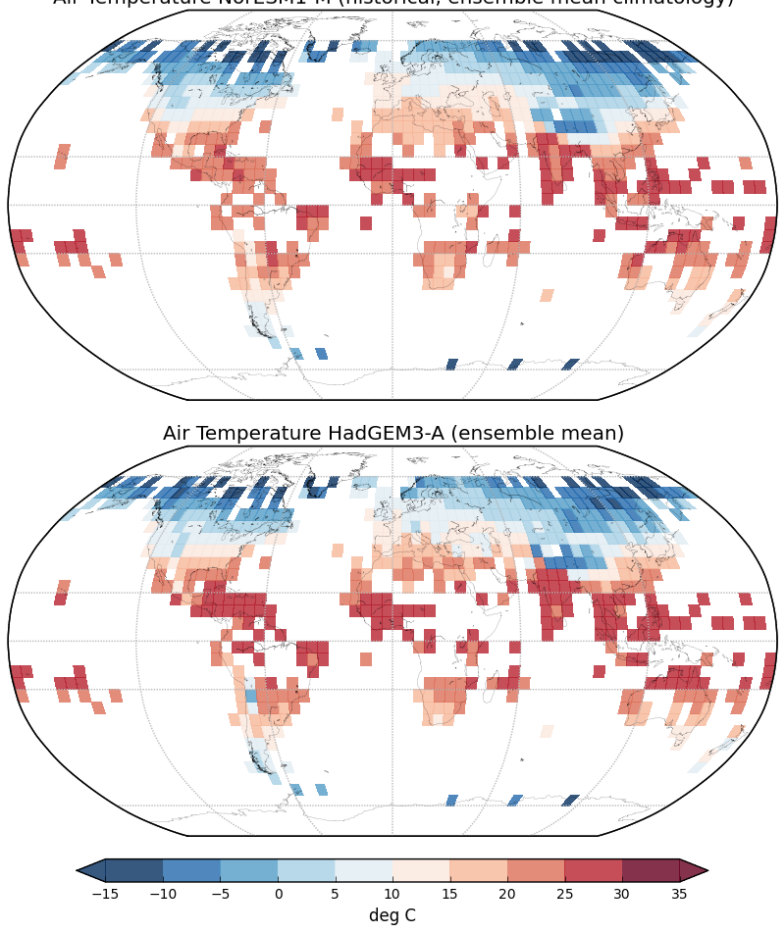

Figure S6. Temperature climatologies of the CMIP5 models at the gridbox scale. All climatologies have been calculated over the 1981 to 2010 period using identical spatio-temporal coverage to HadISDH. 

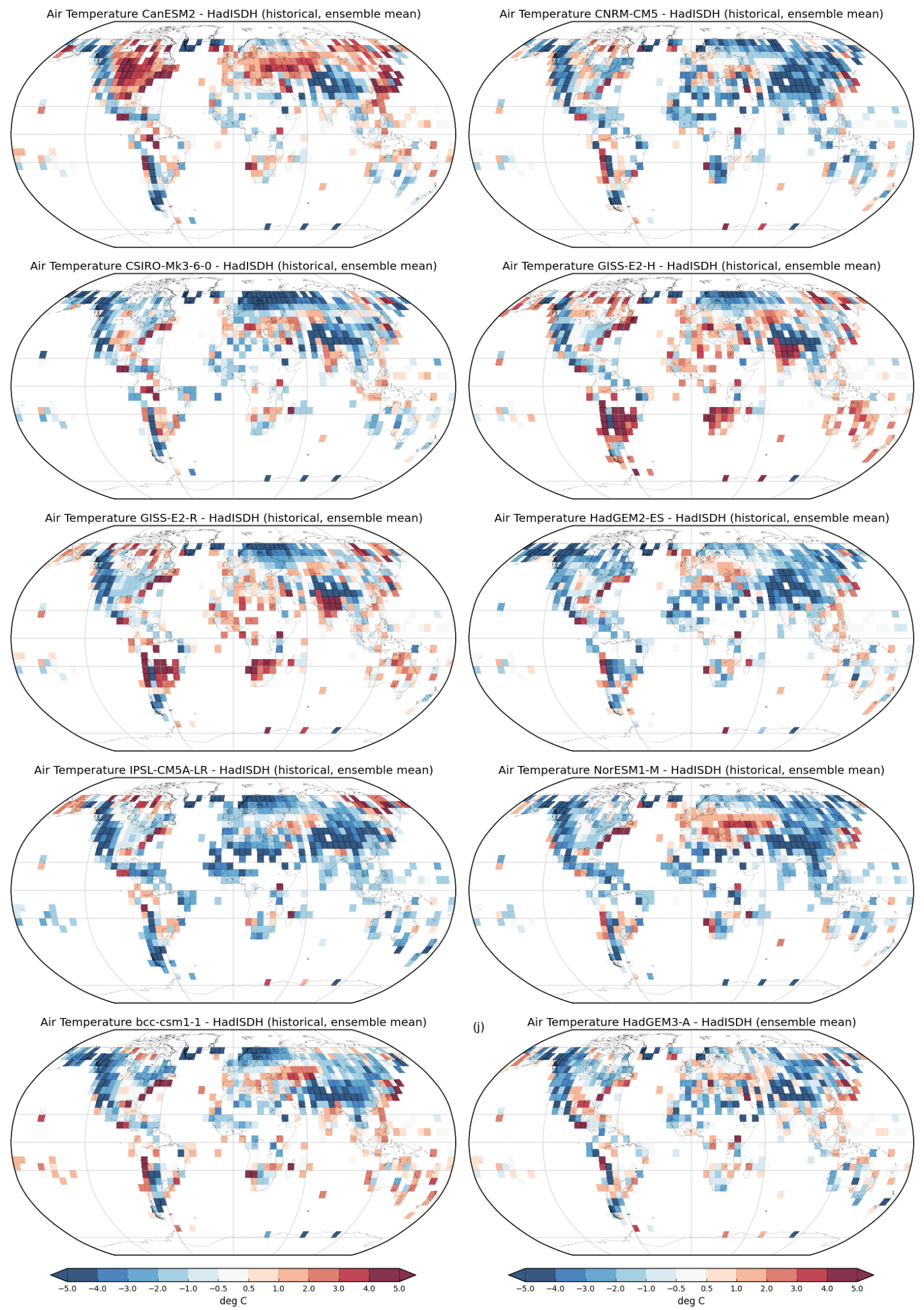

Figure S7. Climatological differences between models and observations at the gridbox scale for temperature. All climatologies have been calculated over the 1981 to 2010 period using identical spatio-temporal coverage to HadISDH. 

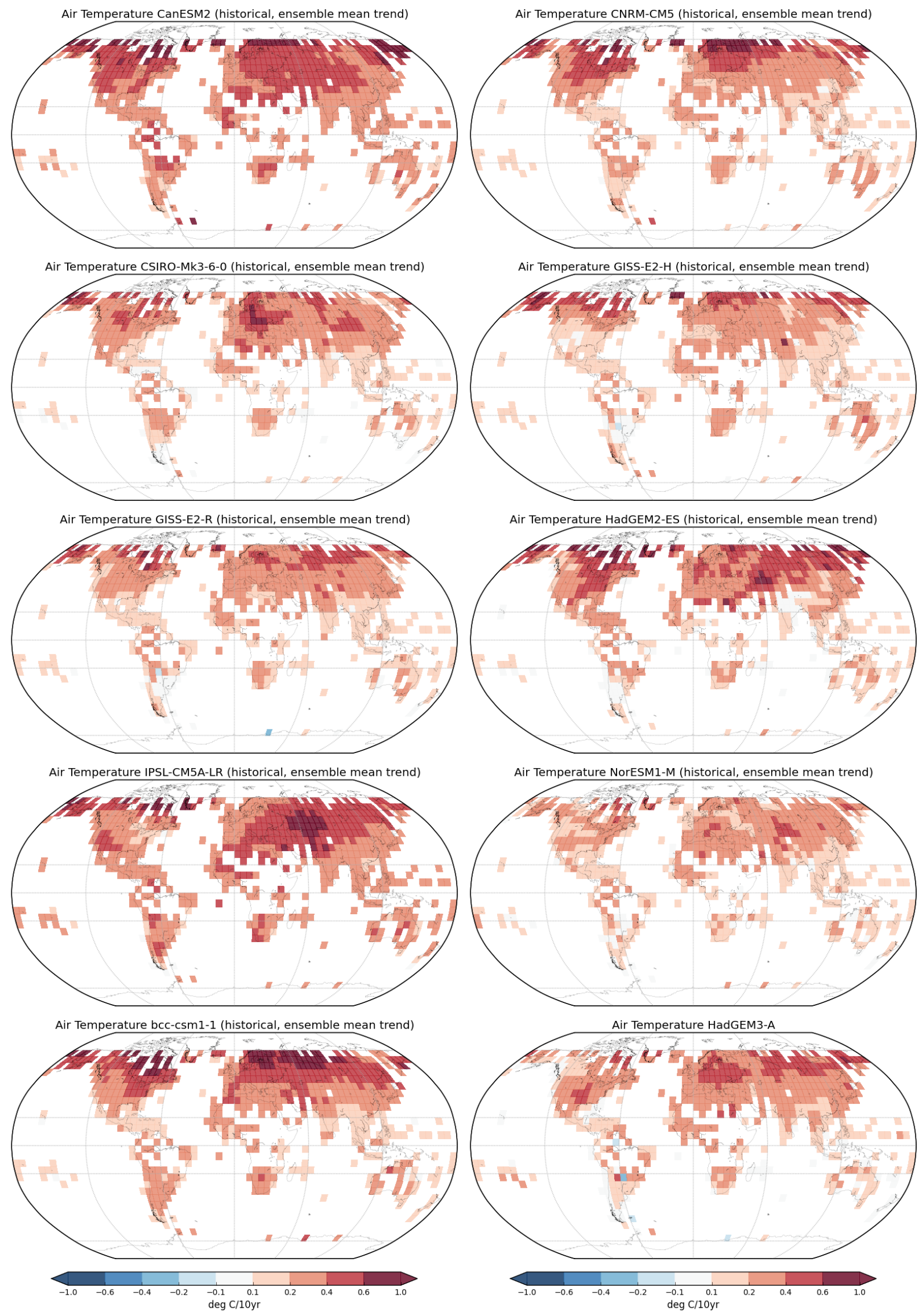

Figure S8. Linear trends of the CMIP5 models at the gridbox scale for temperature. All trends have been calculated using the median of pairwise slopes method, with identical spatio-temporal coverage to HadISDH. 


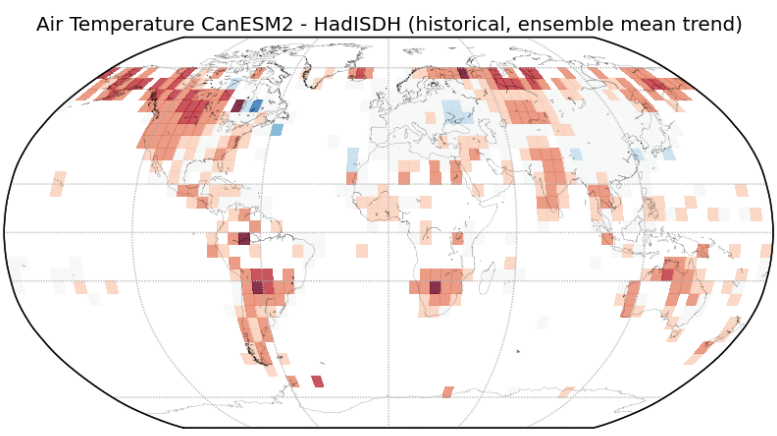

Air Temperature CSIRO-Mk3-6-0 - HadISDH (historical, ensemble mean trend)

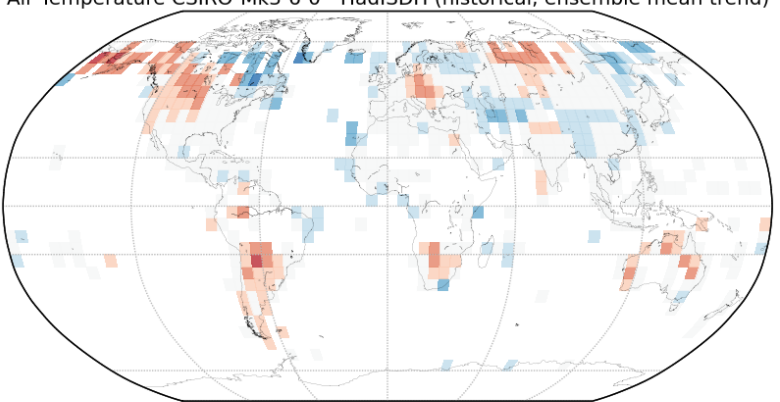

Air Temperature GISS-E2-R - HadISDH (historical, ensemble mean trend)

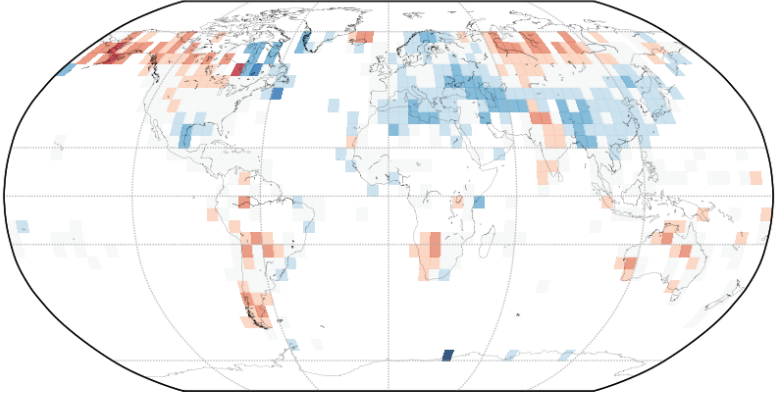

Air Temperature IPSL-CM5A-LR - HadISDH (historical, ensemble mean trend)

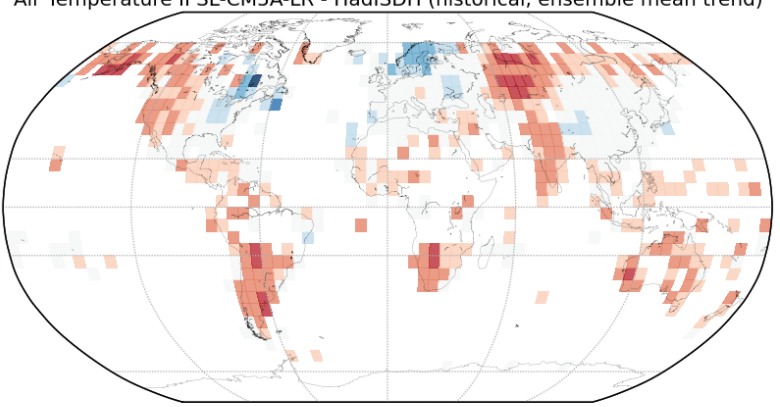

Air Temperature bcc-csm1-1 - HadISDH (historical, ensemble mean trend)

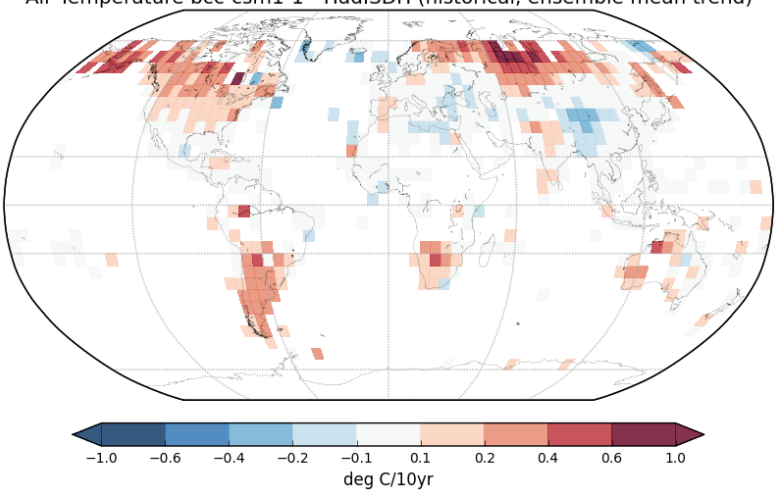

Air Temperature CNRM-CM5 - HadISDH (historical, ensemble mean trend)

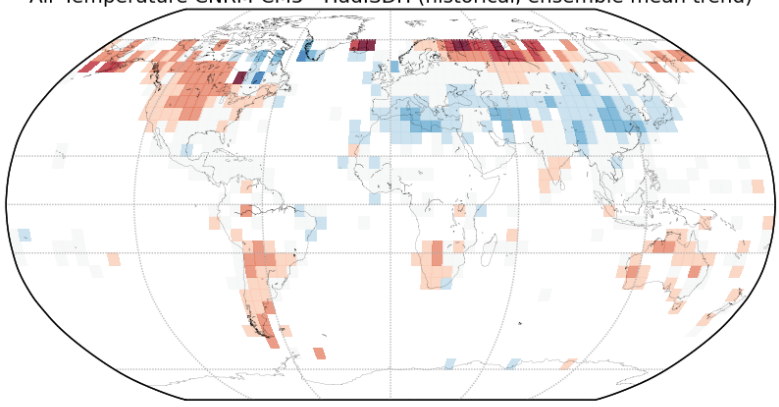

Air Temperature GISS-E2-H - HadISDH (historical, ensemble mean trend)

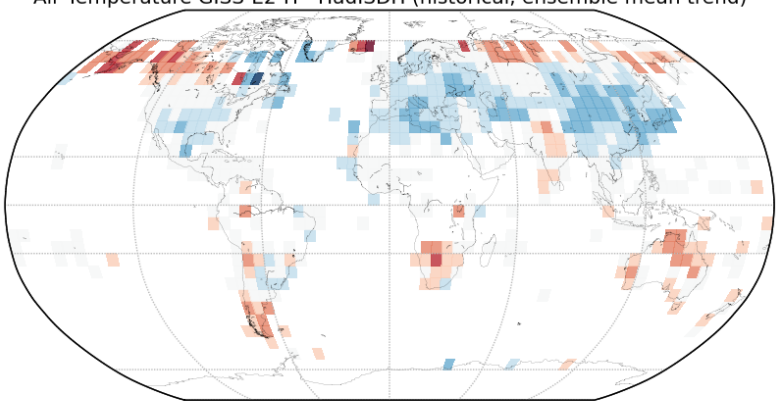

Air Temperature HadGEM2-ES - HadISDH (historical, ensemble mean trend)

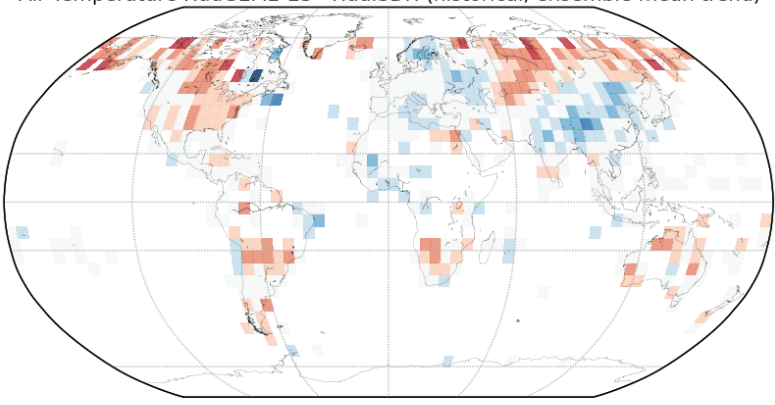

Air Temperature NorESM1-M - HadISDH (historical, ensemble mean trend)
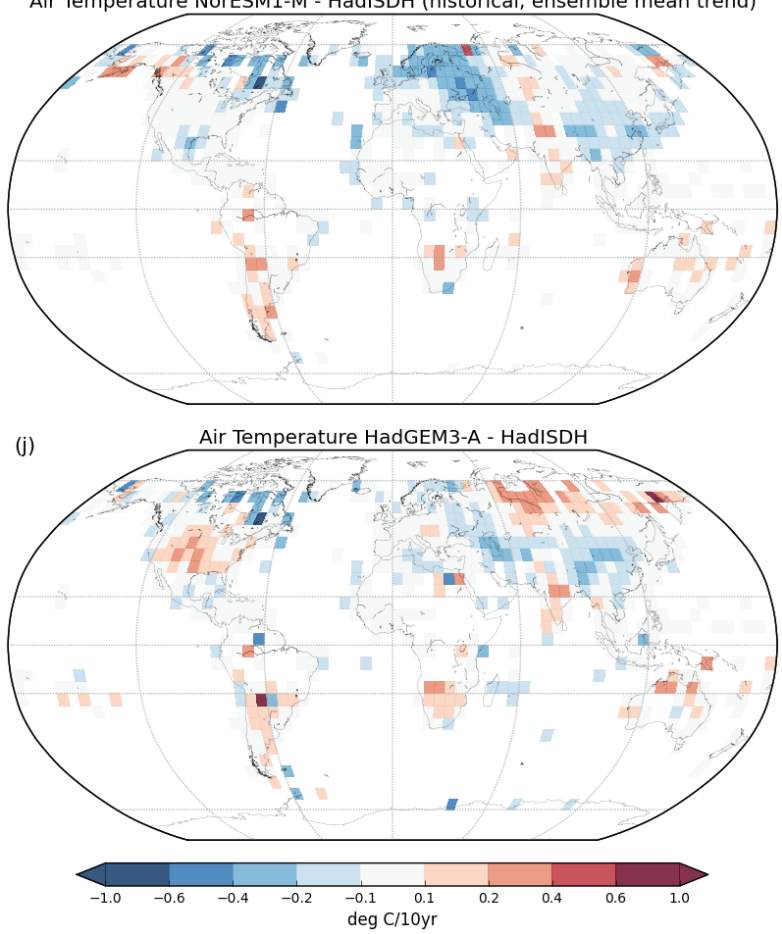

Figure S9. Linear trend differences of the CMIP5 models from the observations at the gridbox scale for temperature. All trends have been calculated using the median of pairwise slopes method, with identical spatio-temporal coverage to HadISDH. 
Relative Humidity 

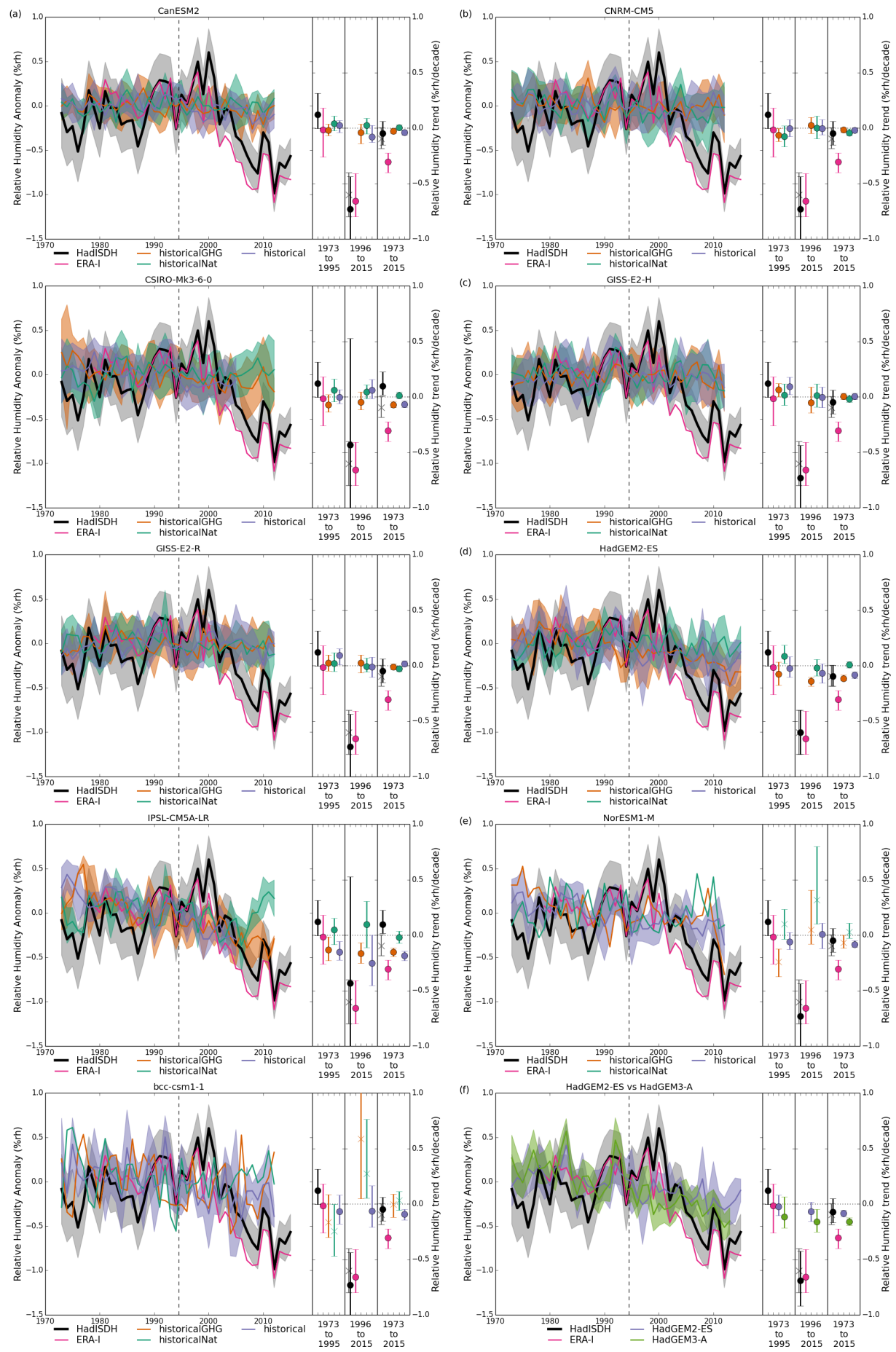

Figure S10. Global annual timeseries for relative humidity for the nine CMIP5 models and HadGEM3-A, using a climatology period of 1976-2005. HadISDH is shown by the thick black line, ERA-Interim by the magenta line and the historical, historicalNat and historicalGHG ensemble averages by the purple, green and orange lines respectively. The uncertainty ranges are shown using the coloured shading. The right-hand panels show the values of the linear trends for HadISDH, ERA-Interim and the ensemble averages of all three experiments for the early (1973-1994), late (1995-2015) and full periods. For the late and full period panels, the HadISDH trend is shown matching the temporal coverage of the historical model (circle) and its full coverage (cross). If there is only one ensemble member for the model, then the trend is marked with a cross rather than circle. 

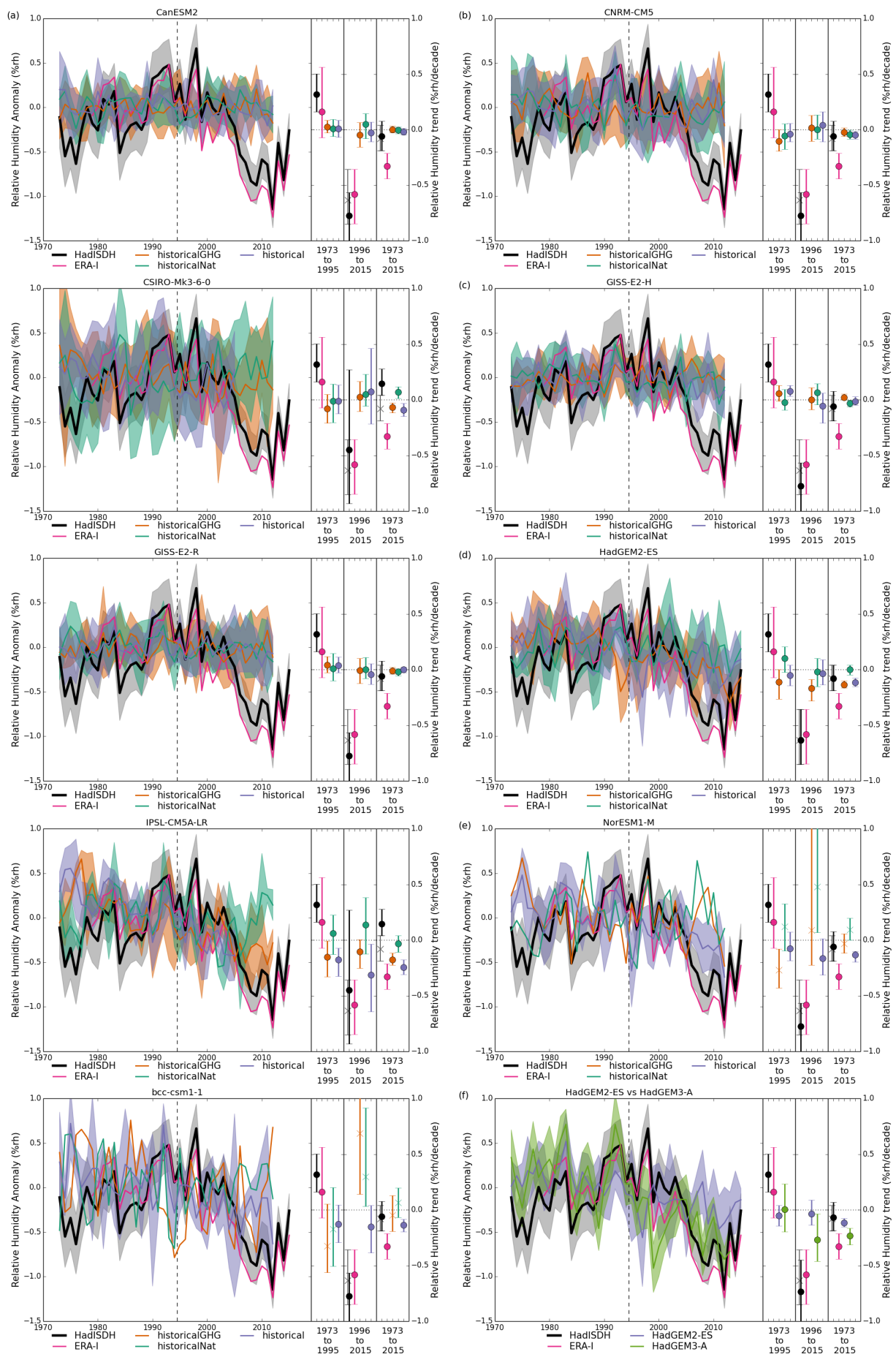

Figure S11. Northern Hemisphere annual timeseries for relative humidity for the nine CMIP5 models and HadGEM3-A, using a climatology period of 1976-2005. HadISDH is shown by the thick black line, ERA-Interim by the magenta line and the historical, historicalNat and historicalGHG ensemble averages by the purple, green and orange lines respectively. The uncertainty ranges are shown using the coloured shading. The right-hand panels show the values of the linear trends for HadISDH, ERA-Interim and the ensemble averages of all three experiments for the early (1973-1994), late (1995-2015) and full periods. For the late and full period panels, the HadISDH trend is shown matching the temporal coverage of the historical model (circle) and its full coverage (cross). If there is only one ensemble member for the model, then the trend is marked with a cross rather than circle. 

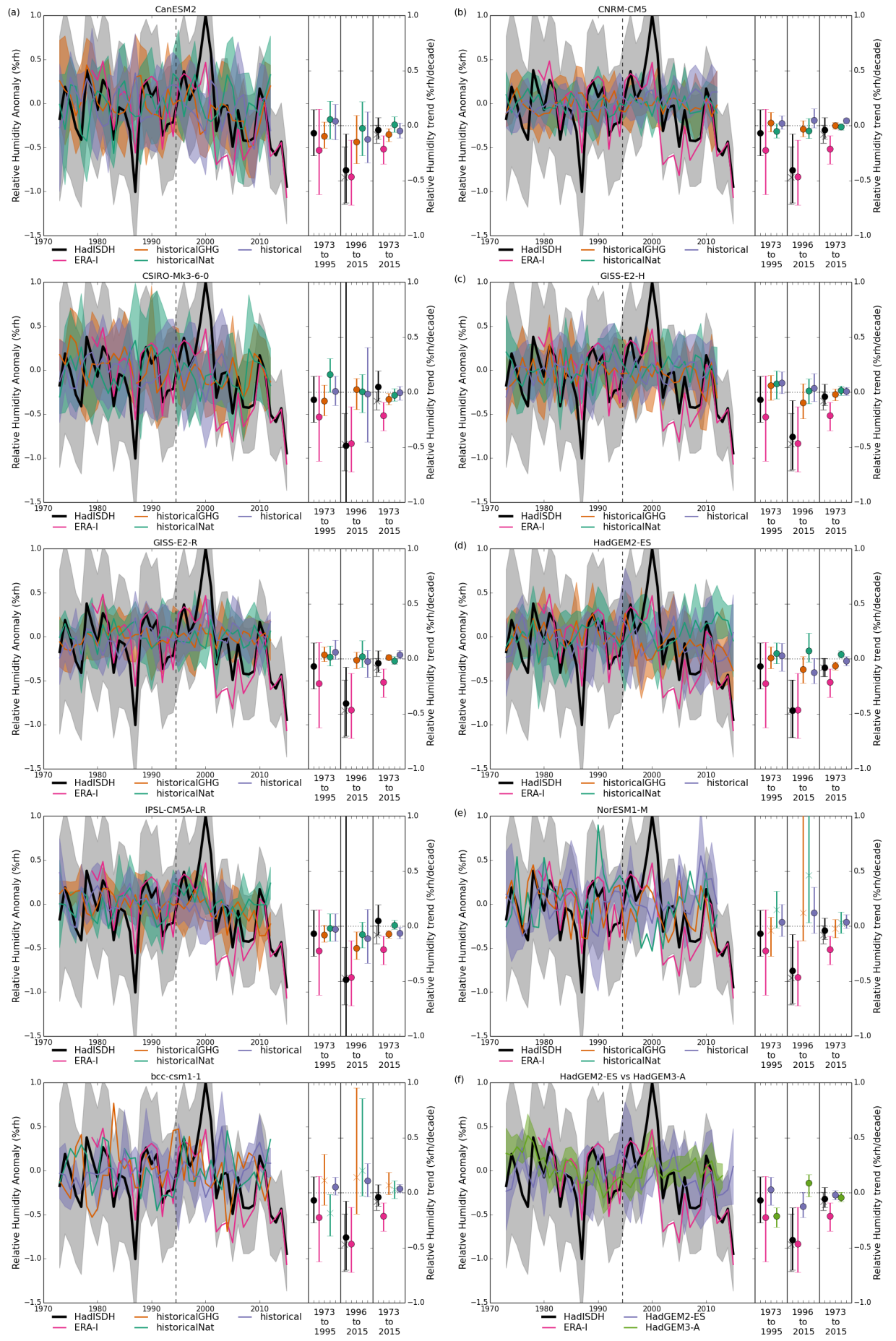

Figure S12. Tropical annual timeseries for relative humidity for the nine CMIP5 models and HadGEM3-A, using a climatology period of 1976-2005. HadISDH is shown by the thick black line, ERA-Interim by the magenta line and the historical, historicalNat and historicalGHG ensemble averages by the purple, green and orange lines respectively. The uncertainty ranges are shown using the coloured shading. The right-hand panels show the values of the linear trends for HadISDH, ERA-Interim and the ensemble averages of all three experiments for the early (1973-1994), late (1995-2015) and full periods. For the late and full period panels, the HadISDH trend is shown matching the temporal coverage of the historical model (circle) and its full coverage (cross). If there is only one ensemble member for the model, then the trend is marked with a cross rather than circle. 

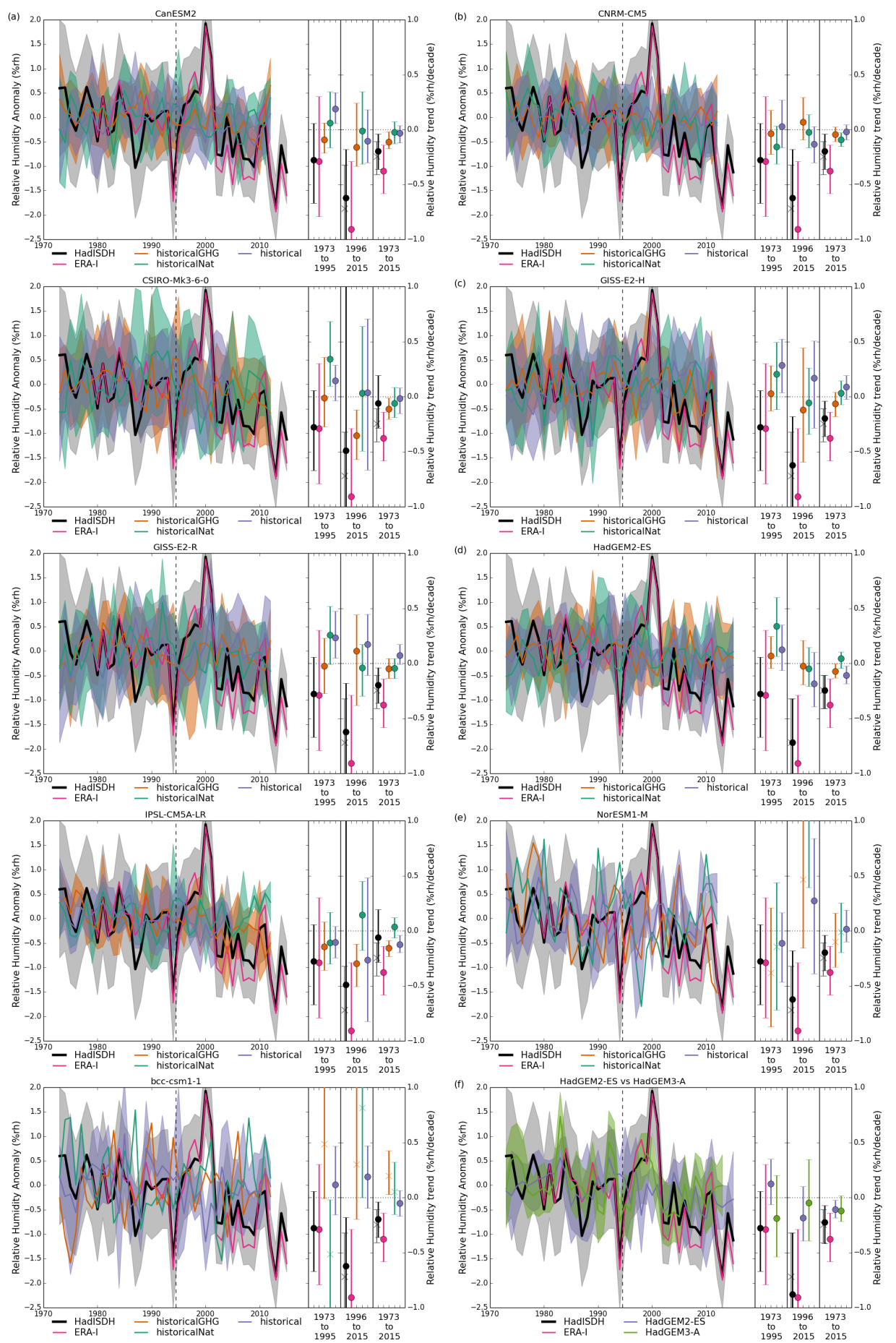

Figure S13. Southern Hemisphere annual timeseries for relative humidity for the nine CMIP5 models and HadGEM3-A, using a climatology period of 1976-2005. HadISDH is shown by the thick black line, ERA-Interim by the magenta line and the historical, historicalNat and historicalGHG ensemble averages by the purple, green and orange lines respectively. The uncertainty ranges are shown using the coloured shading. The right-hand panels show the values of the linear trends for HadISDH, ERA-Interim and the ensemble averages of all three experiments for the early (1973-1994), late (1995-2015) and full periods. For the late and full period panels, the HadISDH trend is shown matching the temporal coverage of the historical model (circle) and its full coverage (cross). If there is only one ensemble member for the model, then the trend is marked with a cross rather than circle. 

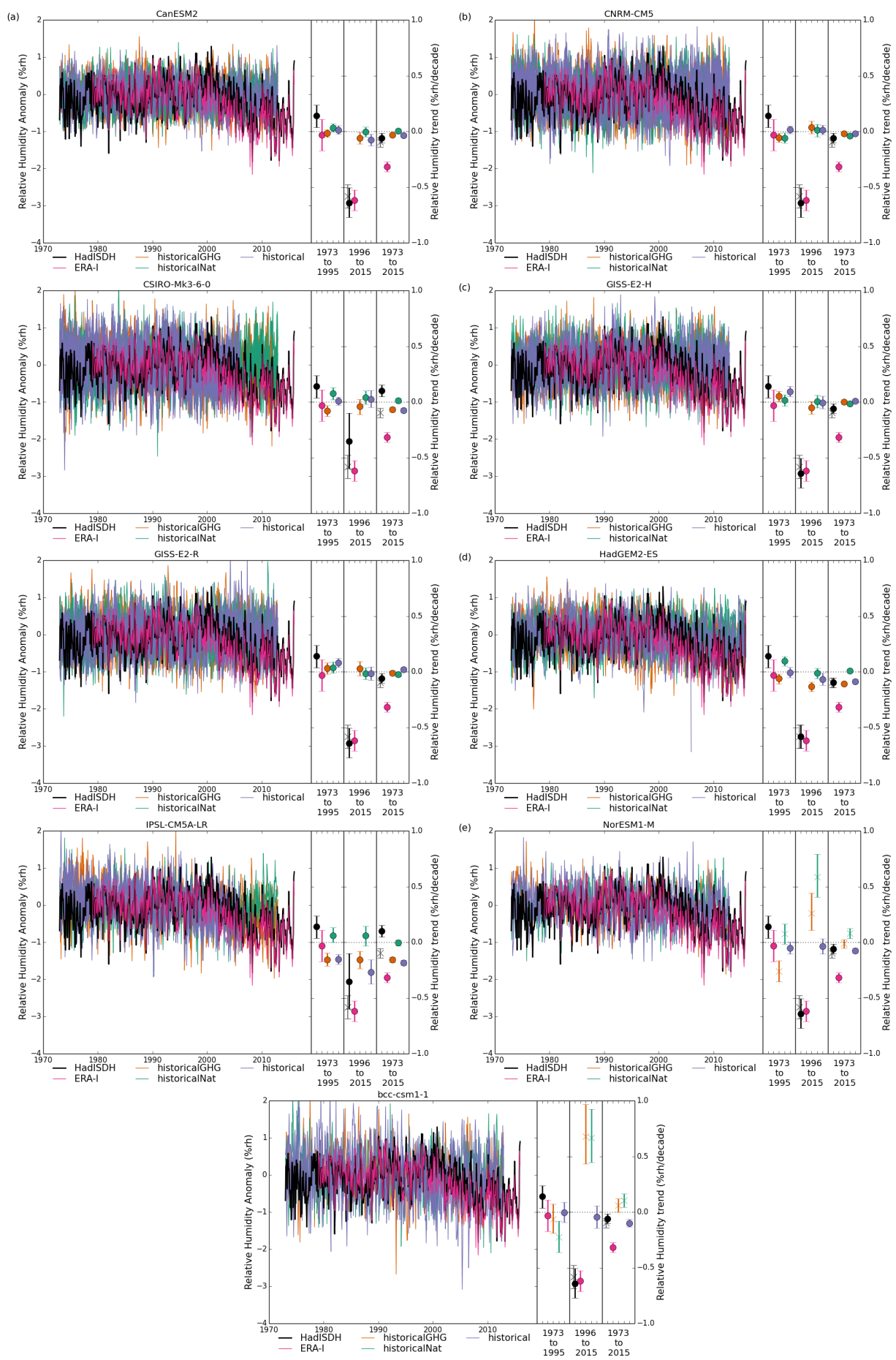

Figure S14. Global annual timeseries for relative humidity for the nine CMIP5 models and HadGEM3-A, using a climatology period of 1976-2005. HadISDH is shown by the thick black line, ERA-Interim by the magenta line and the historical, historicalNat and historicalGHG ensemble members by the purple, green and orange lines respectively. The right-hand panels show the values of the linear trends for HadISDH, ERA-Interim and the ensemble averages of all three experiments for the early (1973-1994), late (1995-2015) and full periods. For the late and full period panels, the HadISDH trend is shown matching the temporal coverage of the historical model (circle) and its full coverage (cross). If there is only one ensemble member for the model, then the trend is marked with a cross rather than circle. 


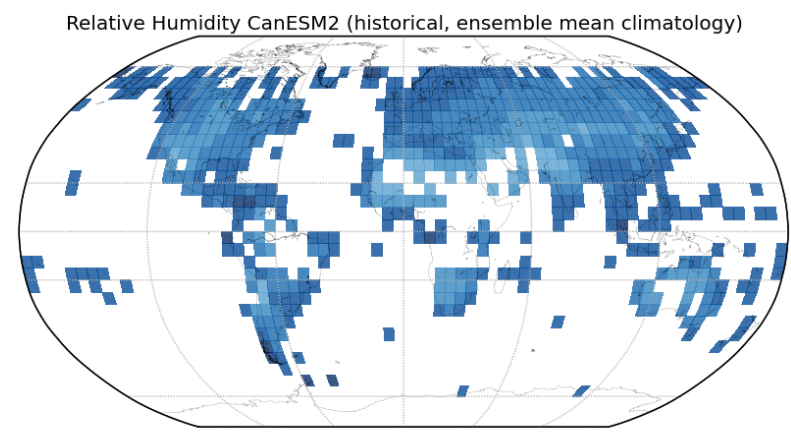

Relative Humidity CSIRO-Mk3-6-0 (historical, ensemble mean climatology)

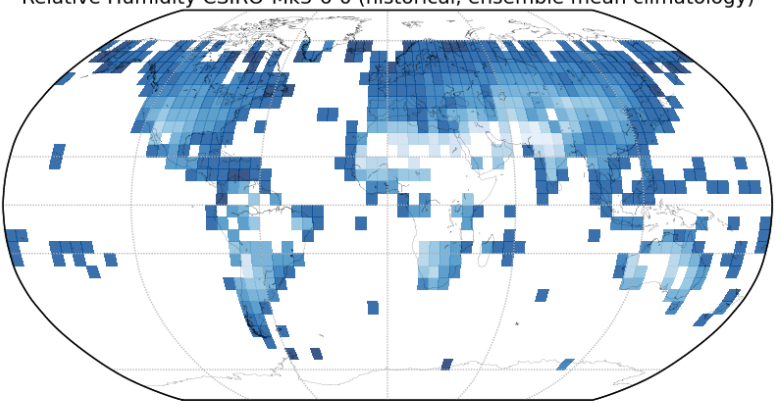

Relative Humidity GISS-E2-R (historical, ensemble mean climatology)

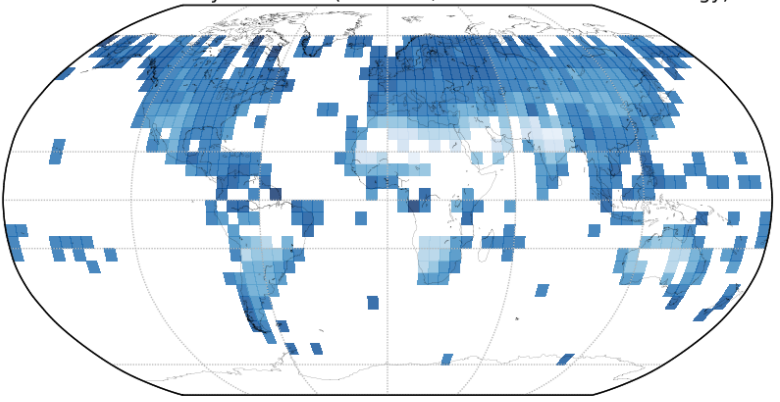

Relative Humidity IPSL-CM5A-LR (historical, ensemble mean climatology)
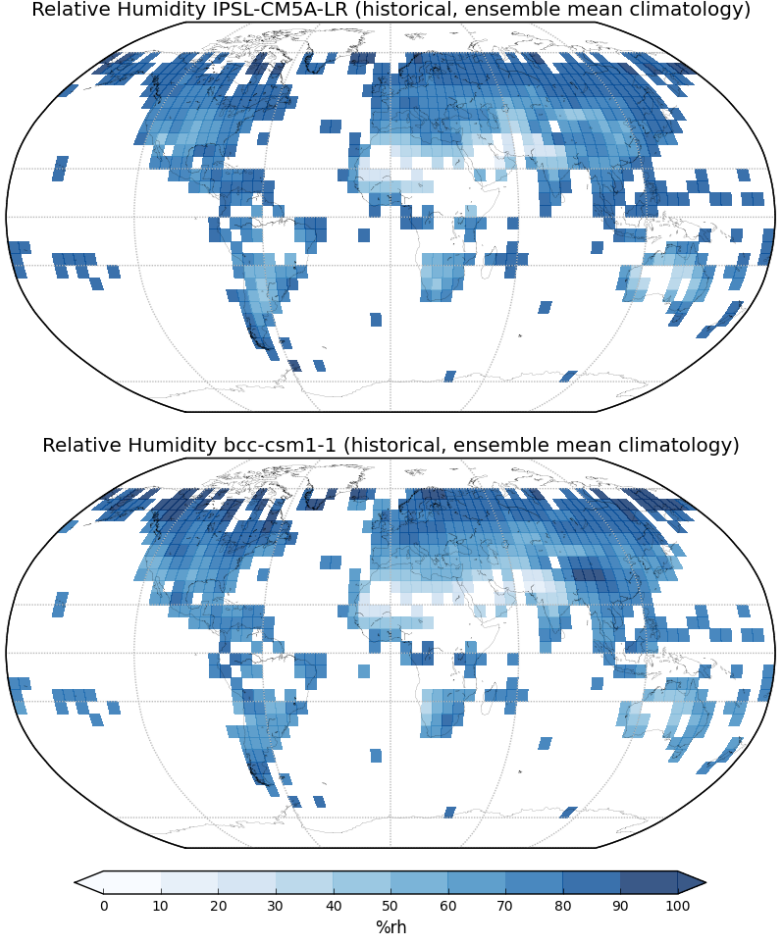

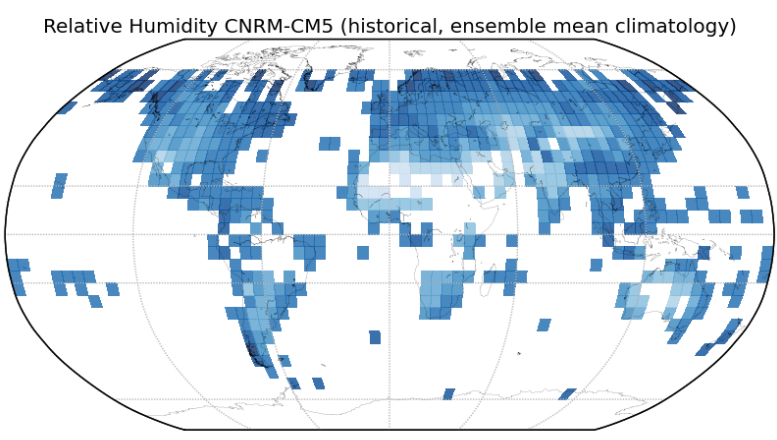

Relative Humidity GISS-E2-H (historical, ensemble mean climatology)

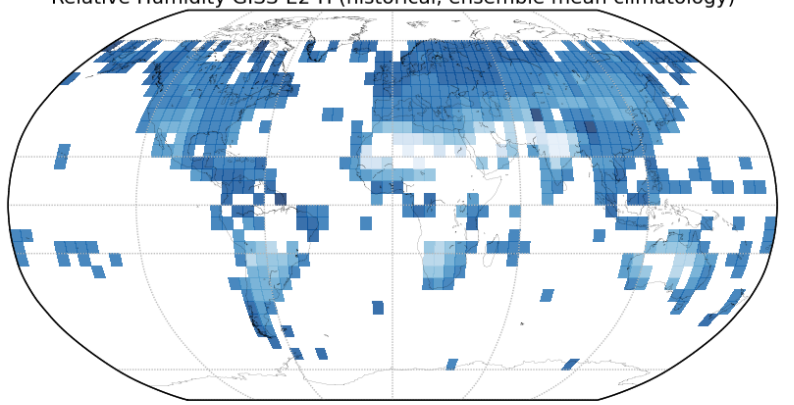

Relative Humidity HadGEM2-ES (historical, ensemble mean climatology)

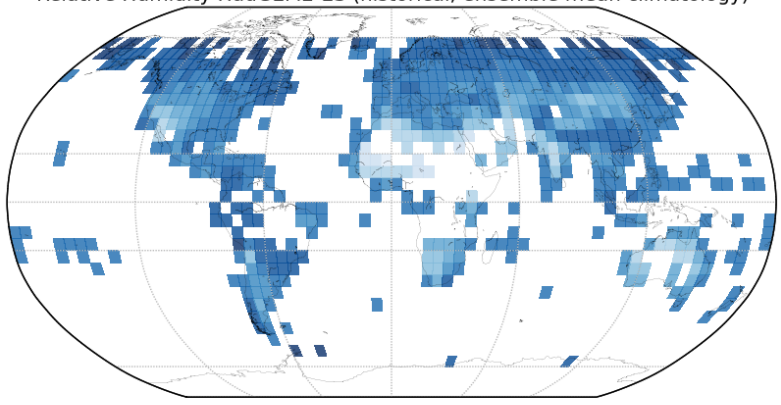

Relative Humidity NorESM1-M (historical, ensemble mean climatology)
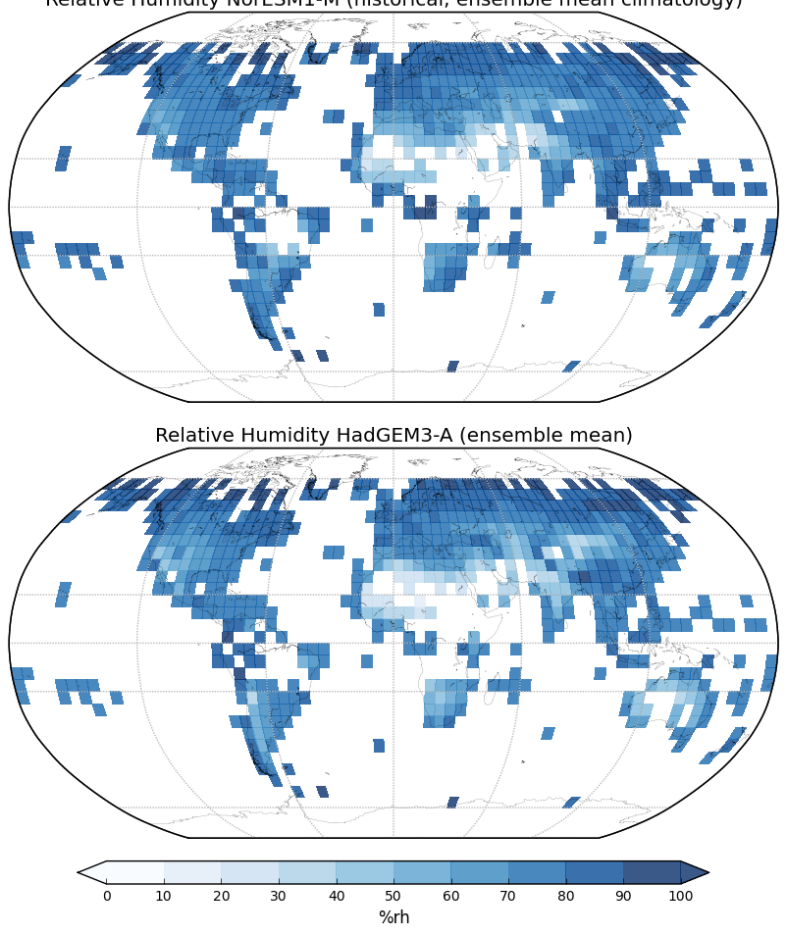

Figure S15. Relative humidity climatologies of the CMIP5 models at the gridbox scale. All climatologies have been calculated over the 1981 to 2010 period using identical spatio-temporal coverage to HadISDH. 


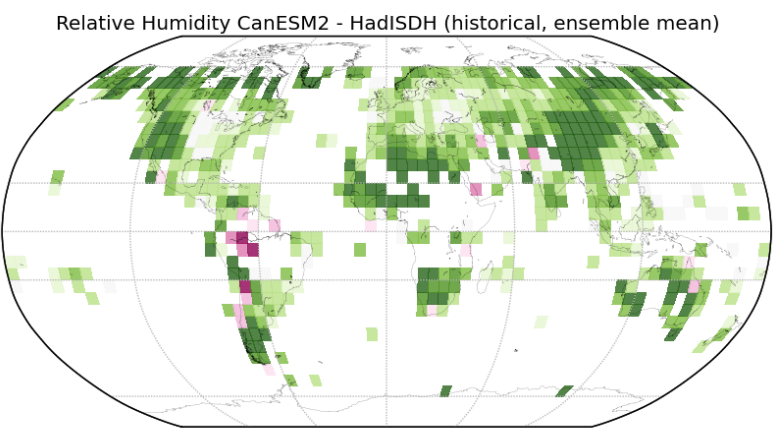

Relative Humidity CSIRO-Mk3-6-0 - HadISDH (historical, ensemble mean)
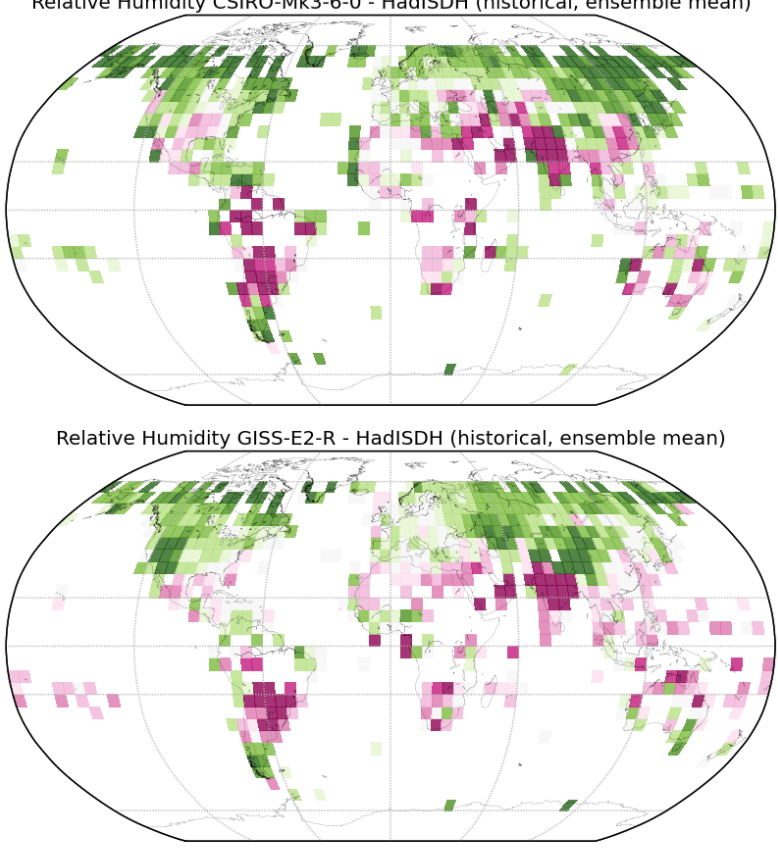

Relative Humidity IPSL-CM5A-LR - HadISDH (historical, ensemble mean)
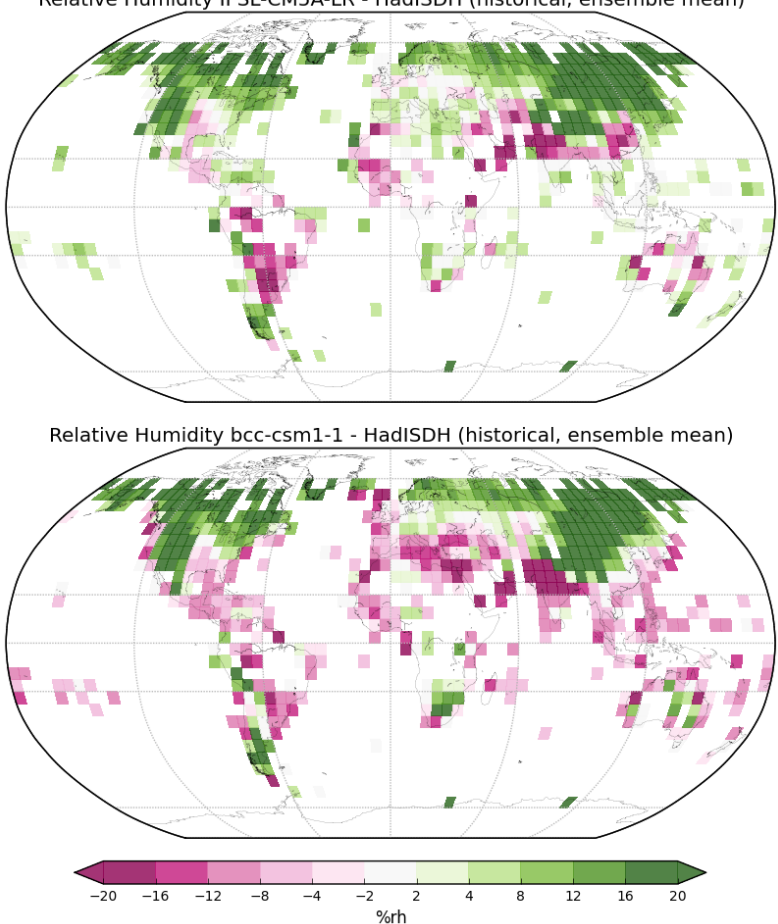

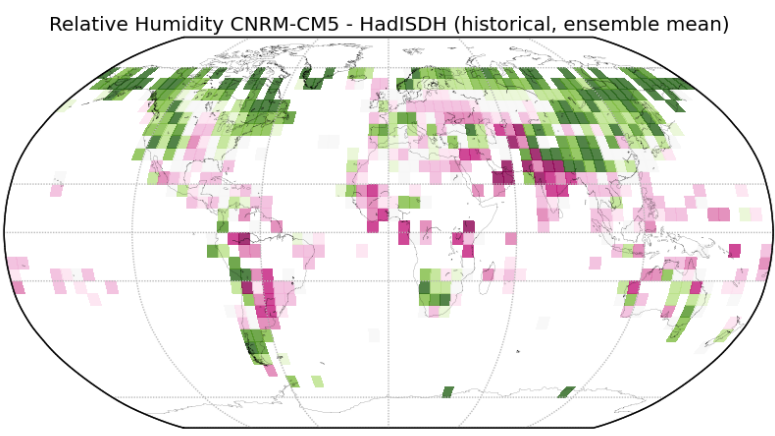

Relative Humidity GISS-E2-H - HadISDH (historical, ensemble mean)
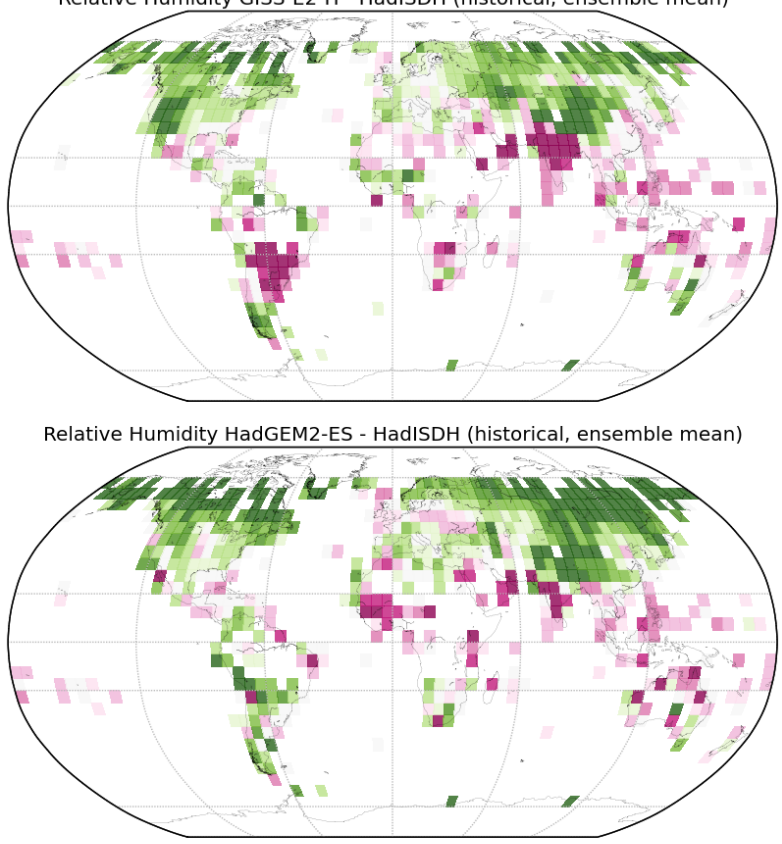

Relative Humidity NorESM1-M - HadISDH (historical, ensemble mean)
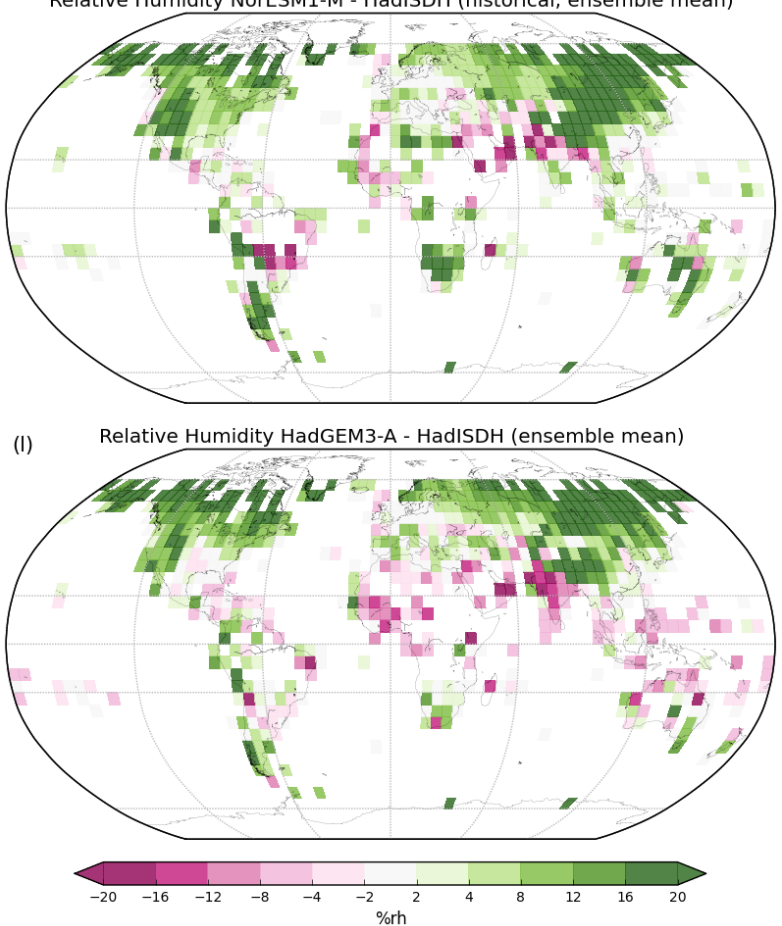

Figure S16. Climatological differences between models and observations at the gridbox scale for relative humidity. All climatologies have been calculated over the 1981 to 2010 period using identical spatio-temporal coverage to HadISDH. 

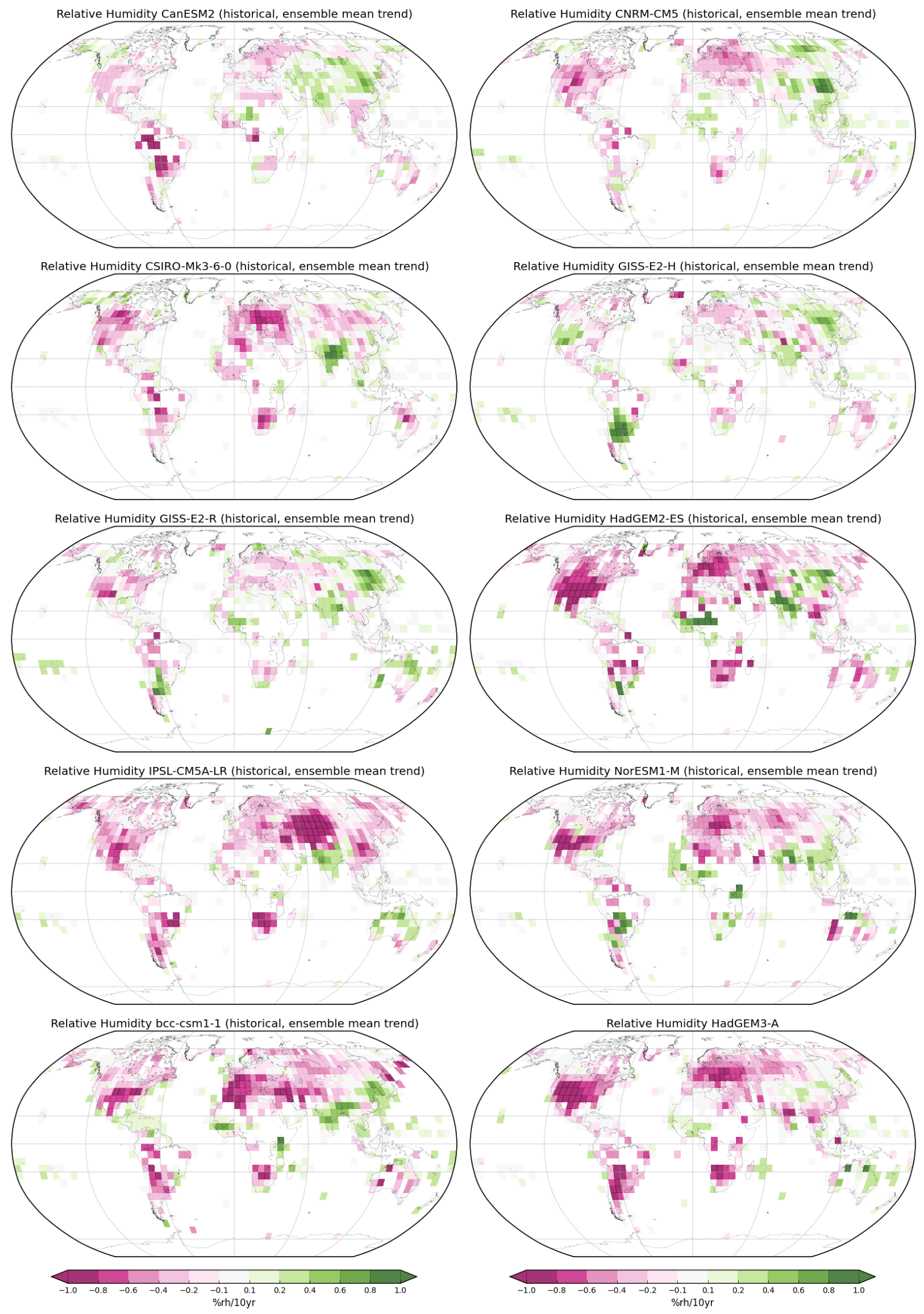

Figure S17. Linear trends of the CMIP5 models at the gridbox scale for relative humidity. All trends have been calculated using the median of pairwise slopes method, with identical spatio-temporal coverage to HadISDH. 


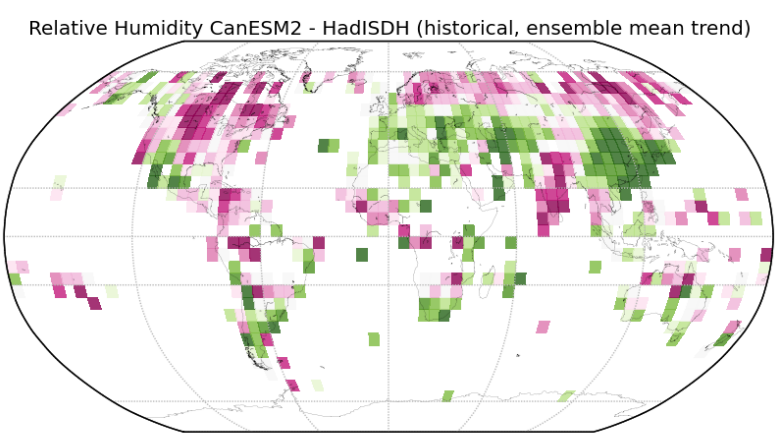

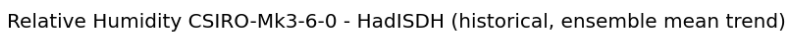

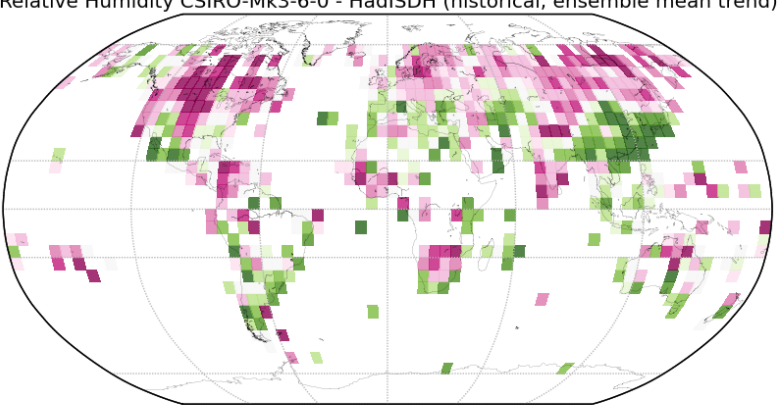

Relative Humidity GISS-E2-R - HadISDH (historical, ensemble mean trend)

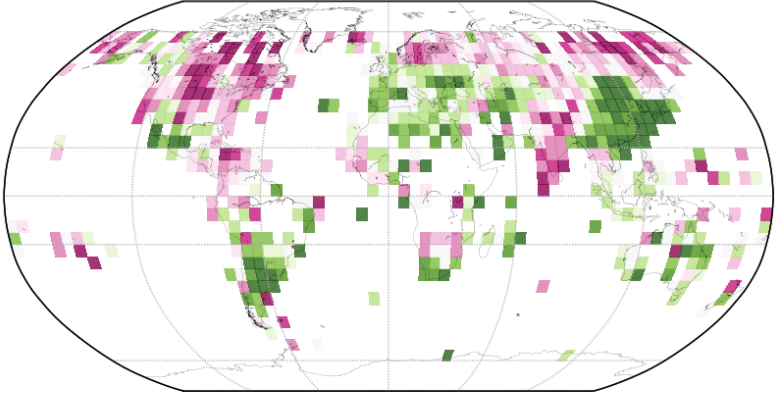

Relative Humidity IPSL-CM5A-LR - HadISDH (historical, ensemble mean trend)

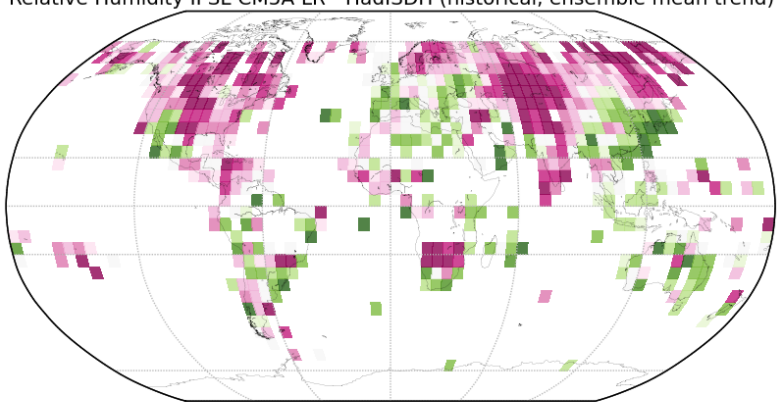

Relative Humidity bcc-csm1-1 - HadISDH (historical, ensemble mean trend)

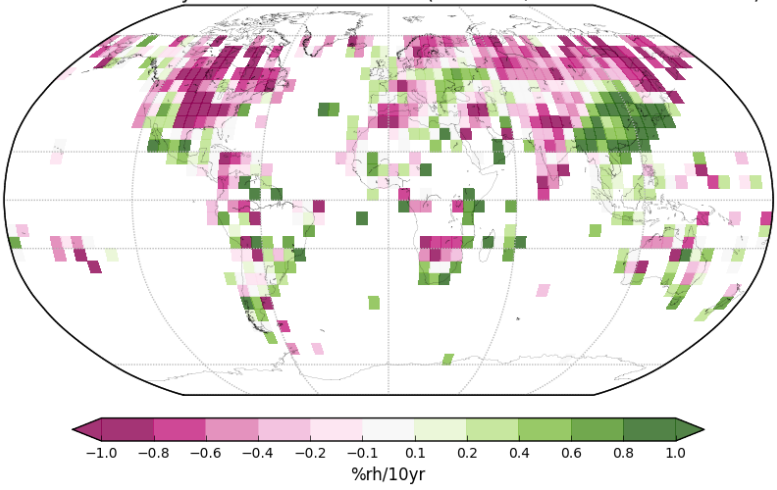

Relative Humidity CNRM-CM5 - HadISDH (historical, ensemble mean trend)

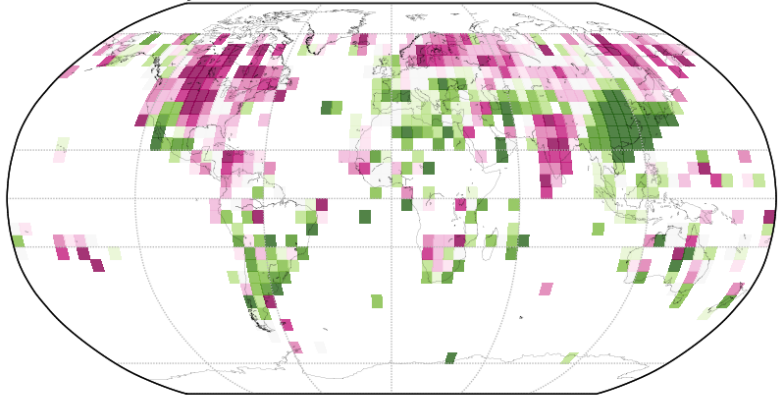

Relative Humidity GISS-E2-H - HadISDH (historical, ensemble mean trend)

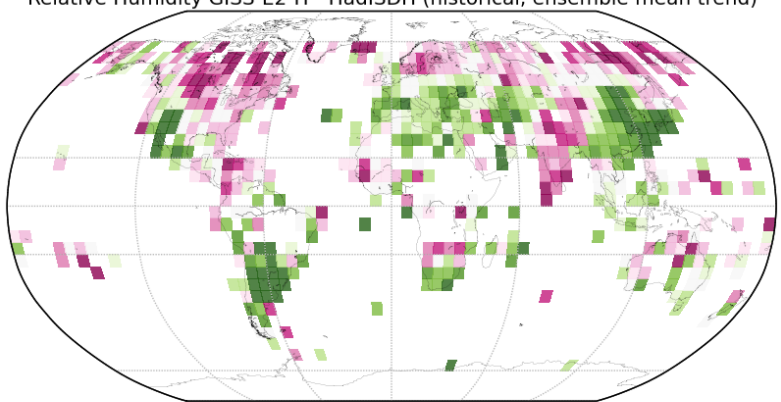

Relative Humidity HadGEM2-ES - HadISDH (historical, ensemble mean trend)

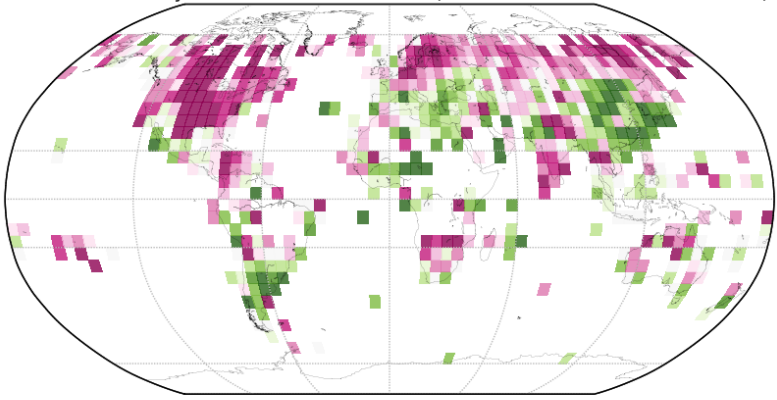

Relative Humidity NorESM1-M - HadISDH (historical, ensemble mean trend)
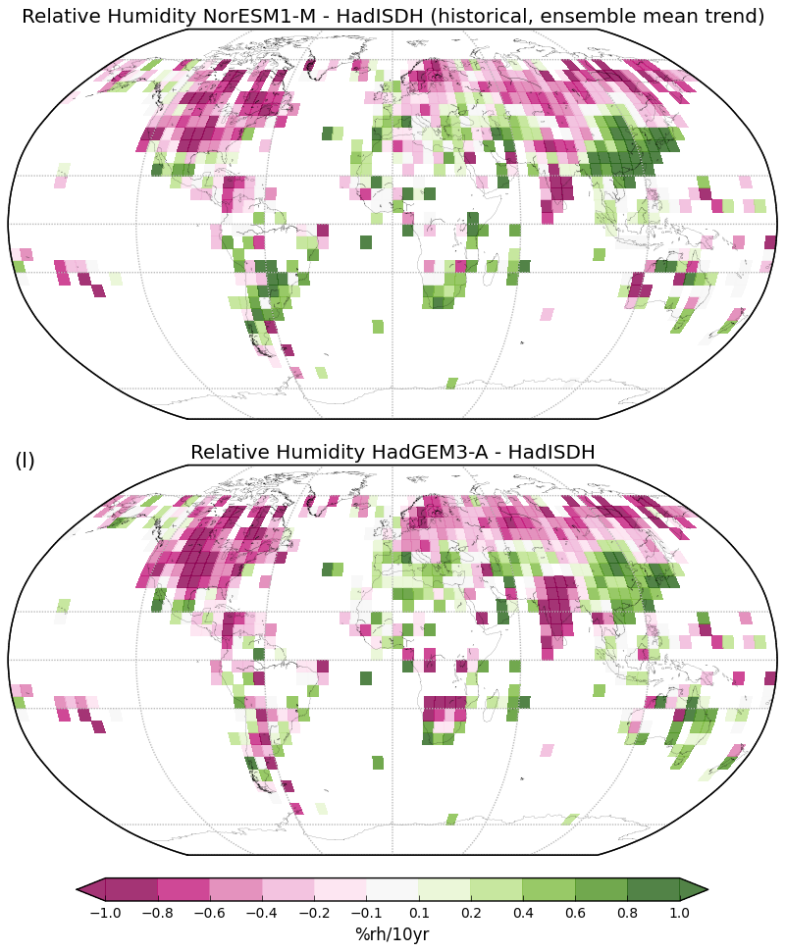

Figure S18. Linear trend differences of the CMIP5 models from the observations at the gridbox scale for relative humidity. All trends have been calculated using the median of pairwise slopes method, with identical spatio-temporal coverage to HadISDH. 


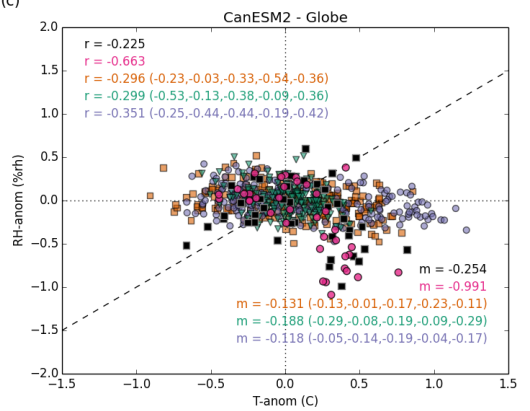

(e)
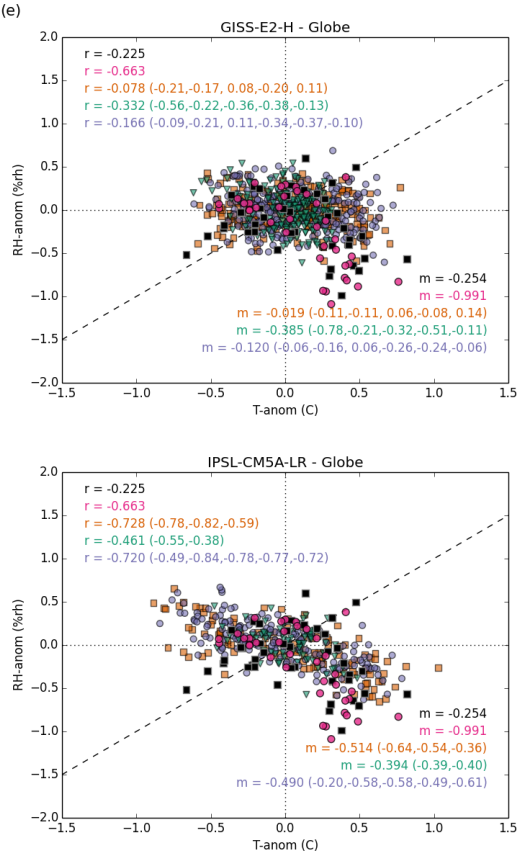

(a)
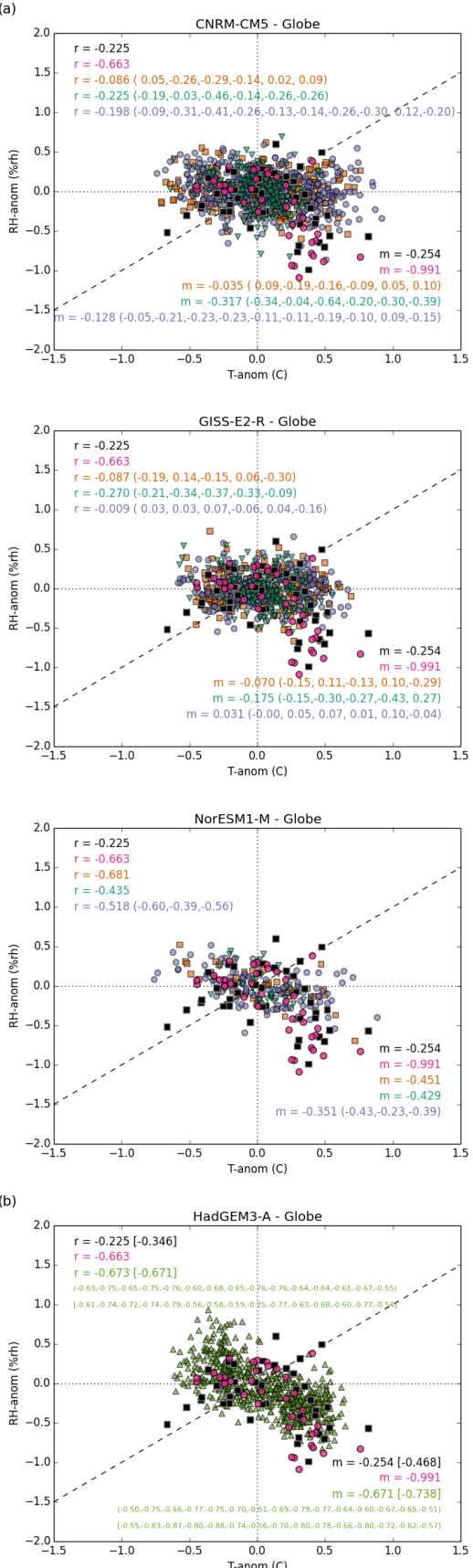

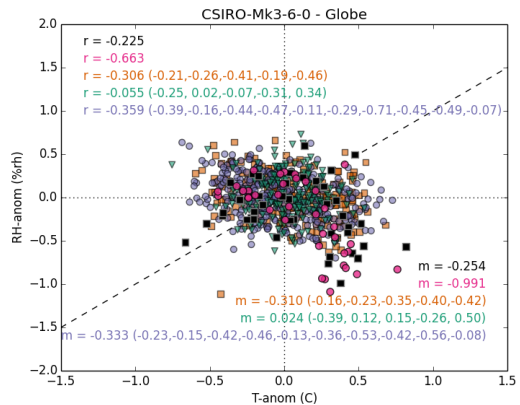

(g)
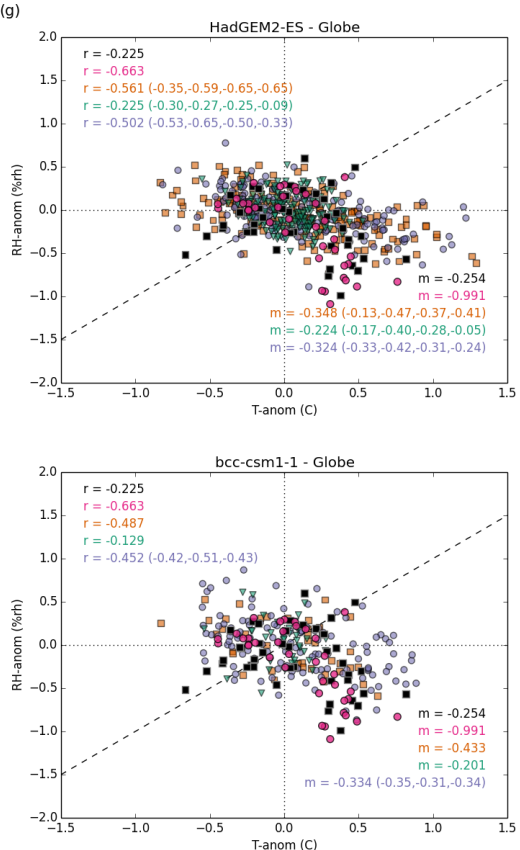

Figure S19. Relationships in global average temperature and relative humidity for CMIP5 models compared to HadISDH and ERA-Interim. Correlations (r) are shown in the top left hand corner. The gradient of the line of best fit is given in the bottom right hand corner. HadISDH is shown in black. ERA-Interim is shown in magenta. Historical, historicalNAT and historicalGHG are shown in purple, green and orange respectively. The ensemble mean value is given for each model with individual member values in parentheses. Values in square brackets are calculated using data matched to the coverage of ERA-Interim, for both HadISDH and HadGEM3-A. 

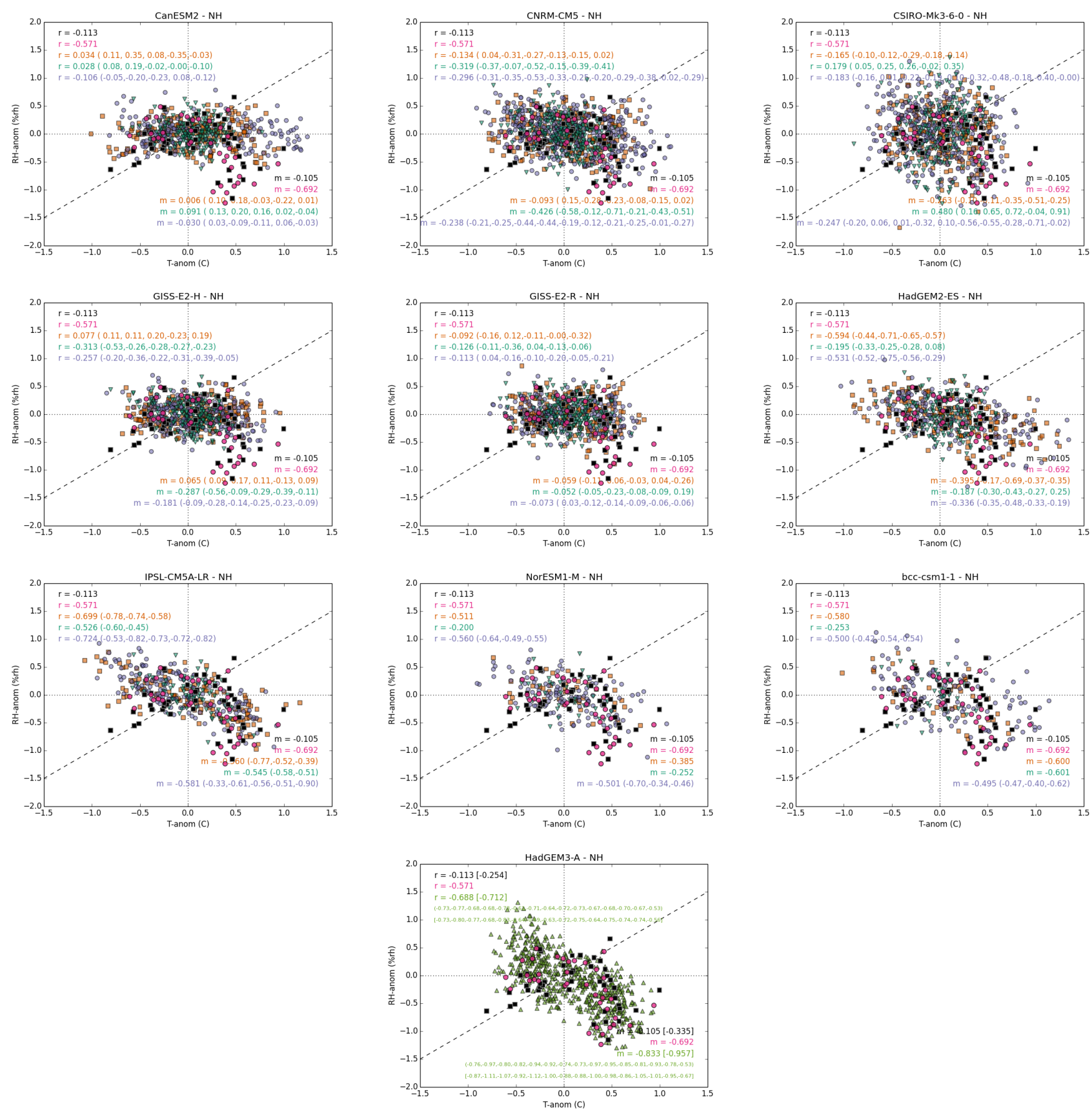

Figure S20. Relationships in Northern Hemisphere average temperature and relative humidity for CMIP5 models compared to HadISDH and ERA-Interim. Correlations (r) are shown in the top left hand corner. The gradient of the line of best fit is given in the bottom right hand corner. HadISDH is shown in black. ERA-Interim is shown in magenta. Historical, historicalNAT and historicalGHG are shown in purple, green and orange respectively. The ensemble mean value is given for each model with individual member values in parentheses. Values in square brackets are calculated using data matched to the coverage of ERA-Interim, for both HadISDH and HadGEM3-A. 

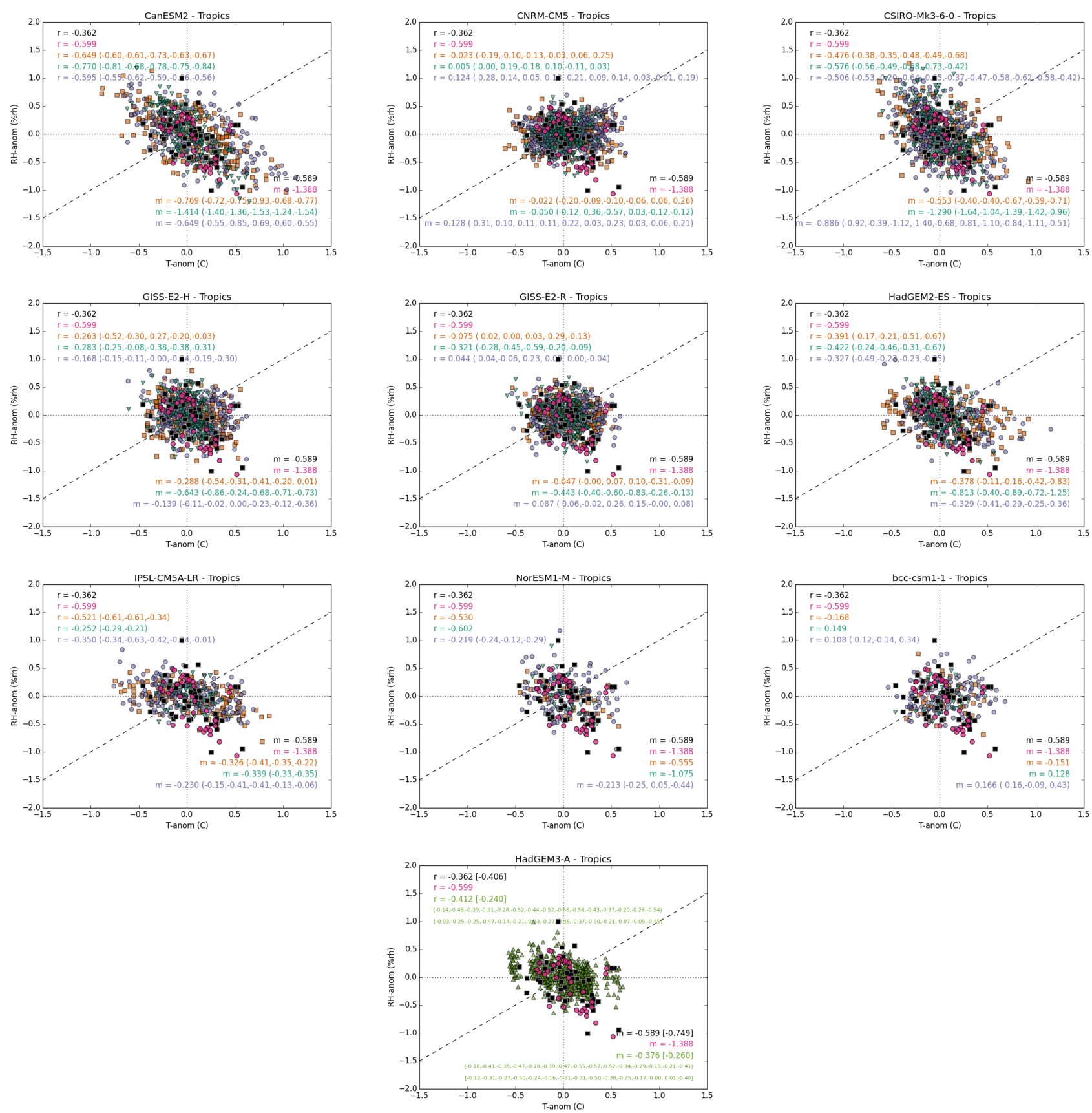

Figure S21. Relationships in tropical average temperature and relative humidity for CMIP5 models compared to HadISDH and ERAInterim. Correlations (r) are shown in the top left hand corner. The gradient of the line of best fit is given in the bottom right hand corner. HadISDH is shown in black. ERA-Interim is shown in magenta. Historical, historicalNAT and historicalGHG are shown in purple, green and orange respectively. The ensemble mean value is given for each model with individual member values in parentheses. Values in square brackets are calculated using data matched to the coverage of ERA-Interim, for both HadISDH and HadGEM3-A. 


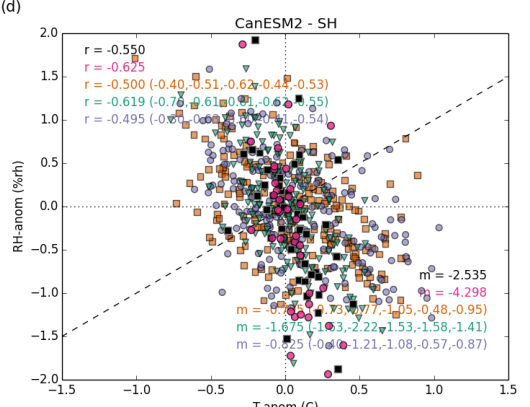

(f)
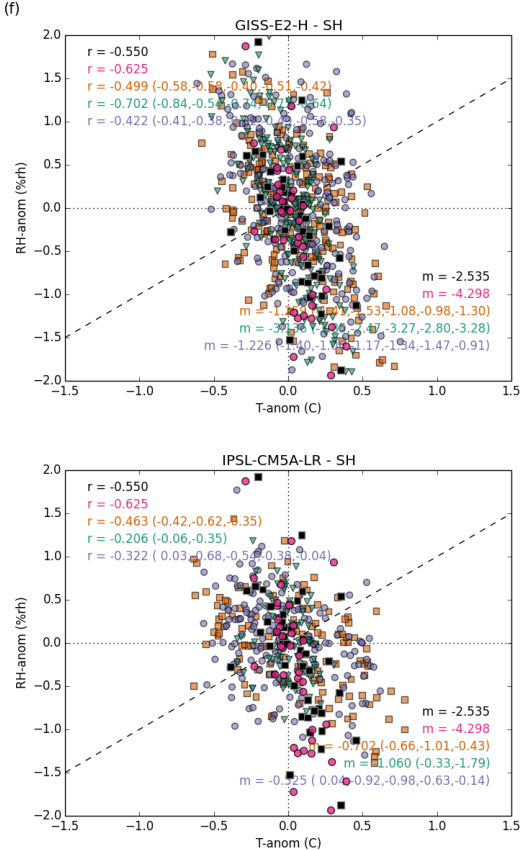

(b)
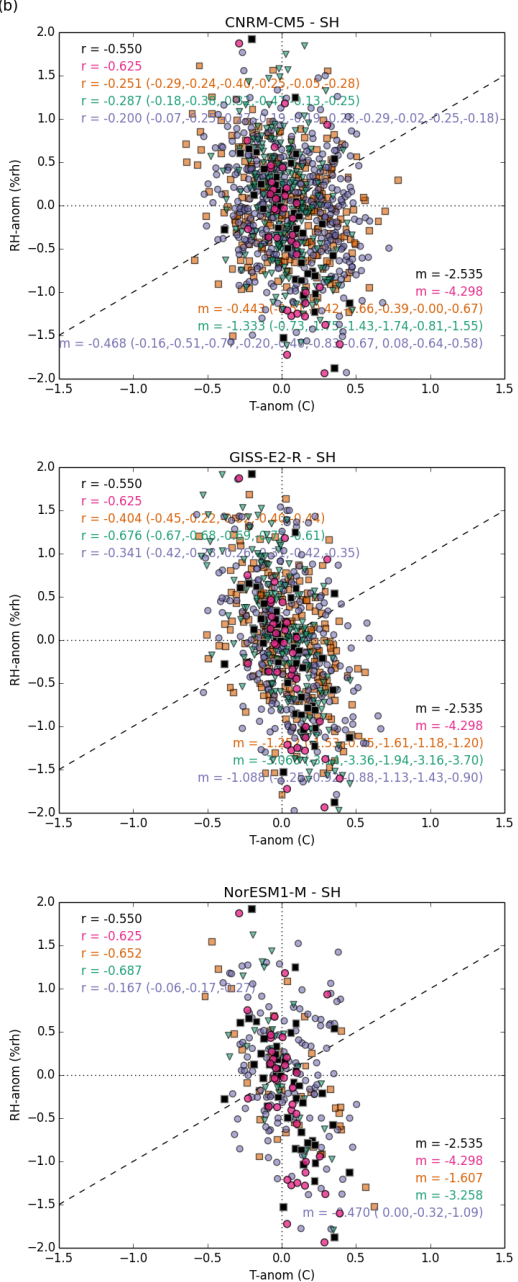

(d)

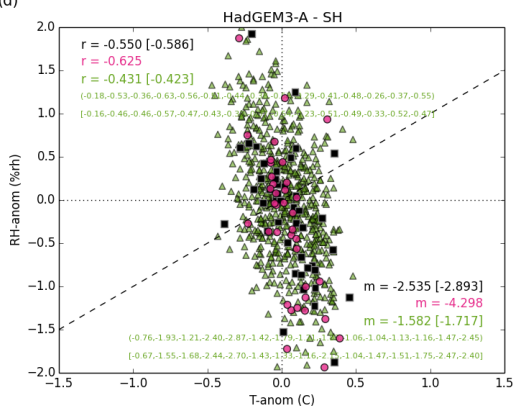

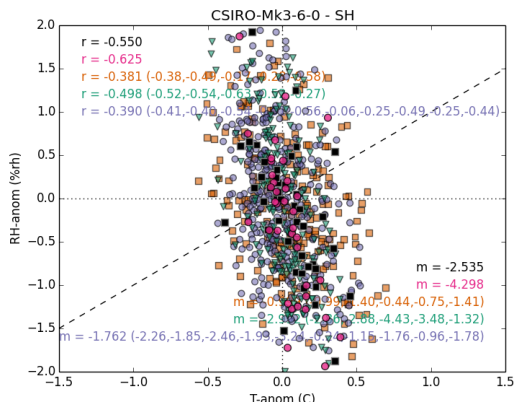

(h)
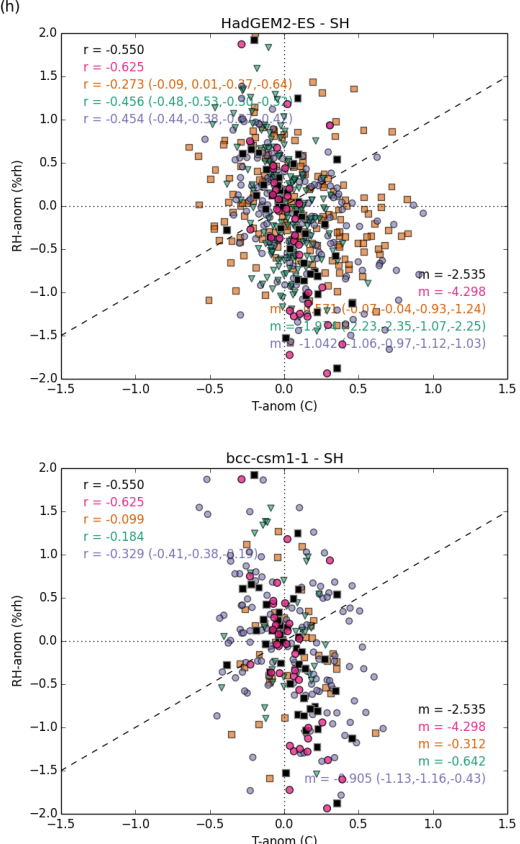

Figure S22. Relationships in Southern Hemisphere average temperature and relative humidity for CMIP5 models compared to HadISDH and ERA-Interim. Correlations (r) are shown in the top left hand corner. The gradient of the line of best fit is given in the bottom right hand corner. HadISDH is shown in black. ERA-Interim is shown in magenta. Historical, historicalNAT and historicalGHG are shown in purple, green and orange respectively. The ensemble mean value is given for each model with individual member values in parentheses. Values in square brackets are calculated using data matched to the coverage of ERA-Interim, for both HadISDH and HadGEM3-A. 


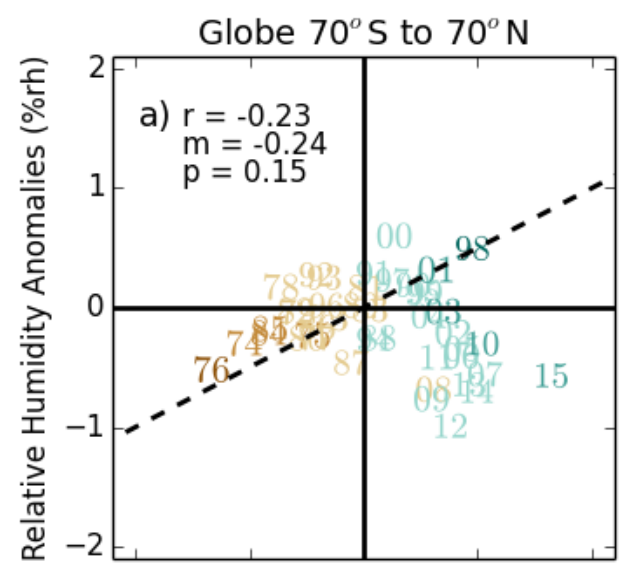

N. Hemisphere $20^{\circ} \mathrm{N}$ to $70^{\circ} \mathrm{N}$
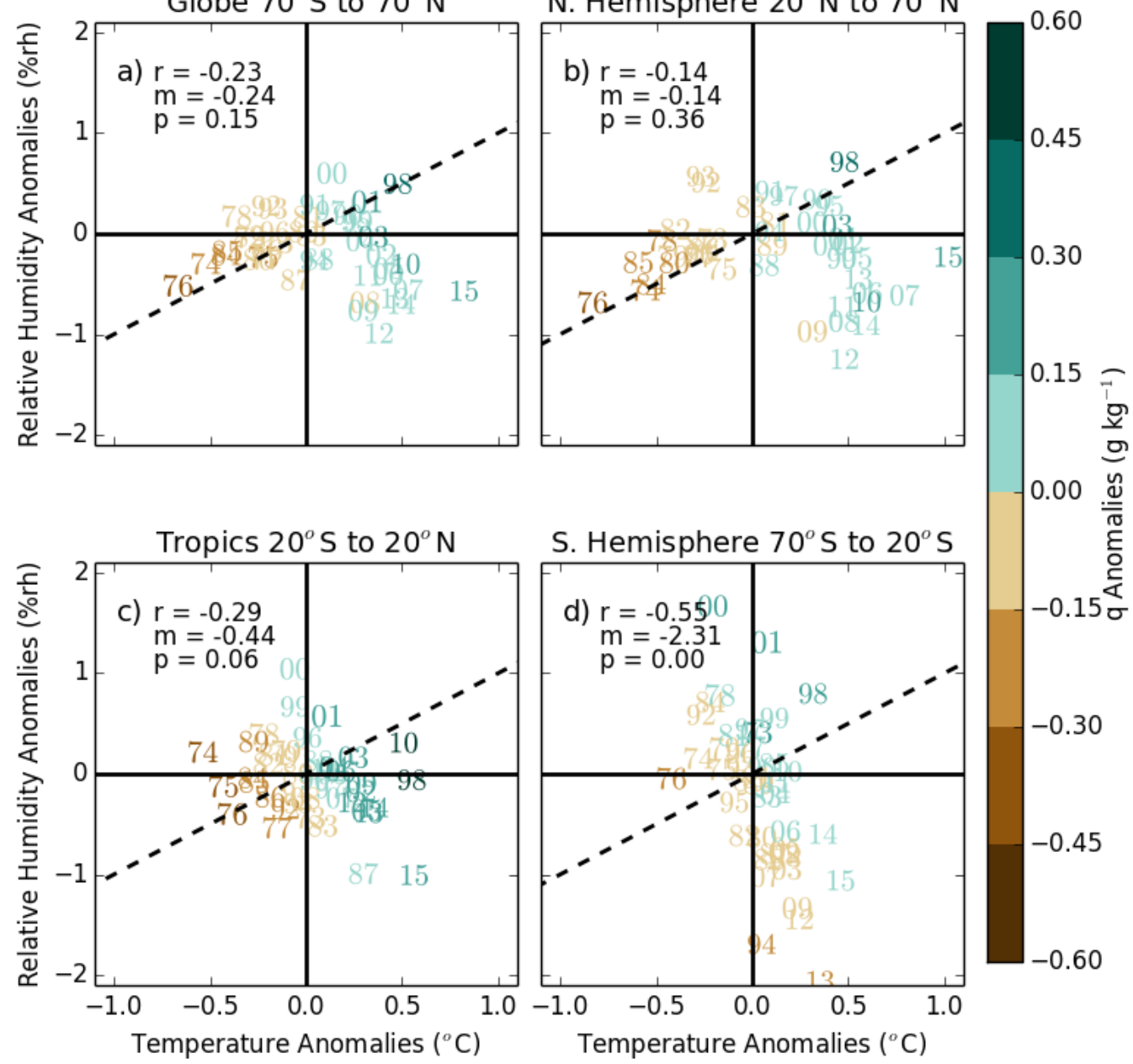

Figure S23. Temperature-relative humidity relationship, with the relative humidity anomaly as the colour scale, with the years as the points. The four zonal regions used in this analysis are shown in the four panels. 
Specific Humidity 

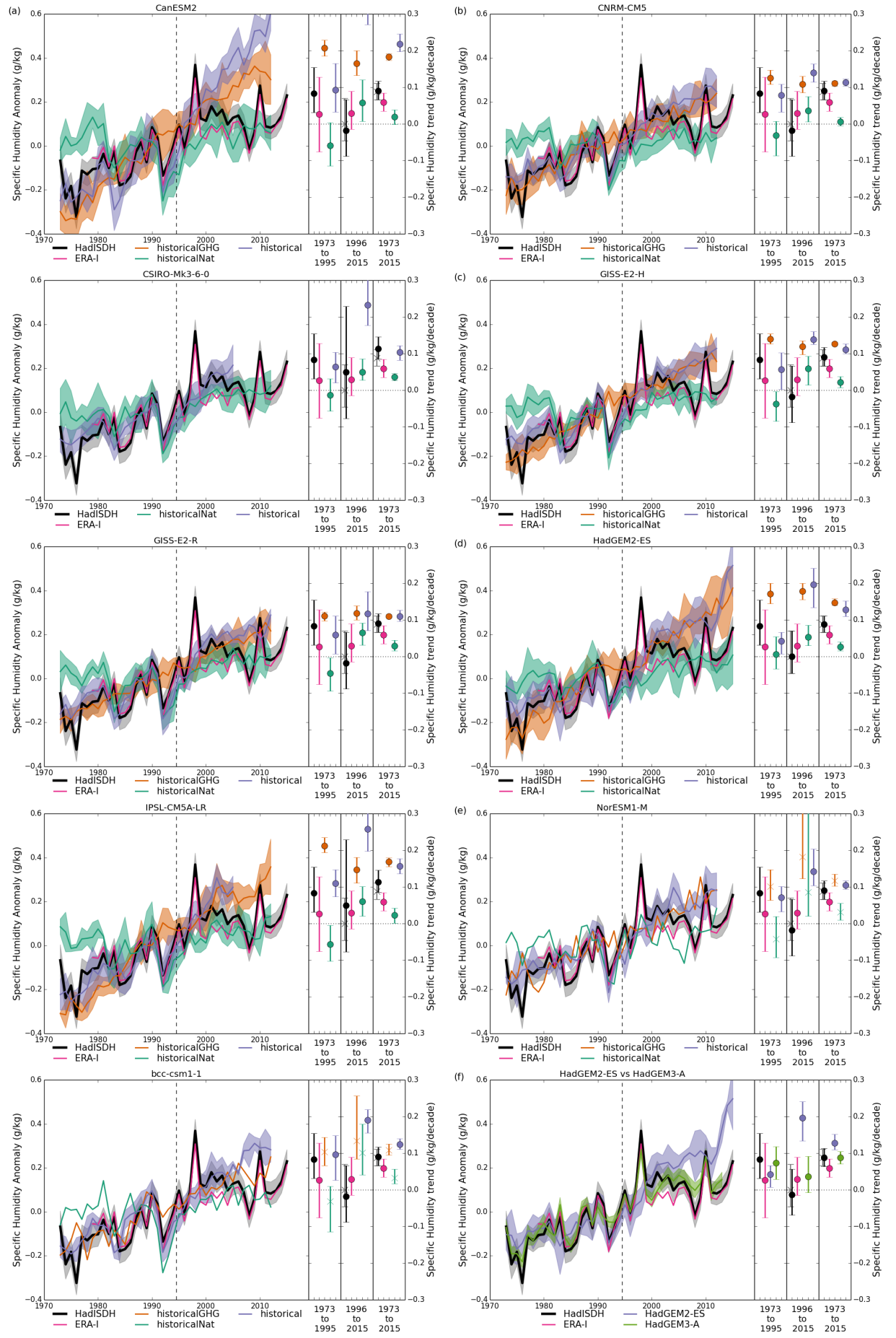

Figure S24. Global annual timeseries for specific humidity for the nine CMIP5 models and HadGEM3-A, using a climatology period of 1976-2005. HadISDH is shown by the thick black line, ERA-Interim by the magenta line and the historical, historicalNat and historicalGHG ensemble averages by the purple, green and orange lines respectively. The uncertainty ranges are shown using the coloured shading. The right-hand panels show the values of the linear trends for HadISDH, ERA-Interim and the ensemble averages of all three experiments for the early (1973-1994), late (1995-2015) and full periods. For the late and full period panels, the HadISDH trend is shown matching the temporal coverage of the historical model (circle) and its full coverage (cross). If there is only one ensemble member for the model, then the trend is marked with a cross rather than circle. 



Figure S25. Northern Hemisphere annual timeseries for specific humidity for the nine CMIP5 models and HadGEM3-A, using a climatology period of 1976-2005. HadISDH is shown by the thick black line, ERA-Interim by the magenta line and the historical, historicalNat and historicalGHG ensemble averages by the purple, green and orange lines respectively. The uncertainty ranges are shown using the coloured shading. The right-hand panels show the values of the linear trends for HadISDH, ERA-Interim and the ensemble averages of all three experiments for the early (1973-1994), late (1995-2015) and full periods. For the late and full period panels, the HadISDH trend is shown matching the temporal coverage of the historical model (circle) and its full coverage (cross). If there is only one ensemble member for the model, then the trend is marked with a cross rather than circle. 

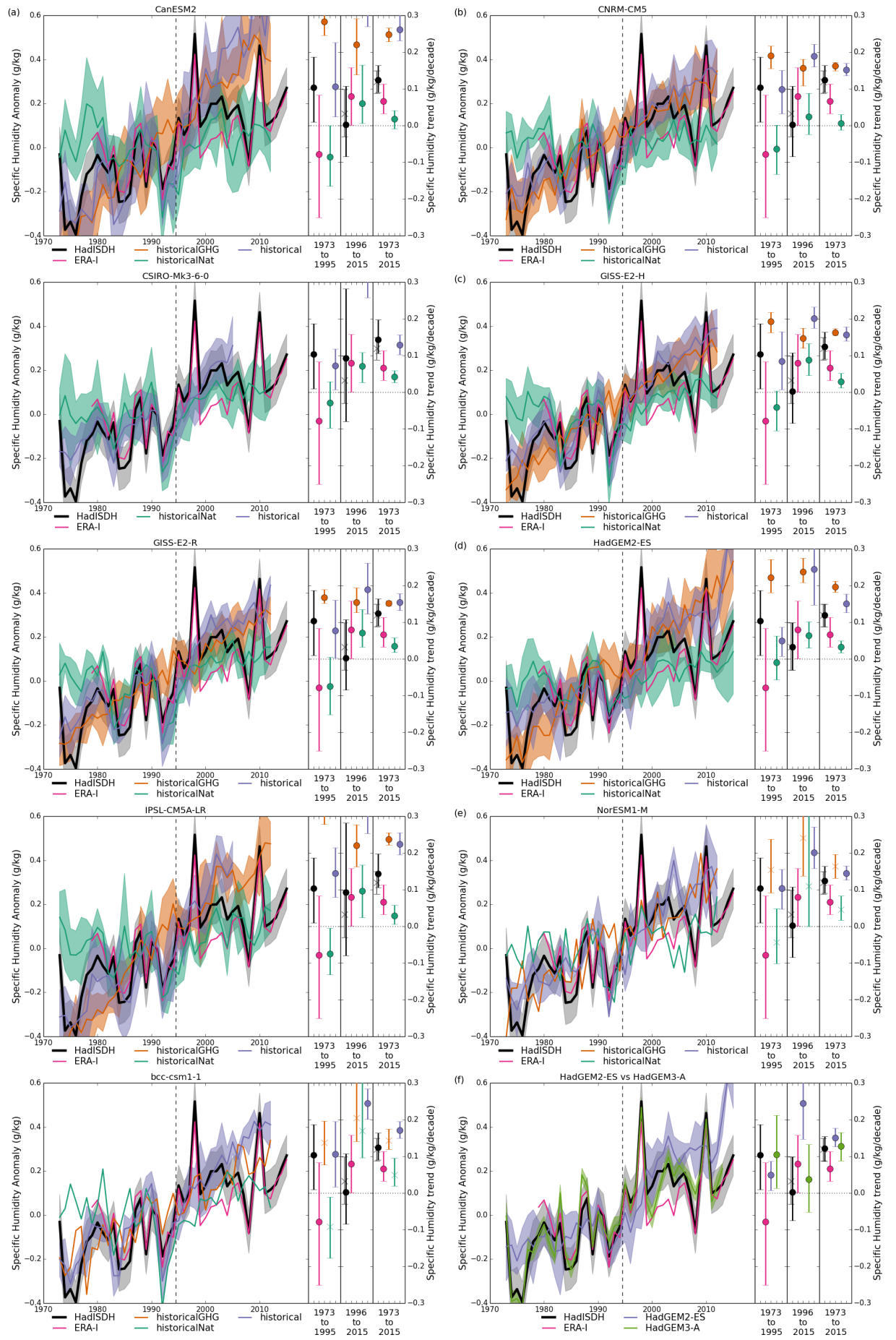

Figure S26. Tropical annual timeseries for specific humidity for the nine CMIP5 models and HadGEM3-A, using a climatology period of 1976-2005. HadISDH is shown by the thick black line, ERA-Interim by the magenta line and the historical, historicalNat and historicalGHG ensemble averages by the purple, green and orange lines respectively. The uncertainty ranges are shown using the coloured shading. The right-hand panels show the values of the linear trends for HadISDH, ERA-Interim and the ensemble averages of all three experiments for the early (1973-1994), late (1995-2015) and full periods. For the late and full period panels, the HadISDH trend is shown matching the temporal coverage of the historical model (circle) and its full coverage (cross). If there is only one ensemble member for the model, then the trend is marked with a cross rather than circle. 

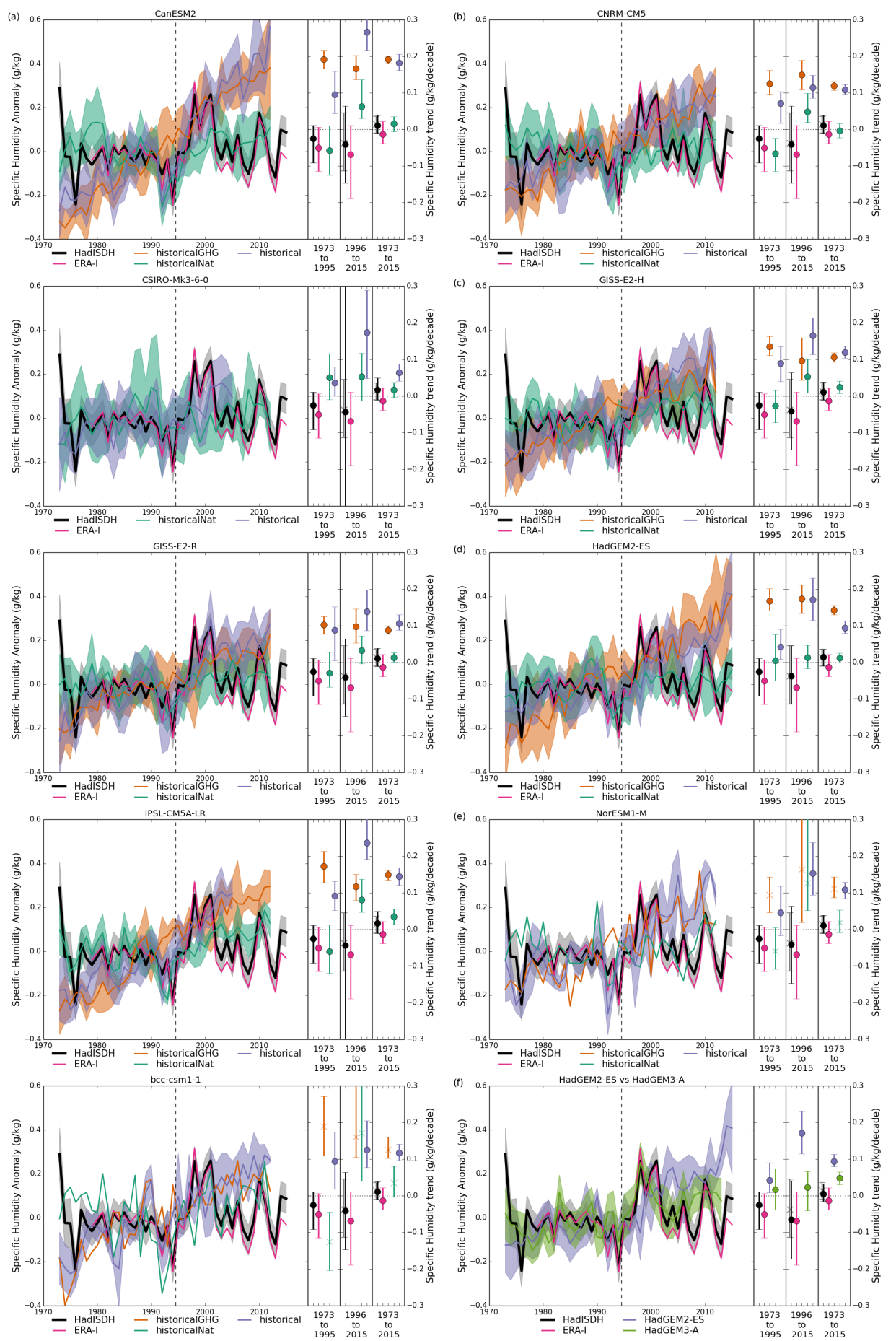

Figure S27. Southern Hemisphere annual timeseries for specific humidity for the nine CMIP5 models and HadGEM3-A, using a climatology period of 1976-2005. HadISDH is shown by the thick black line, ERA-Interim by the magenta line and the historical, historicalNat and historicalGHG ensemble averages by the purple, green and orange lines respectively. The uncertainty ranges are shown using the coloured shading. The right-hand panels show the values of the linear trends for HadISDH, ERA-Interim and the ensemble averages of all three experiments for the early (1973-1994), late (1995-2015) and full periods. For the late and full period panels, the HadISDH trend is shown matching the temporal coverage of the historical model (circle) and its full coverage (cross). If there is only one ensemble member for the model, then the trend is marked with a cross rather than circle. 

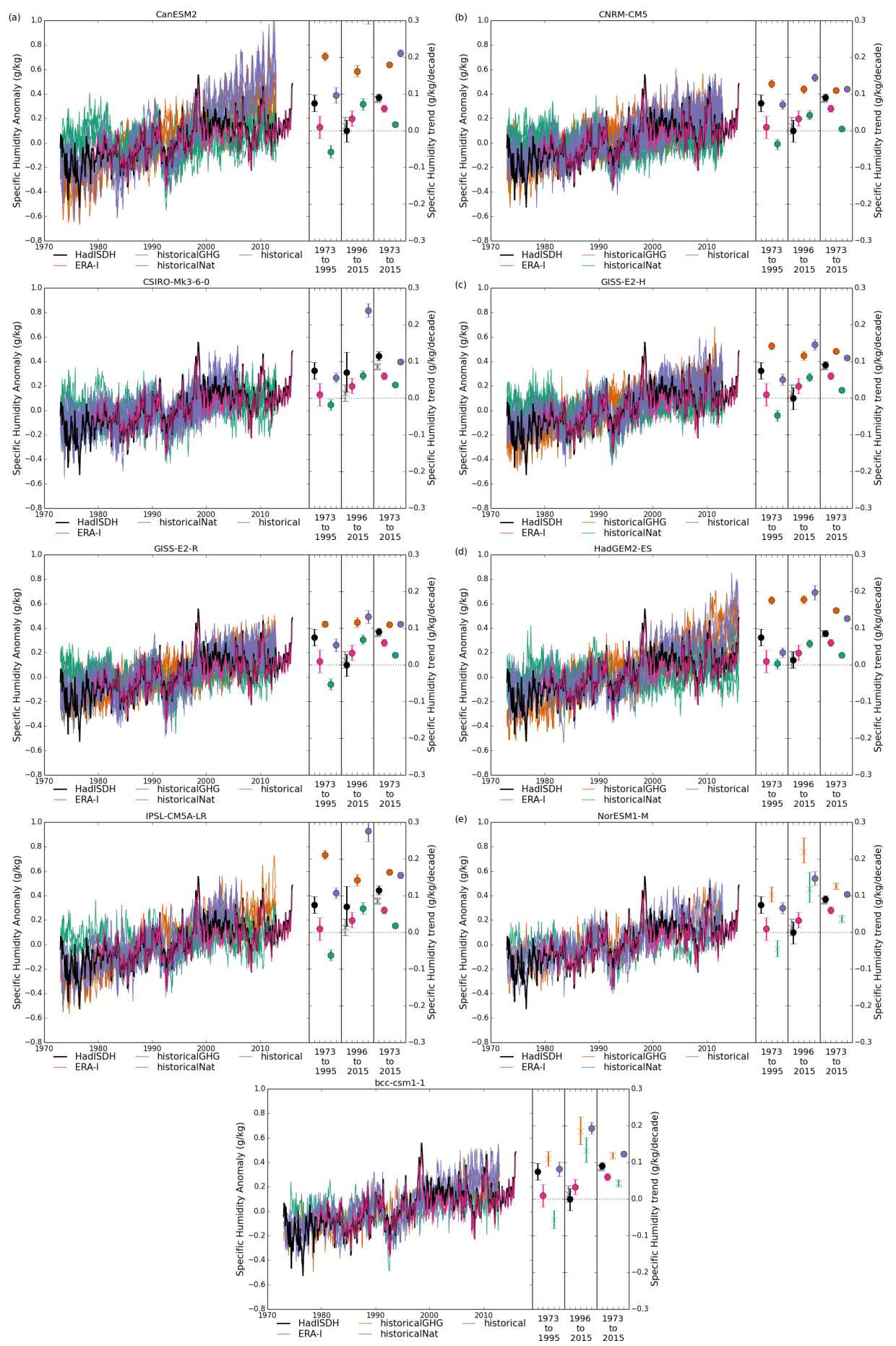

Figure S28. Global monthly timeseries for specific humidity for the nine CMIP5 models and HadGEM3-A, using a climatology period of 1976-2005. HadISDH is shown by the thick black line, ERA-Interim by the magenta line and the historical, historicalNat and historicalGHG ensemble members by the purple, green and orange lines respectively. he right-hand panels show the values of the linear trends for HadISDH, ERA-Interim and the ensemble averages of all three experiments for the early (1973-1994), late (1995-2015) and full periods. For the late and full period panels, the HadISDH trend is shown matching the temporal coverage of the historical model (circle) and its full coverage (cross). If there is only one ensemble member for the model, then the trend is marked with a cross rather than circle. 


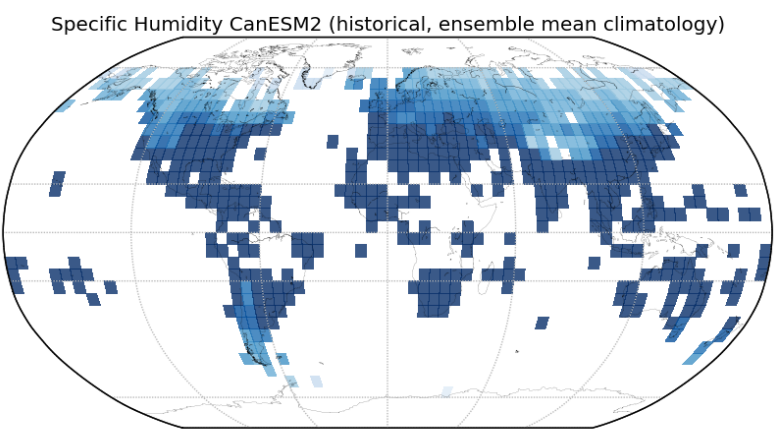

Specific Humidity CSIRO-Mk3-6-0 (historical, ensemble mean climatology)
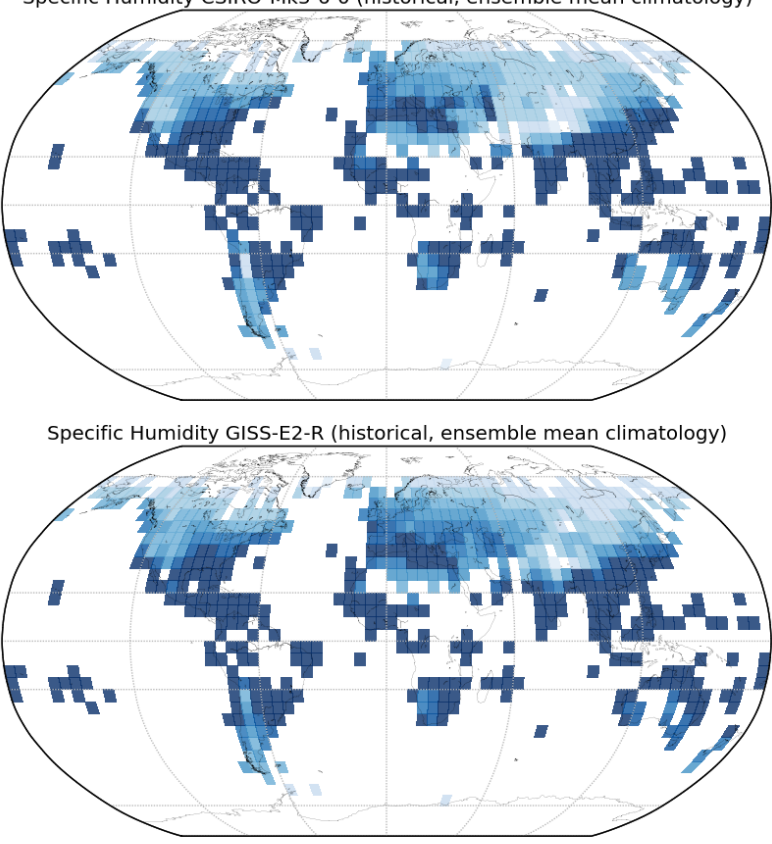

Specific Humidity IPSL-CM5A-LR (historical, ensemble mean climatology)

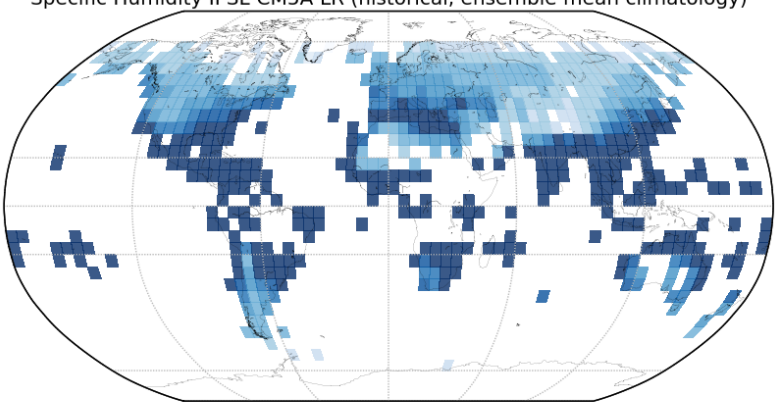

Specific Humidity bcc-csm1-1 (historical, ensemble mean climatology)

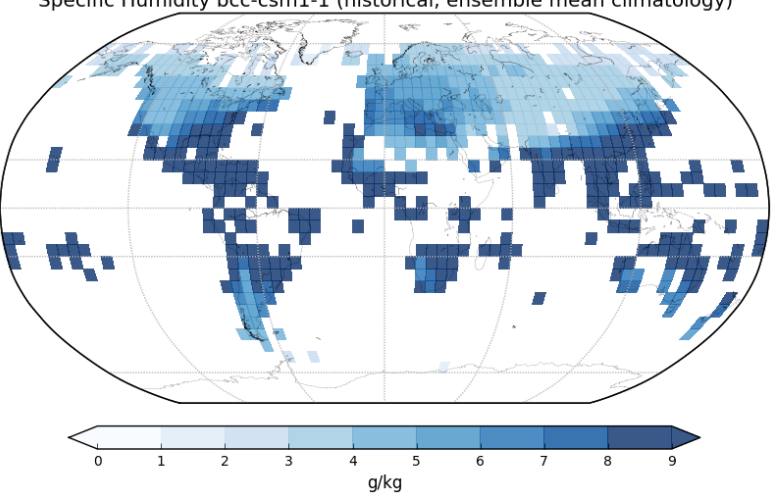

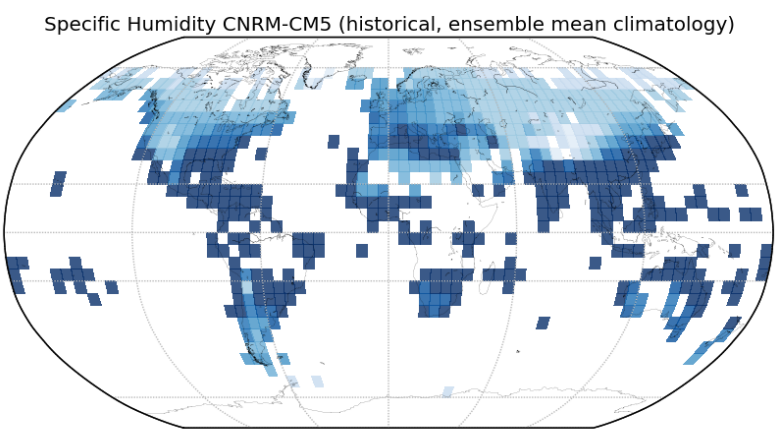

Specific Humidity GISS-E2-H (historical, ensemble mean climatology)

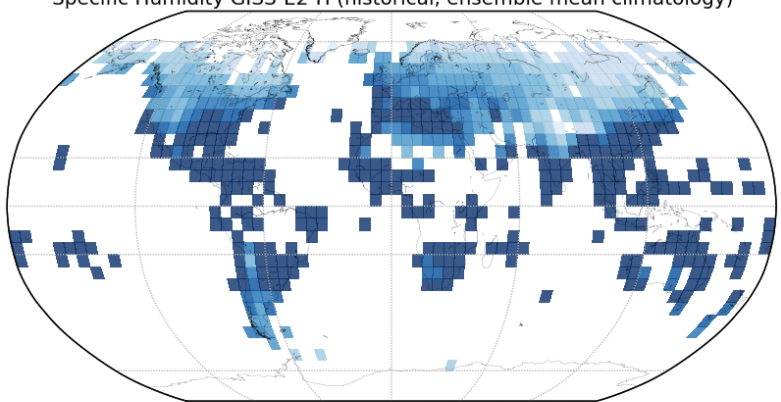

Specific Humidity HadGEM2-ES (historical, ensemble mean climatology)

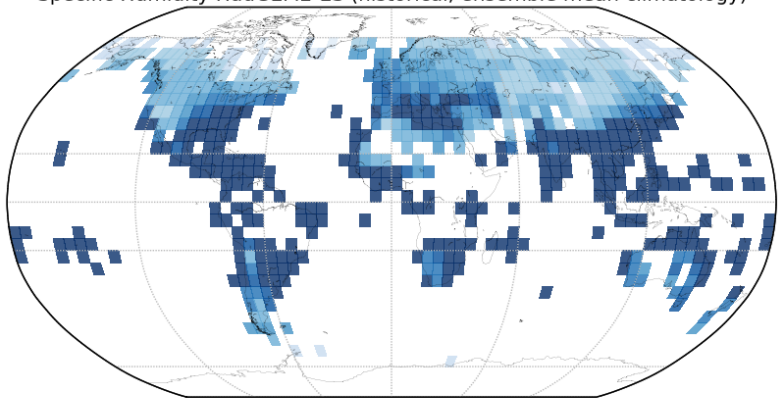

Specific Humidity NorESM1-M (historical, ensemble mean climatology)
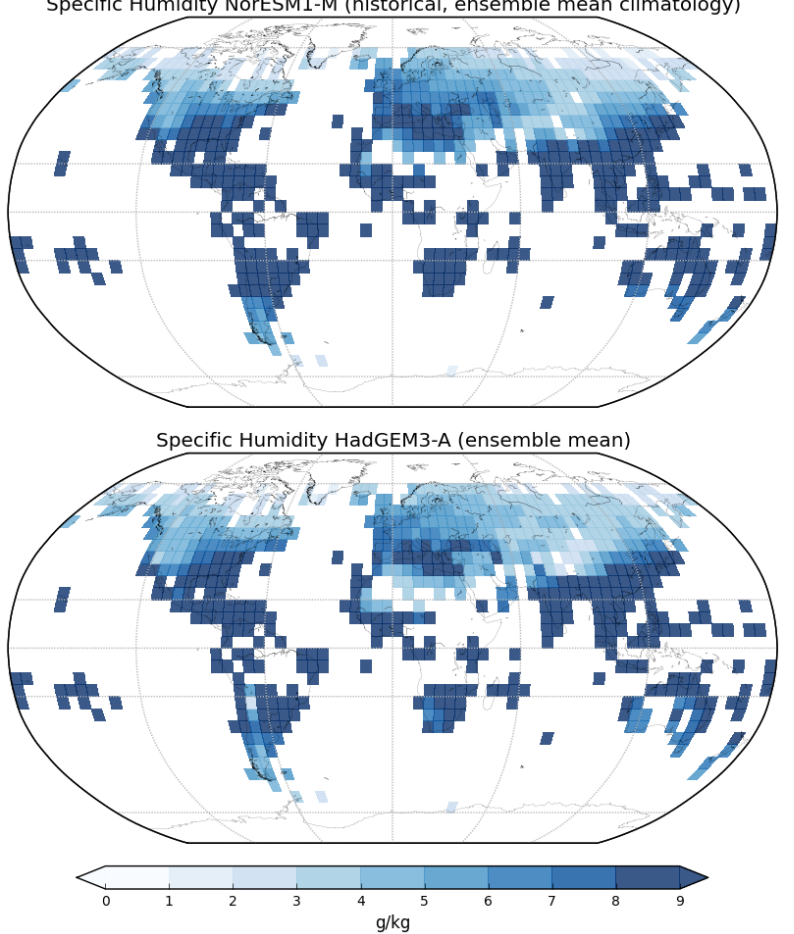

Figure S29. Specific humidity climatologies of the CMIP5 models at the gridbox scale. All climatologies have been calculated over the 1981 to 2010 period using identical spatio-temporal coverage to HadISDH. 


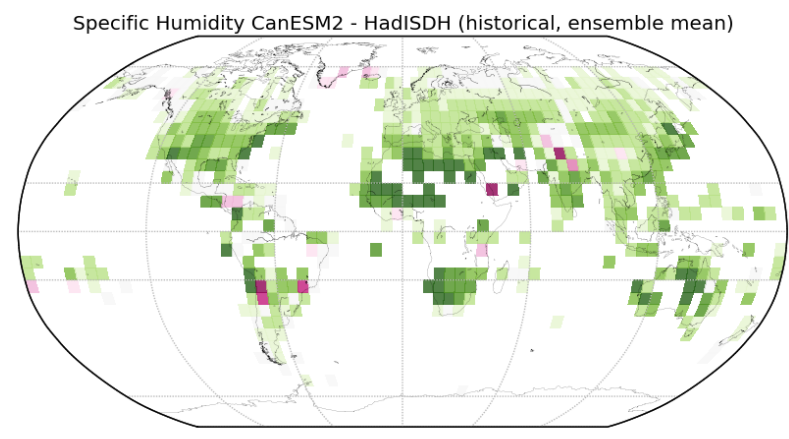

Specific Humidity CSIRO-Mk3-6-0 - HadISDH (historical, ensemble mean)
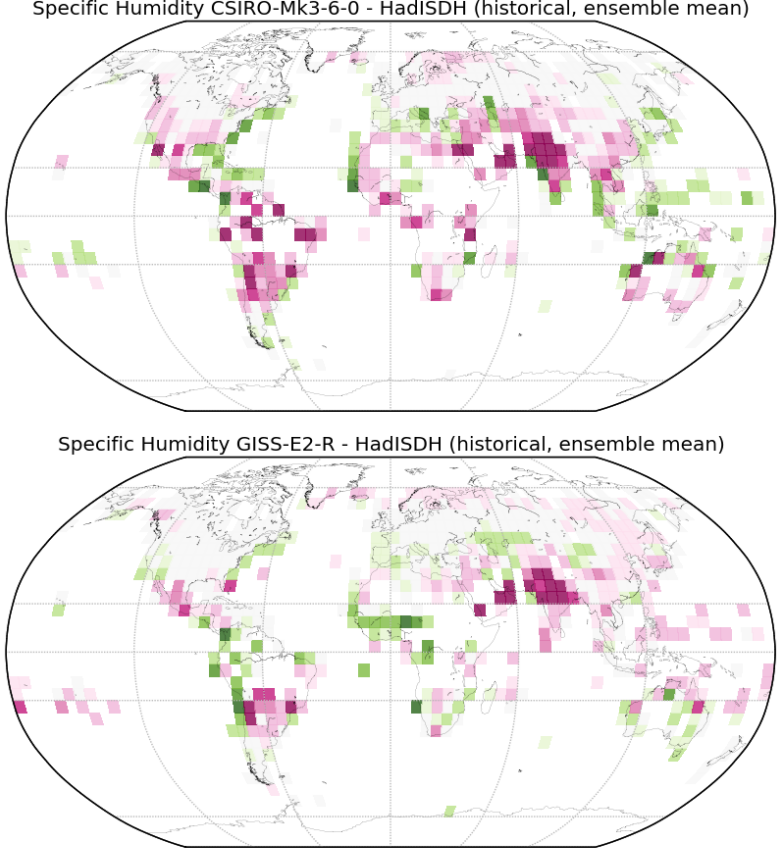

Specific Humidity IPSL-CM5A-LR - HadISDH (historical, ensemble mean)
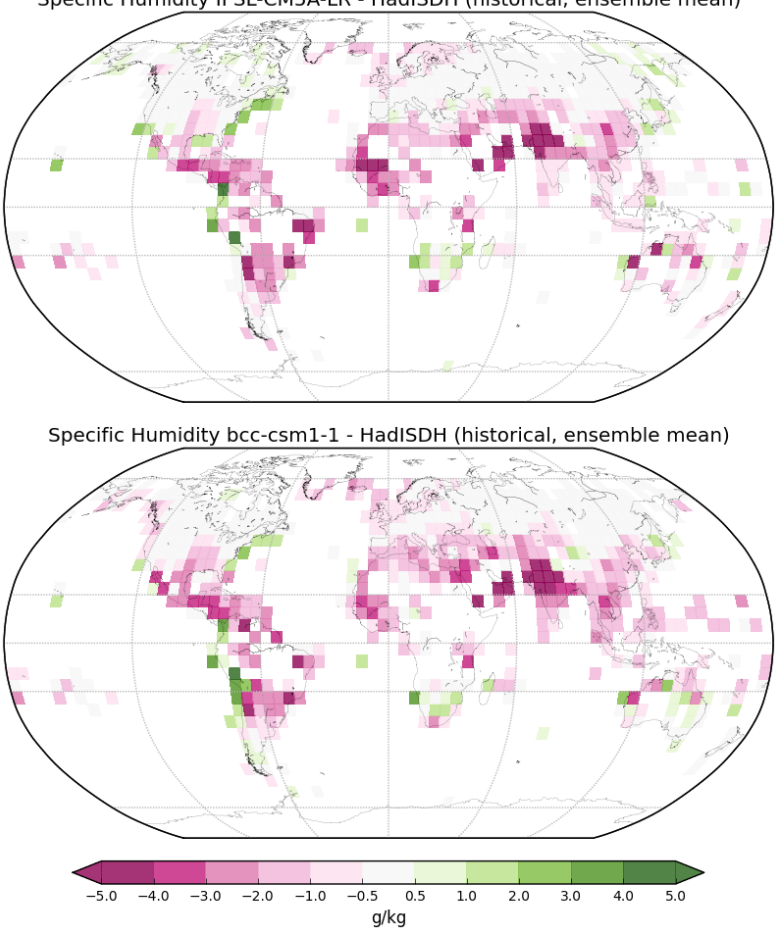

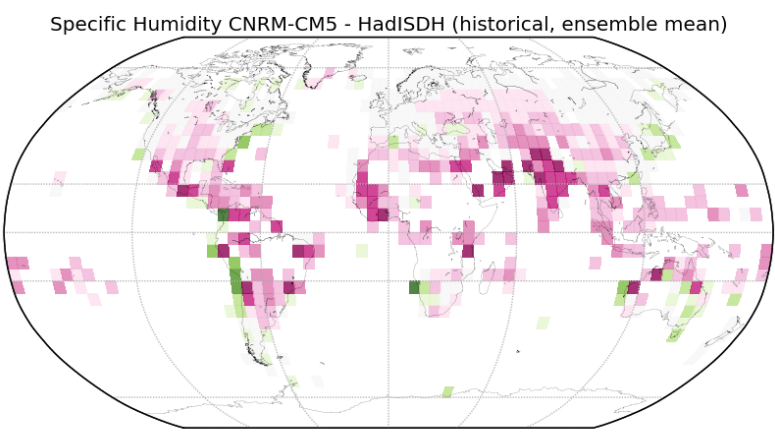

Specific Humidity GISS-E2-H - HadISDH (historical, ensemble mean)
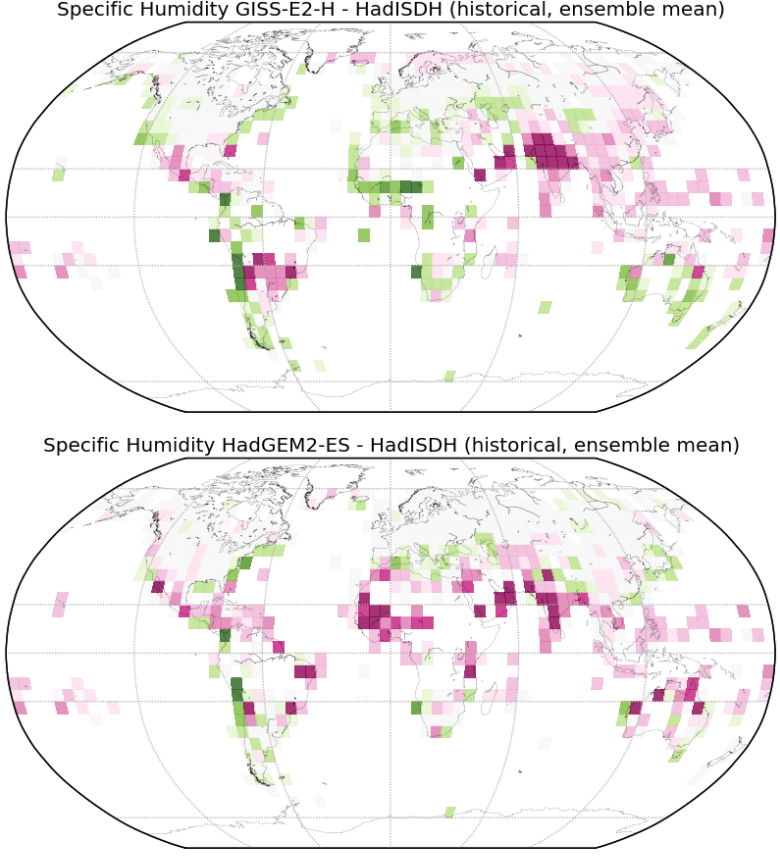

Specific Humidity NorESM1-M - HadISDH (historical, ensemble mean)

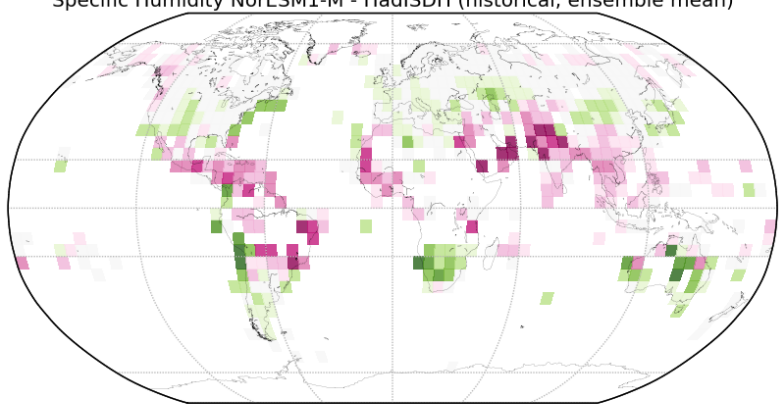

(k) Specific Humidity HadGEM3-A - HadISDH (ensemble mean)

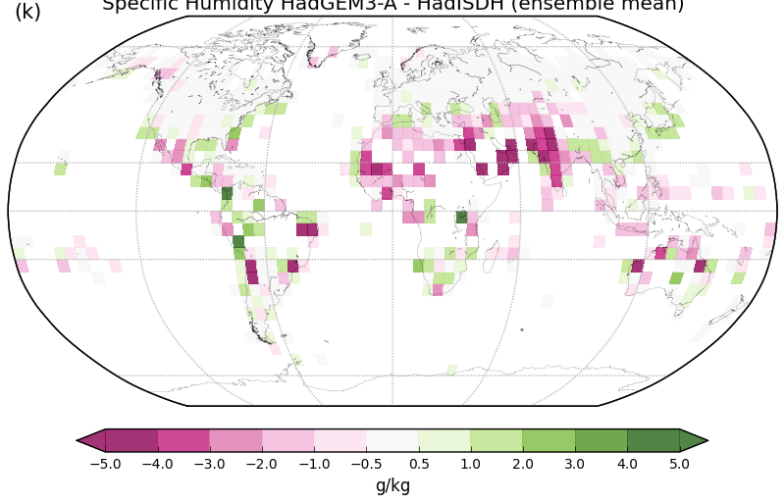

Figure S30. Climatological differences between models and observations at the gridbox scale for specific humidity. All climatologies have been calculated over the 1981 to 2010 period using identical spatio-temporal coverage to HadISDH. 

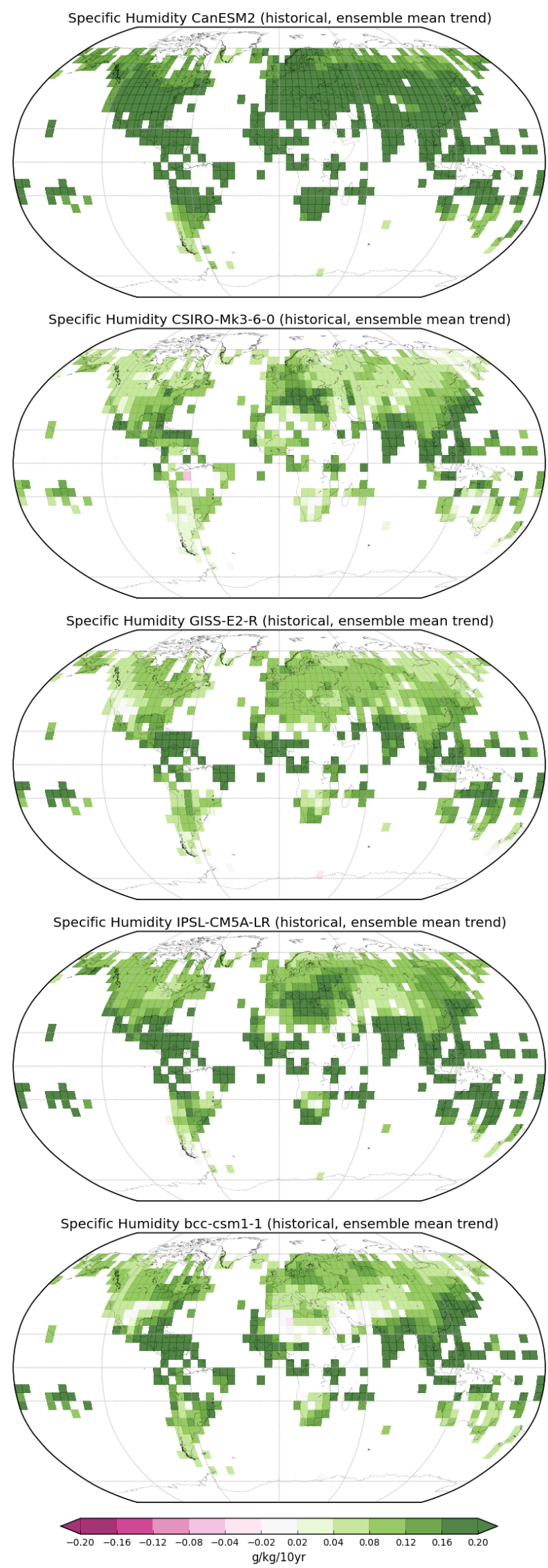
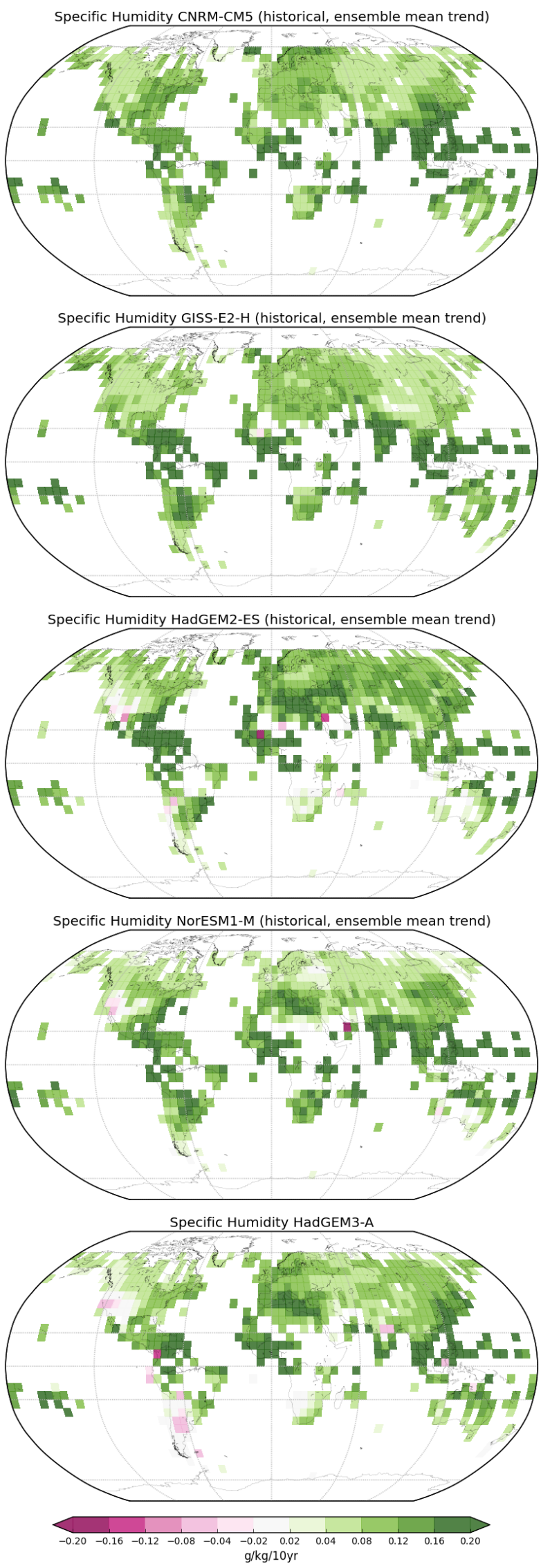

Figure S31. Linear trends of the CMIP5 models at the gridbox scale for specific humidity. All trends have been calculated using the median of pairwise slopes method, with identical spatio-temporal coverage to HadISDH. 


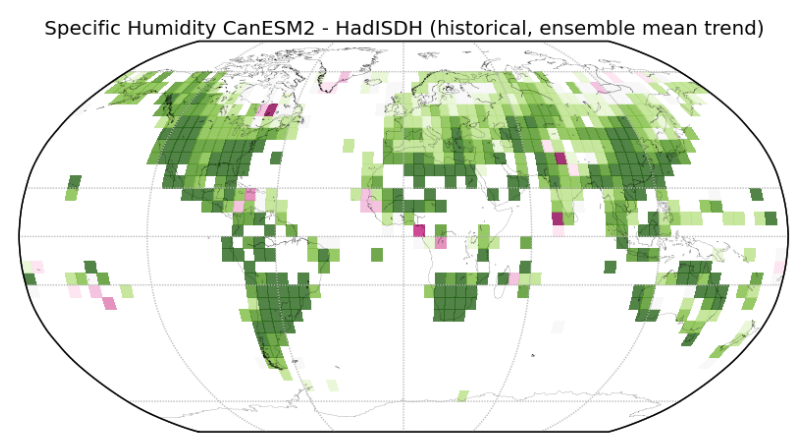

Specific Humidity CSIRO-Mk3-6-0 - HadISDH (historical, ensemble mean trend)

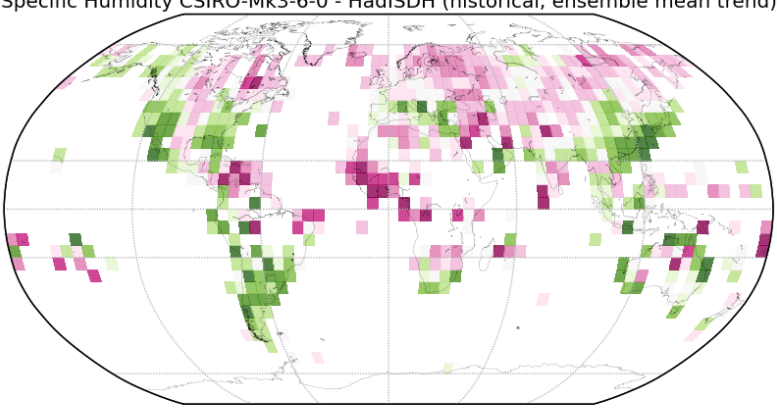

Specific Humidity GISS-E2-R - HadISDH (historical, ensemble mean trend)

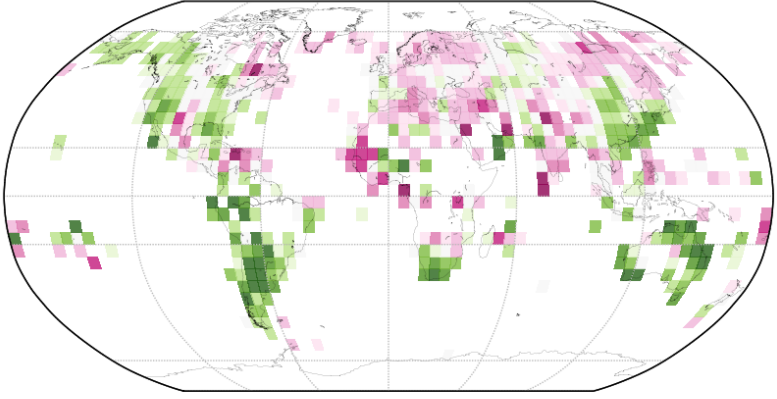

Specific Humidity IPSL-CM5A-LR - HadISDH (historical, ensemble mean trend)

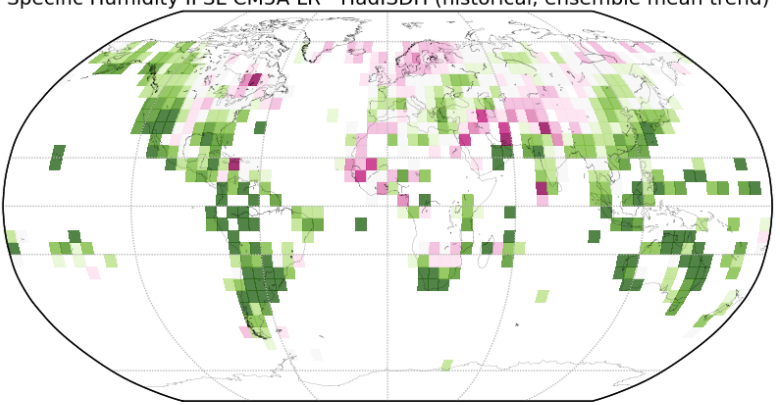

Specific Humidity bcc-csm1-1 - HadISDH (historical, ensemble mean trend)

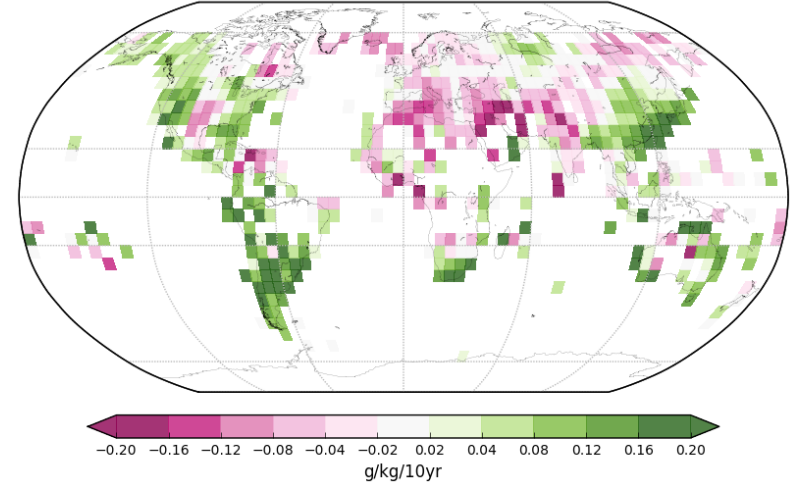

Specific Humidity CNRM-CM5 - HadISDH (historical, ensemble mean trend)

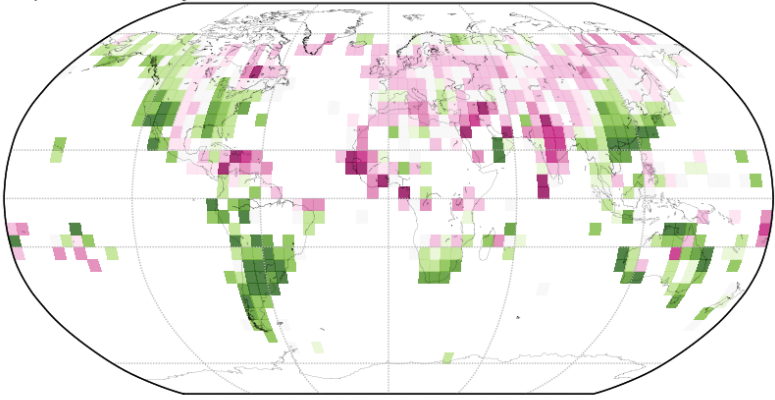

Specific Humidity GISS-E2-H - HadISDH (historical, ensemble mean trend)

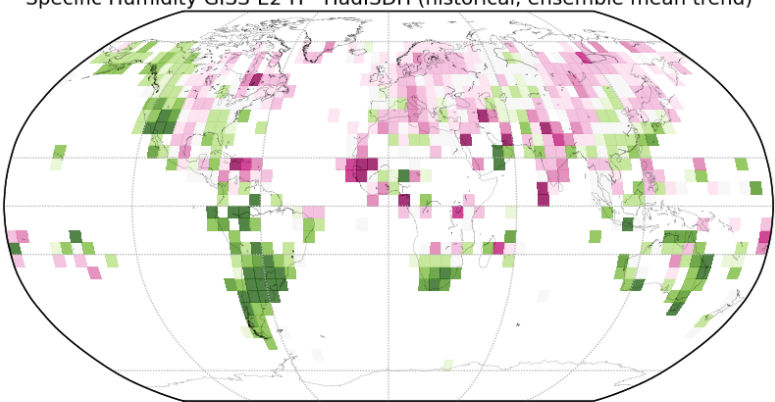

Specific Humidity HadGEM2-ES - HadISDH (historical, ensemble mean trend)

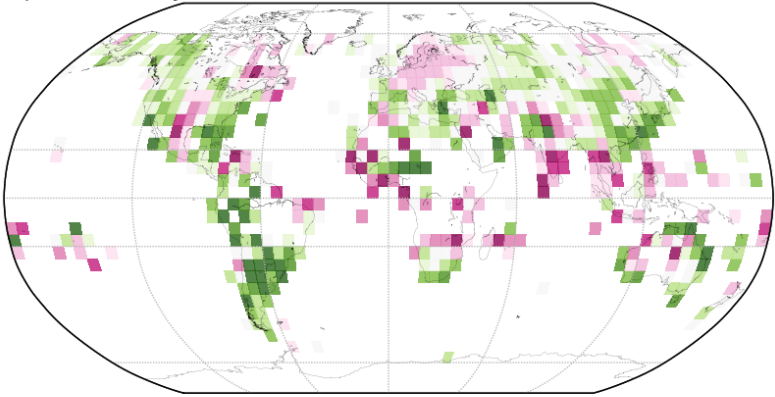

Specific Humidity NorESM1-M - HadISDH (historical, ensemble mean trend)
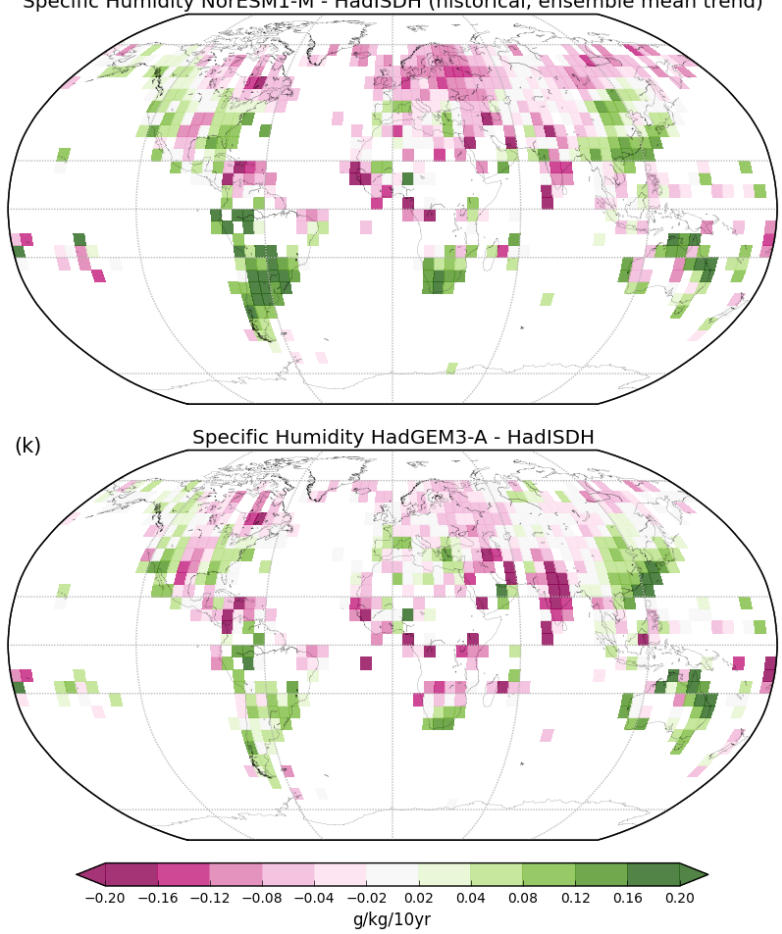

Figure S32. Linear trend differences of the CMIP5 models from the observations at the gridbox scale for specific humidity. All trends have been calculated using the median of pairwise slopes method, with identical spatio-temporal coverage to HadISDH. 


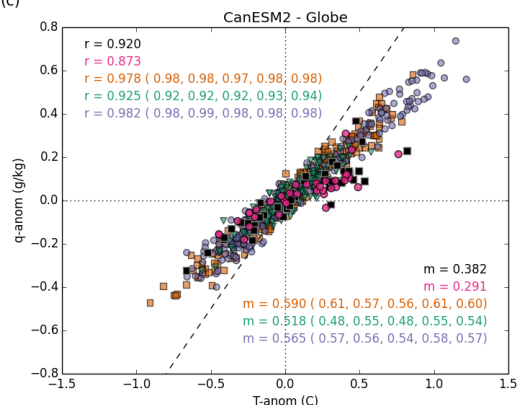

(e)
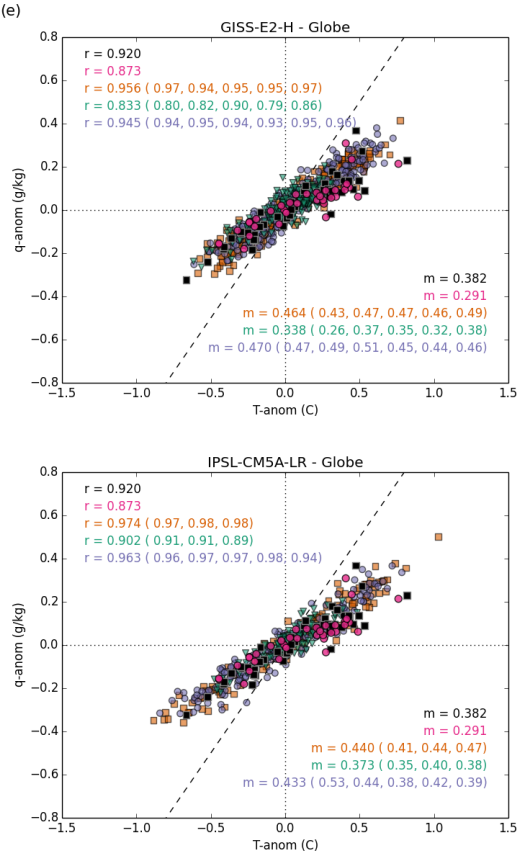

(a)
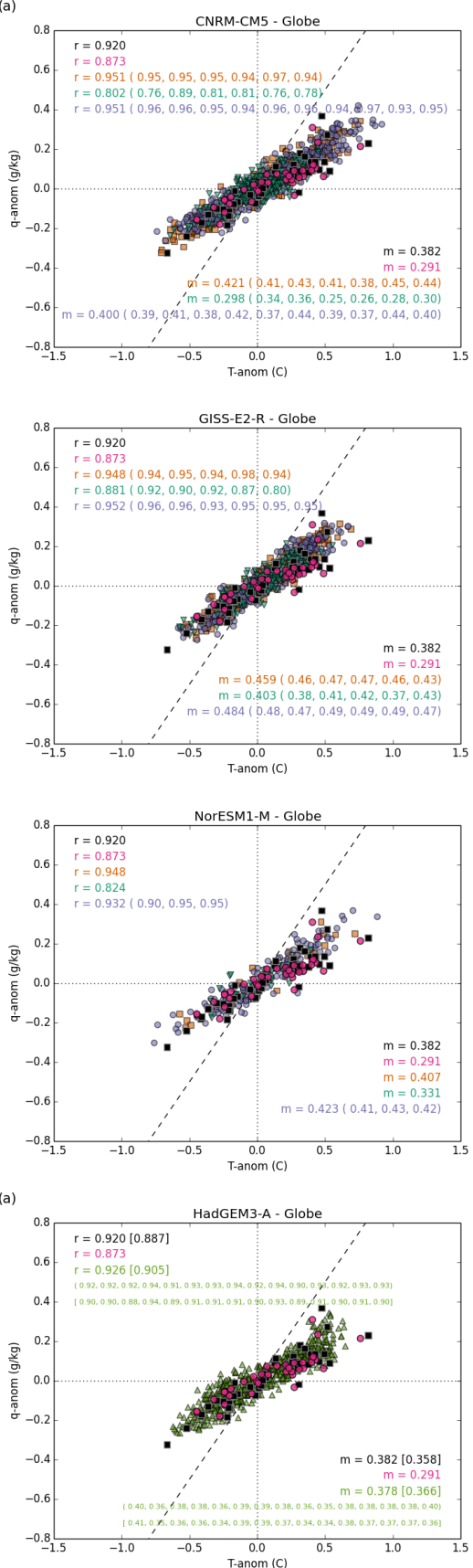

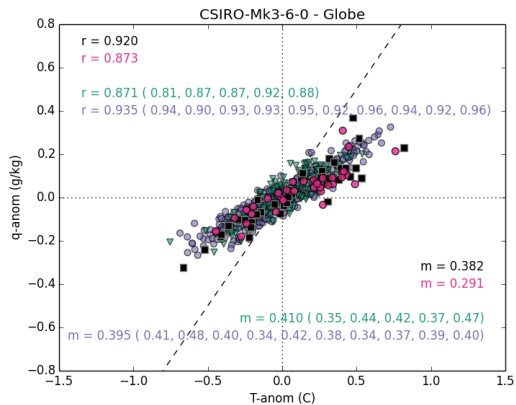

(g)
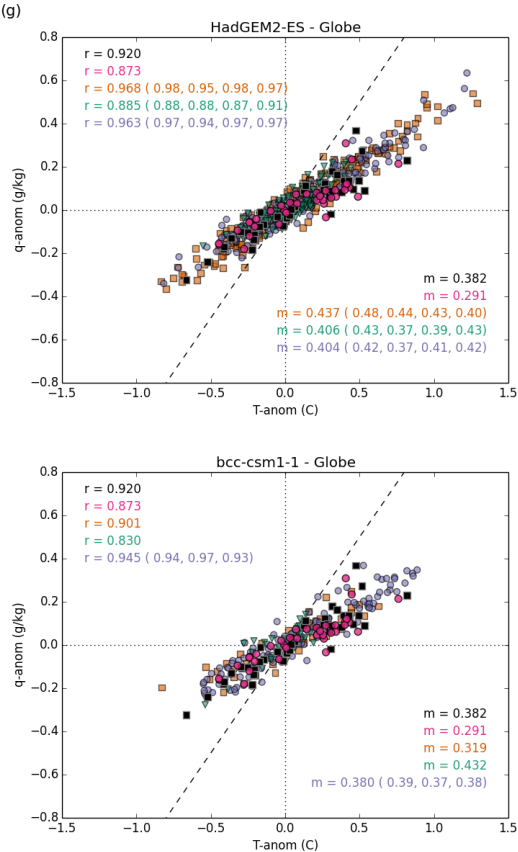

Figure S33. Relationships in global average temperature and specific humidity for CMIP5 models compared to HadISDH and ERA-Interim. Correlations (r) are shown in the top left hand corner. The gradient of the line of best fit is given in the bottom right hand corner. HadISDH is shown in black. ERA-Interim is shown in magenta. Historical, historicalNAT and historicalGHG are shown in purple, green and orange respectively. The ensemble mean value is given for each model with individual member values in parentheses. Values in square brackets are calculated using data matched to the coverage of ERA-Interim, for both HadISDH and HadGEM3-A. 

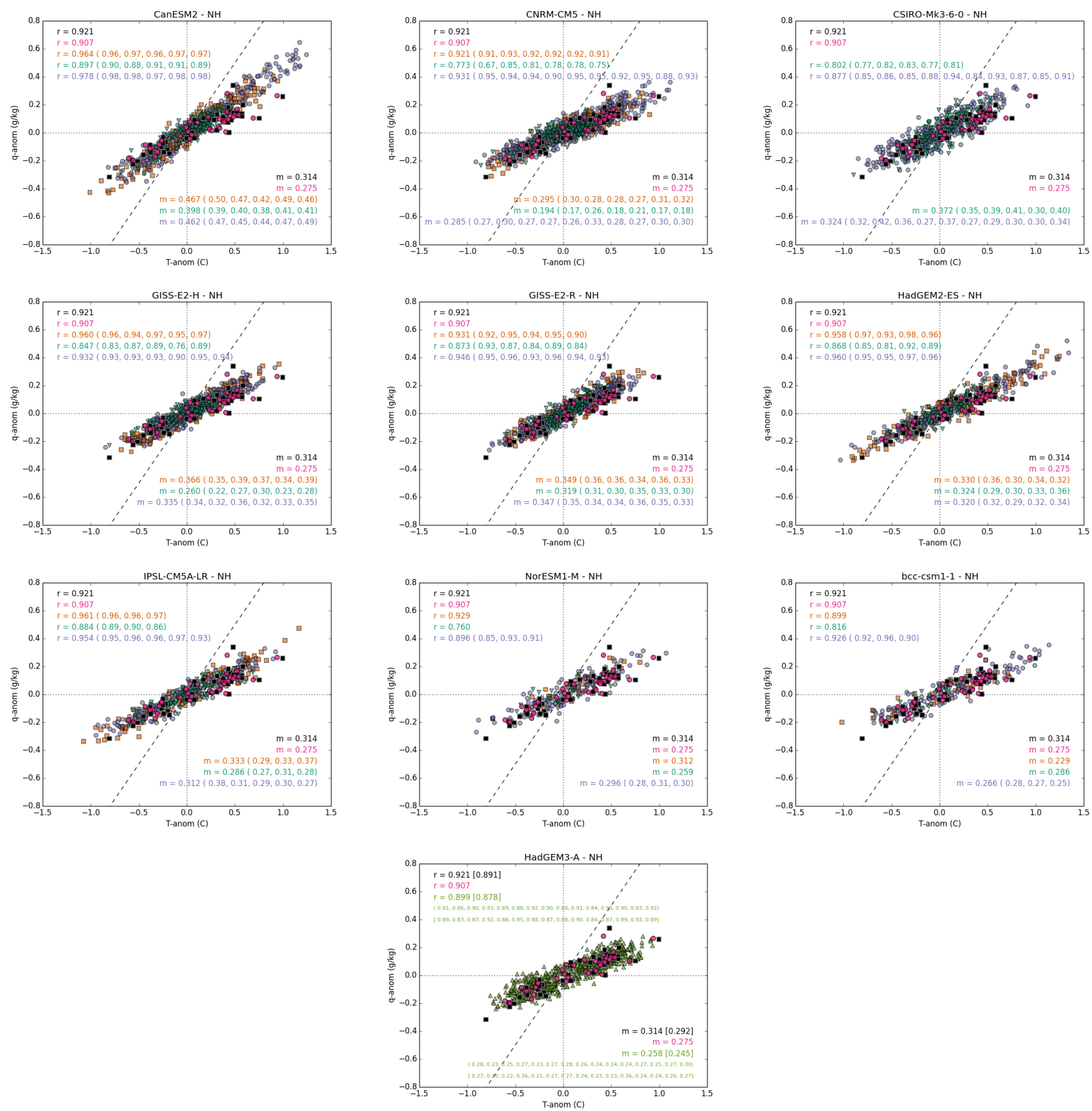

Figure S34. Relationships in Northern Hemisphere average temperature and specific humidity for CMIP5 models compared to HadISDH and ERA-Interim. Correlations (r) are shown in the top left hand corner. The gradient of the line of best fit is given in the bottom right hand corner. HadISDH is shown in black. ERA-Interim is shown in magenta. Historical, historicalNAT and historicalGHG are shown in purple, green and orange respectively. The ensemble mean value is given for each model with individual member values in parentheses. Values in square brackets are calculated using data matched to the coverage of ERA-Interim, for both HadISDH and HadGEM3-A. 

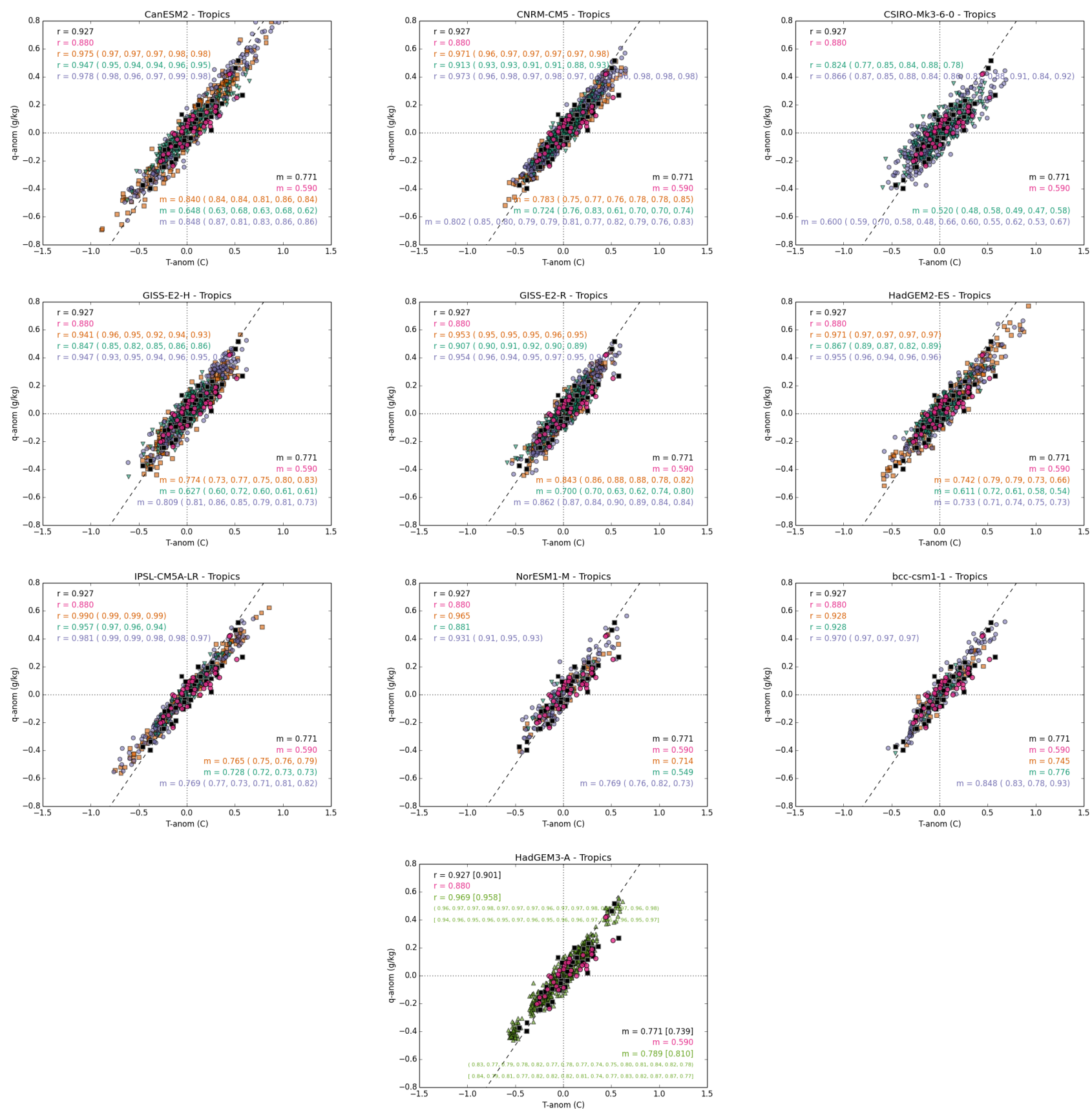

Figure S35. Relationships in tropical average temperature and specific humidity for CMIP5 models compared to HadISDH and ERAInterim. Correlations (r) are shown in the top left hand corner. The gradient of the line of best fit is given in the bottom right hand corner. HadISDH is shown in black. ERA-Interim is shown in magenta. Historical, historicalNAT and historicalGHG are shown in purple, green and orange respectively. The ensemble mean value is given for each model with individual member values in parentheses. Values in square brackets are calculated using data matched to the coverage of ERA-Interim, for both HadISDH and HadGEM3-A. 


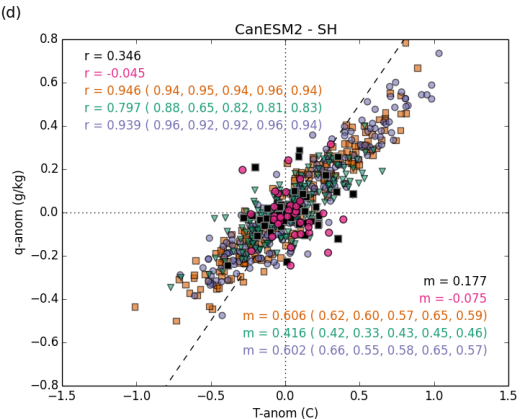

(f)
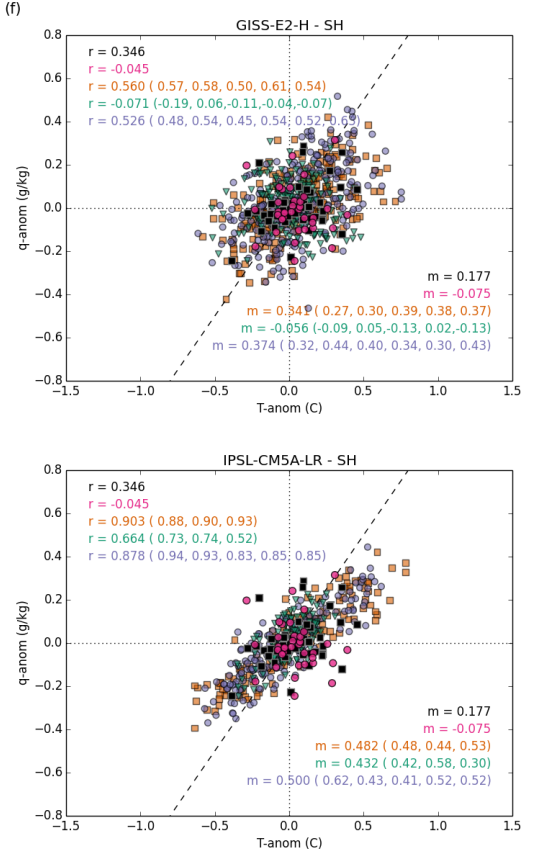

(b)
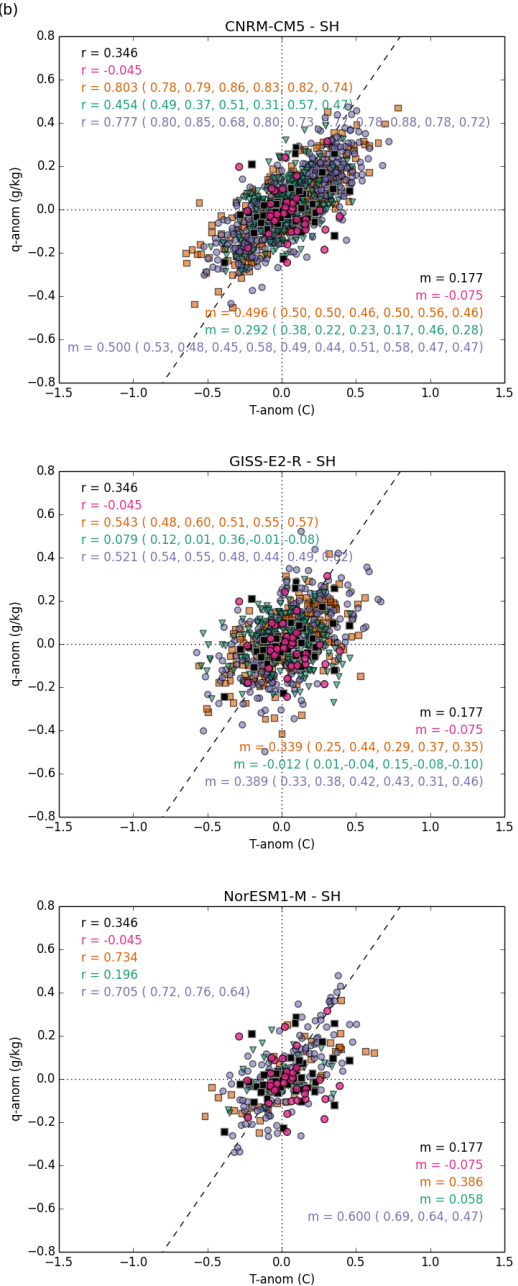

(c)

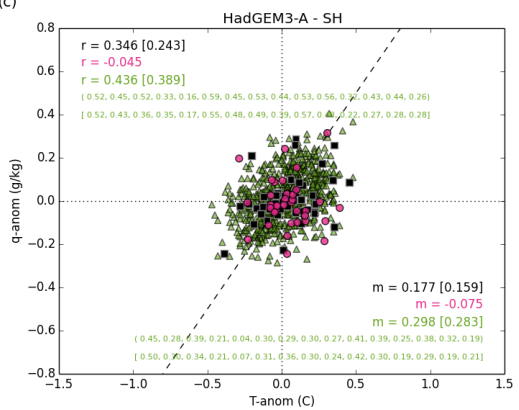

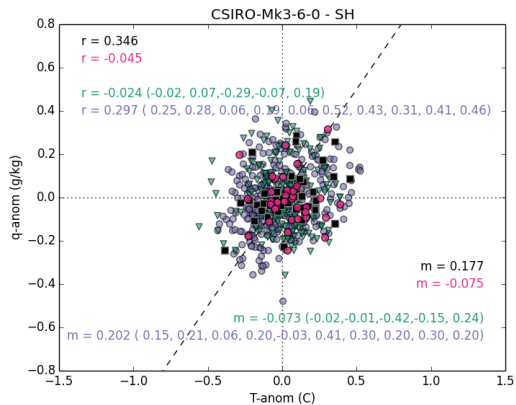

(h)
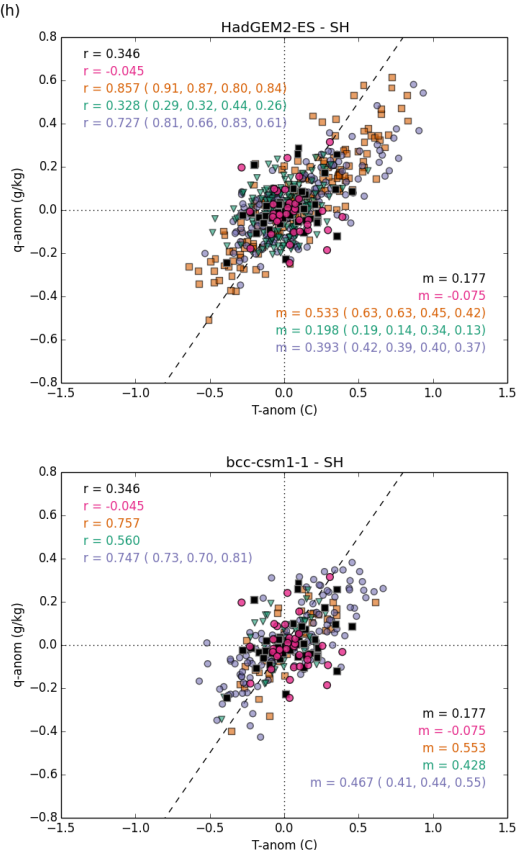

Figure S36. Relationships in Southern Hemisphere average temperature and specific humidity for CMIP5 models compared to HadISDH and ERA-Interim. Correlations (r) are shown in the top left hand corner. The gradient of the line of best fit is given in the bottom right hand corner. HadISDH is shown in black. ERA-Interim is shown in magenta. Historical, historicalNAT and historicalGHG are shown in purple, green and orange respectively. The ensemble mean value is given for each model with individual member values in parentheses. Values in square brackets are calculated using data matched to the coverage of ERA-Interim, for both HadISDH and HadGEM3-A. 


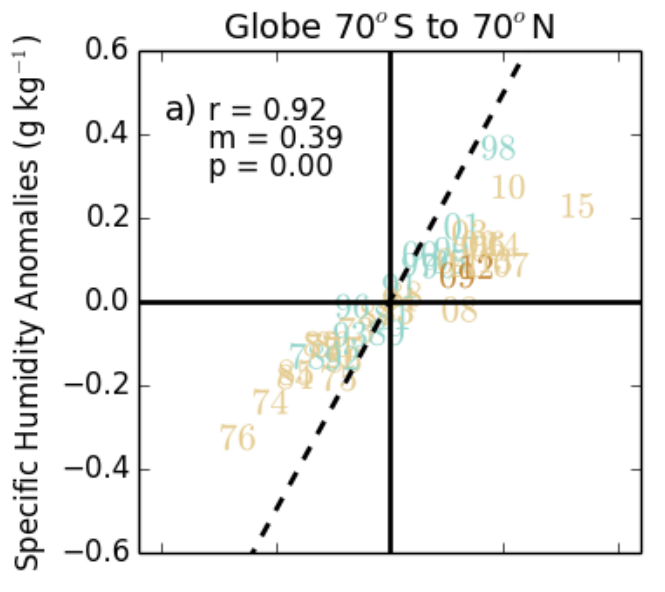

N. Hemisphere $20^{\circ} \mathrm{N}$ to $70^{\circ} \mathrm{N}$
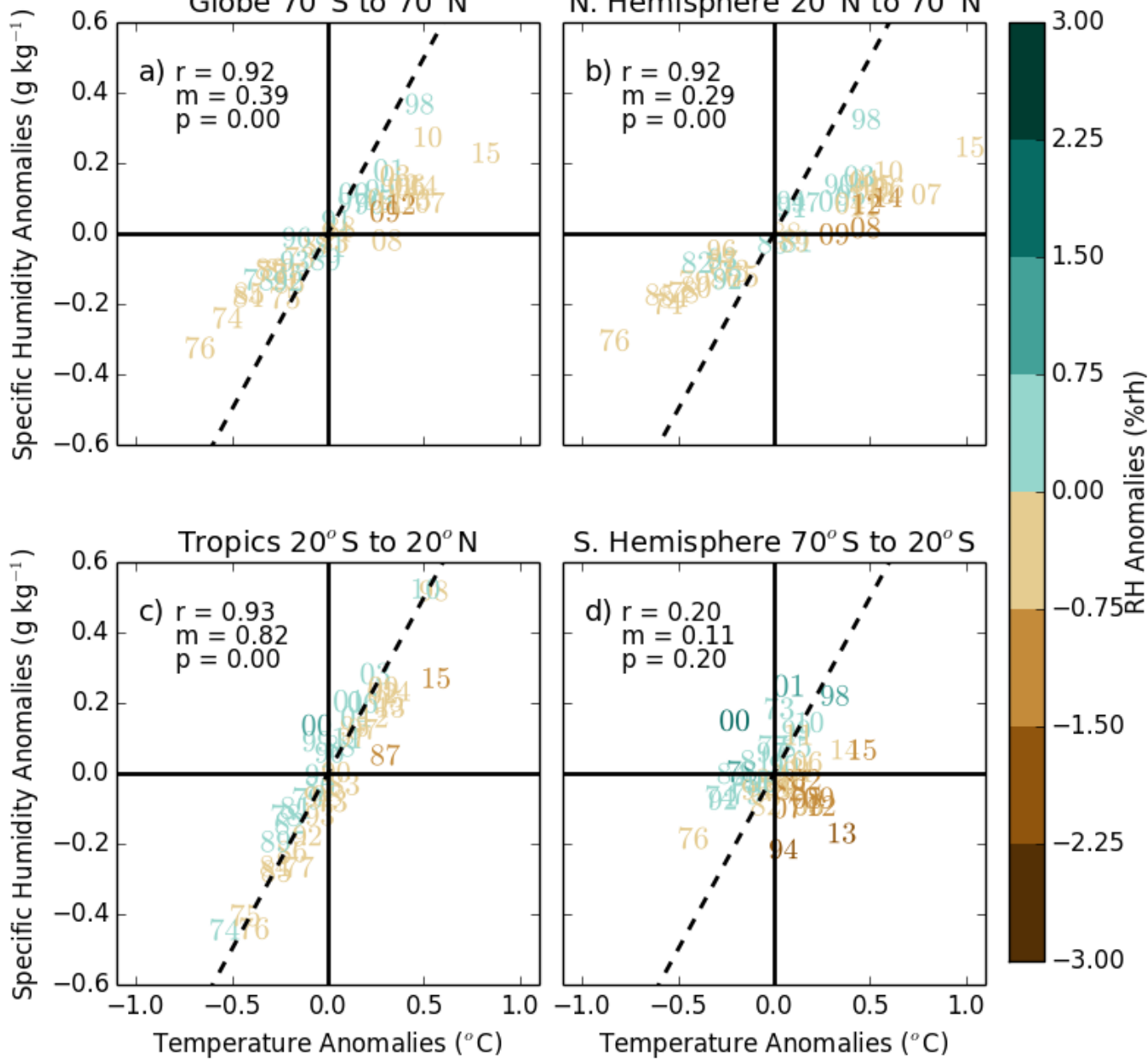

Figure S37. Temperature-specific humidity relationship, with the relative humidity anomaly as the colour scale, with the years as the points. The four zonal regions used in this analysis are shown in the four panels. 